\title{
Experimental study of lipid membranes: lipid domain formation and peptide aggregation
}

\author{
Dissertation \\ zur Erlangung des Doktorgrades \\ der Mathematisch-Naturwissenschaftlichen Fakultäten \\ der Georg-August-Universität zu Göttingen
}

\author{
Vorgelegt von \\ Vitaliy Oliynyk \\ aus Nizhin, Ukraine
}

Göttingen 2005 
D 7

Referent: Prof. Dirk Ronneberger

Korreferent: Prof. Thomas Heimburg Tag der mündlichen Prüfung: 


\section{Acknowledgements}

I pay special thanks to my supervisors Prof. Thomas Heimburg and Dr. Udo Kaatze for their versatile support, also in difficult periods, and for inspiring discussions. They also suggested many interesting points and perspectives to me and provided me with a reasonable degree of freedom in my work. Furthermore they skilfully revised this thesis. I am also thankful to Prof. Dirk Ronneberger who kindly agreed to be a referent of my thesis.

I am very grateful to Dr. Tilman Schäffer who introduced me at the beginning of my work into the basics of operating AFM. From him I learnt a lot about interpreting of AFM images, essential trick and skills. I also thank to Prof. Thomas Bjørnholm for a possibility to work with their equipment at the Nano-Science Center of University of Copenhagen. I am thankful to Dr. Manfred Konrad from MPI for biophysical chemistry in Göttingen who supplied us with peptides synthesized in his group.

Many thanks to Heiko Seeger for his efforts in revising of this thesis and for valueable discussions of results. I am also grateful to Irek Iwanowski and to Heiko Seeger for their wonderful sense of humour to lighten the work. I would just like to say many thanks to all co-workers at Membrane Thermodynamics Group and at Complex Fluids Group for a good time working with them, all their help and co-operations.

I gratefully acknowledge my family for their support, especially to my wife Alona who shares with me all difficulties and helps to overcome them. 



\title{
Abbreviations
}

\author{
AFM: Atomic Force Microscopy \\ DSC: Differantial Scanning Calorimetry \\ CFM: Confocal Fluorescence Microscopy \\ MC: Monte Carlo simulation \\ MLV: Multilamellar Vesicle \\ GUV: Giant Unilamellar Vesicle \\ LUV: Large Unilamellar Vesicle \\ SUV: Small Unilamellar Vesicle \\ DLPC: 1,2-dilauroyl-sn-glycero-3-phosphocholine \\ DMPC: 1,2-dimyristoyl-sn-glycero-3-phosphocholine \\ DPPC: 1,2-dipalmitoyl-sn-glycero-3-phosphocholine \\ DSPC: 1,2-distearoyl-sn-glycero-3-phosphocholine
}





\section{Contents}

$\begin{array}{ll}\text { Contents } & 7\end{array}$

$\begin{array}{llr}1 & \text { Introduction } & 9\end{array}$

2 Materials and methods $\quad 37$

2.1 Experimental methods . . . . . . . . . . . . . . 37

2.1.1 Atomic force microscopy . . . . . . . . . . . . . 37

2.1.2 Differential scanning calorimetry . . . . . . . . 44

2.1.3 Ultrasonic spectrometry . . . . . . . . . . . . . 47

2.2 Materials and sample preparation . . . . . . . . . . . . . 49

2.2.1 Substances: lipids and peptides . . . . . . . . . . . 49

2.2.2 Preparation of multilamellar vesicle suspensions . . . . 53

2.2.3 Sample preparation for AFM experiments . . . . . . 53

2.2.4 Sample preparation for DSC and ultrasonic spectrometry experiments . . . . . . . . . . . 55

$\begin{array}{lll}3 & \text { Results } & 57\end{array}$

3.1 Supported lipid membranes . . . . . . . . . . . 57

3.2 Domain formation in two-component lipid membranes. . . . . 62

3.3 Peptide-containing lipid membranes . . . . . . . . . . . . 65

3.3.1 DSC experiments . . . . . . . . . . . . . 65

3.3.2 Ultrasonic sound velociy experiments . . . . . . . . 67

3.3.3 AFM experiments . . . . . . . . . . . 70 
4 Discussion and conclusions 79

Bibliography 87

List of Figures 97 


\section{Chapter 1}

\section{Introduction}

A biological cell (see Figure 1.1) is highly organized with many functional units or organelles. Most of these units are limited by one or more membranes that give rise to a variety of physiologically important functions. Cell mem-

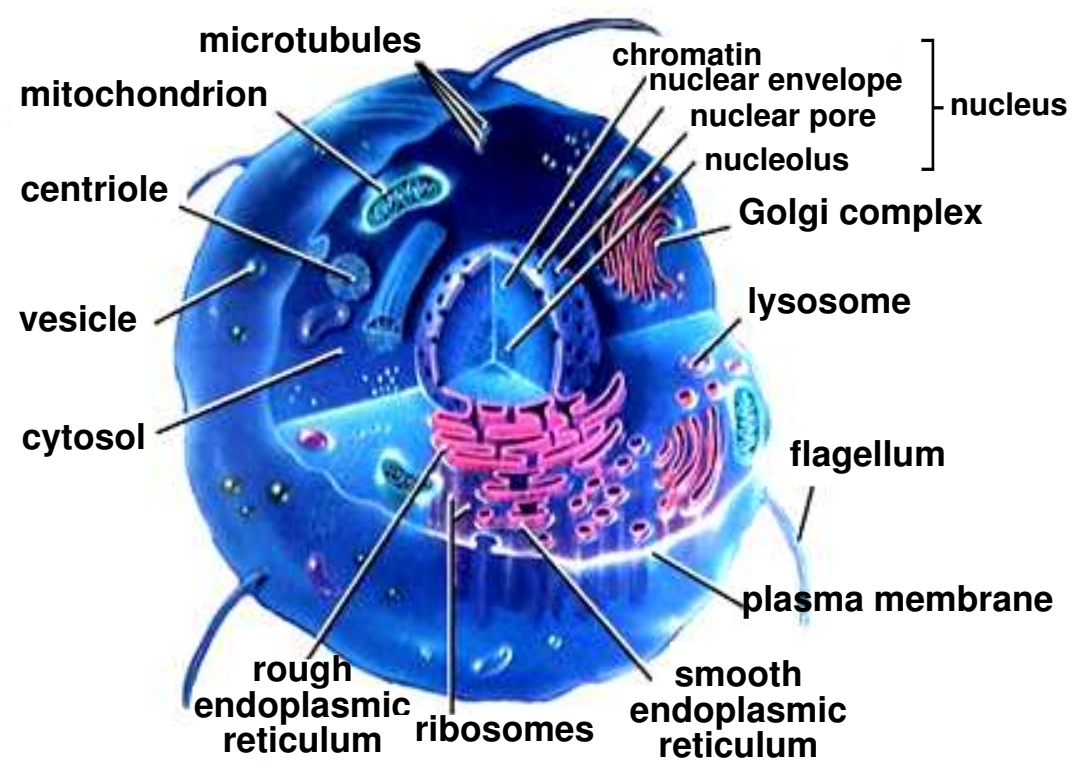

Figure 1.1: Schematic illustration of an animal cell ${ }^{1}$.

branes are crucial to the functioning of the cell. The plasma membrane

\footnotetext{
${ }^{1}$ Adapted from http://www.cbv.ns.ca/bec/science/cell/.
} 


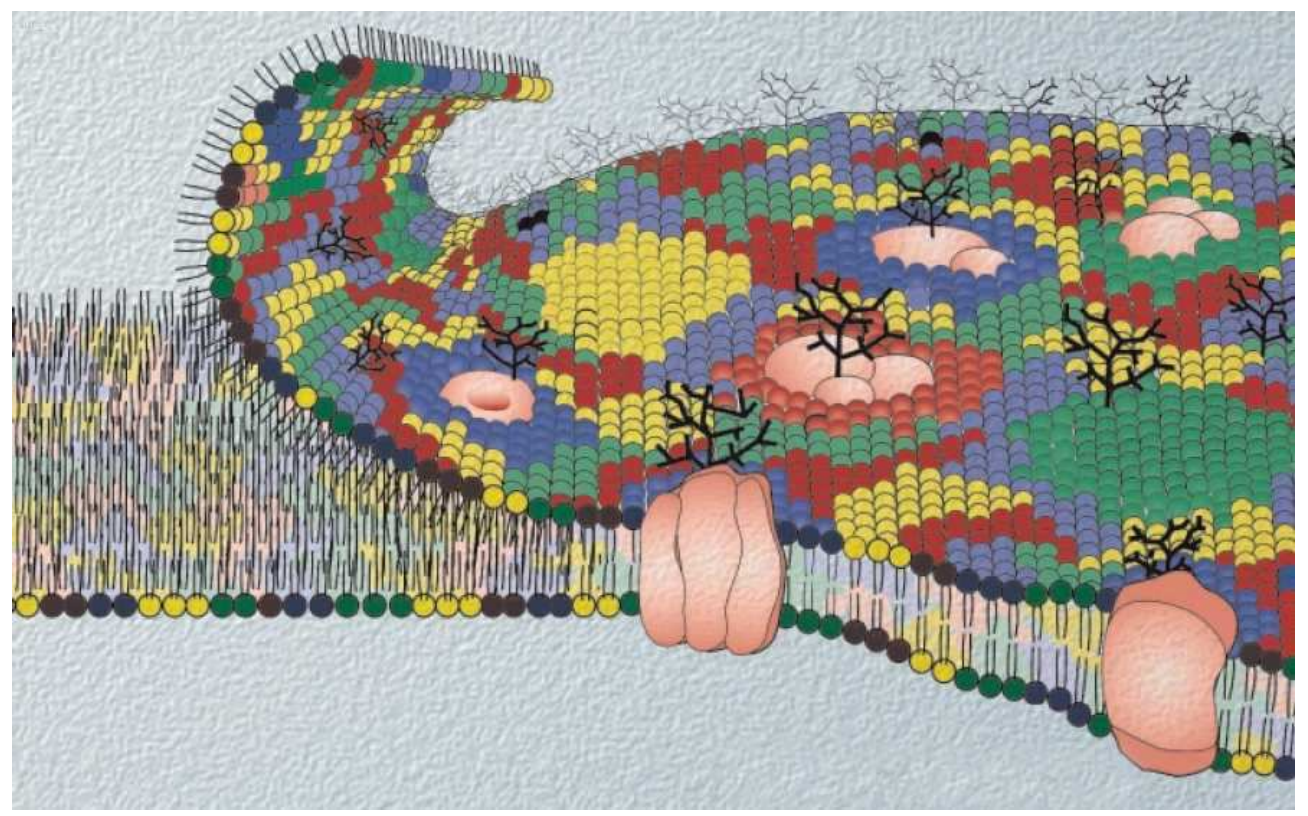

Figure 1.2: Schematic illustration of the plasma membrane of a cell. The lipid bilayer is the main structural element in which proteins (pink) are embedded. Lipid headgroups are depicted in different colors which illustrate the important point that the membrane consists of a number of different lipids and they are not necessarily homogeneously distributed in the lipid bilayer ${ }^{2}$.

encloses the cell, defines its boundaries, and maintains the essential differences between the cytosol and the extracellular environment. Inside the cell the membranes of the endoplasmic reticulum, Golgi apparatus, mitochondria, and other membrane-bounded organelles in eukariotyc cells maintain the characteristic differences between the contents of each organelle and the cytosol. Ion gradients across membranes, established by activities of specialized membrane proteins, can be used to synthesize ATP, to drive the transmembrane movement of selected solutes, or, in nerve and muscule cells, to produce and transmit electrical signals. In all cells the plasma membrane also contains proteins that act as sensors of external signals, allowing the cell to change its behavior in response to environmental cues (Alberts et al., 1994).

\footnotetext{
${ }^{2}$ Adapted from Edidin (2003).
} 


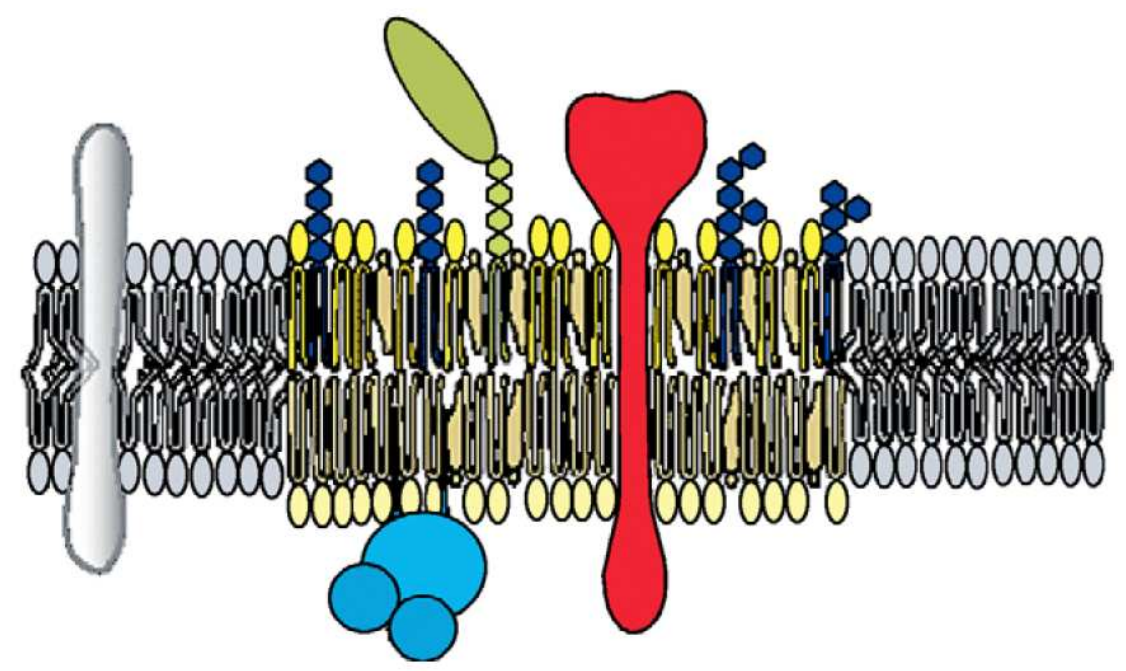

Figure 1.3: Schematic illustration of a membrane raft, consisting of a lipid patch enriched in sphingolipids, glycolipids, and cholesterol to which certain proteins are attached $^{3}$.

The main molecular constituents of biological membranes are lipids and proteins, which are organized into a bilayer of lipid molecules with integral proteins embedded and peripheral proteins attached. A schematic illustration of this biomembrane architecture is shown in Figure 1.2. Membrane associated proteins are involved in a great number of biochemical reactions making the membrane an important reaction site in the cell.

The fluid mosaic model by Singer \& Nicholson is still the textbook picture of cell membrane organization. It proposes that the lipid bilayer functions as a neutral two-dimensional solvent in which proteins and lipids freely diffuse in the plane of the membrane (Singer and Nicolson, 1972). Currently, however, membranes are viewed as a mosaic of different compartments or domains maintained by an active cytoskeleton network (Simons and Ikonen, 1997; Jacobson and Dietrich, 1999). Due to interactions between components, several types of subdomains can form with different characteristics and functions. Lipids are likely to play an important role in the formation of

\footnotetext{
${ }^{3}$ Adapted from www.glycoforum.gr.jp/science/word/ glycolipid/GLD01E.html.
} 


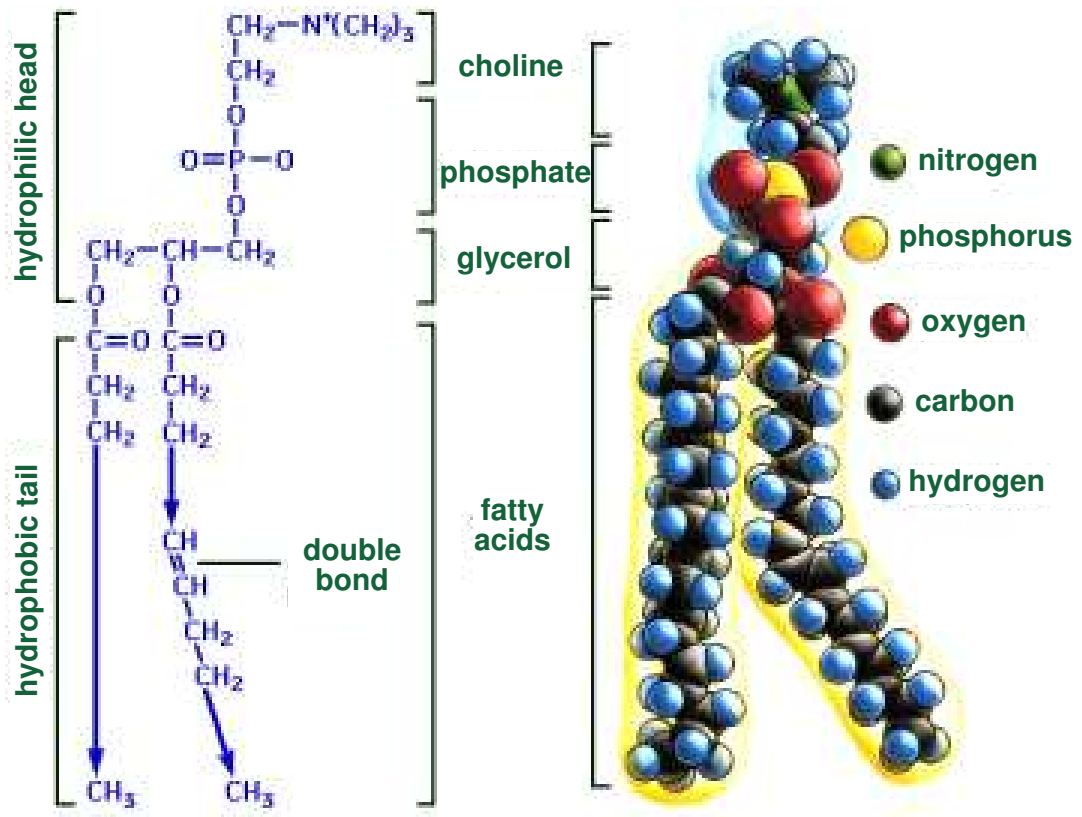

Figure 1.4: The example schema of a phospholipid ${ }^{4}$.

so-called lipid-enriched microdomains or lipid rafts (see Figure 1.3), adding another order of complexity to the membrane model (Thompson and Tillack, 1985; Jacobson and Dietrich, 1999). Rafts within the plasma membrane of eukariotyc cells have been implicated in many important cellular processes, such as polarized sorting of apical membrane proteins in epithelial cells (Simons and van Meer, 1988), dendritic proteins in neurons (Dotti et al., 1991) and signal transduction (Stauffer and Meyer, 1997; Kurzchalia and Parton, 1999).

Biomembranes are highly complex mixtures consisting of a vast number of proteins and as many as 1000 different lipids (Alberts et al., 1994; Dowhan, 1997). This complexity makes it virtually impossible to make detailed physical conclusions from studies of native membranes and a simplification is therefore required. Lipid bilayers containing just a single lipid or a few different lipids are popular model systems for studies of the physical principles of biological membranes.

\footnotetext{
${ }^{4}$ Adapted from http : //www.agen.ufl.edu/ chyn/age2062/lect/lect_06.
} 
There is no strict definition of the term lipid that is generally accepted (Larsson, 1994). A good definition was recently proposed (Christie, 1987): "Lipids are fatty acids and their derivatives, and substances related biosynthetically or functionally to these compounds". Lipids have a variety of biological roles: they serve as fuel molecules, highly concentrated energy stores, signal molecules, and components of membranes. Here, our focus is on lipids as membrane constituents.

The lipid composition varies between cells and organelles (see Table 1.1). The three major kinds of membrane lipids are phospholipids, glycolipids, and cholesterol. The most abundant are the phospholipids. The platform on which phospholipids are built may be glycerol, a 3-carbon alcohol, or sphingosine, or a more complex alcohol (Berg et al., 2002). All of the lipid molecules in the plasma membrane are amphiphilic (or amphipathic) - that is, they have a hydrophilic ("water-loving", or polar) end and a hydrophobic ("water-hating", or nonpolar) end. Phospholipids have a polar headgroup and two hydrocarbon tails (see Figure 1.4). The tails are usually fatty acids, and they can differ in length (they normally contain between 14 and 24 carbon atoms). One tail usually has one or more cis-double bonds (that is, it is unsaturated), while the other tail does not (that is, it is saturated). As indicated in Figure 1.4, each double bond creates a small kink in the tail. Differences in the length and saturation of the fatty acid tails are important because they influence the ability of phospholipid molecules to pack against one another, and for this reason they affect the fluidity of the membrane. The amphiphilic nature of lipid molecules defines their ability to form different aggregates in water or water solutions, among which a lipid bilayer is the most relevant structure for biological membranes.

Mixtures of lipids and water are polymorphic. In an aqueous environment lipids self-assemble spontaneously in order to protect their hydrophobic tails from contact with the water molecules. The macroscopic structure of these molecular aggregates depends on the chemical structure of the lipid as well as on the water content. Even for single purified lipids there is more than 


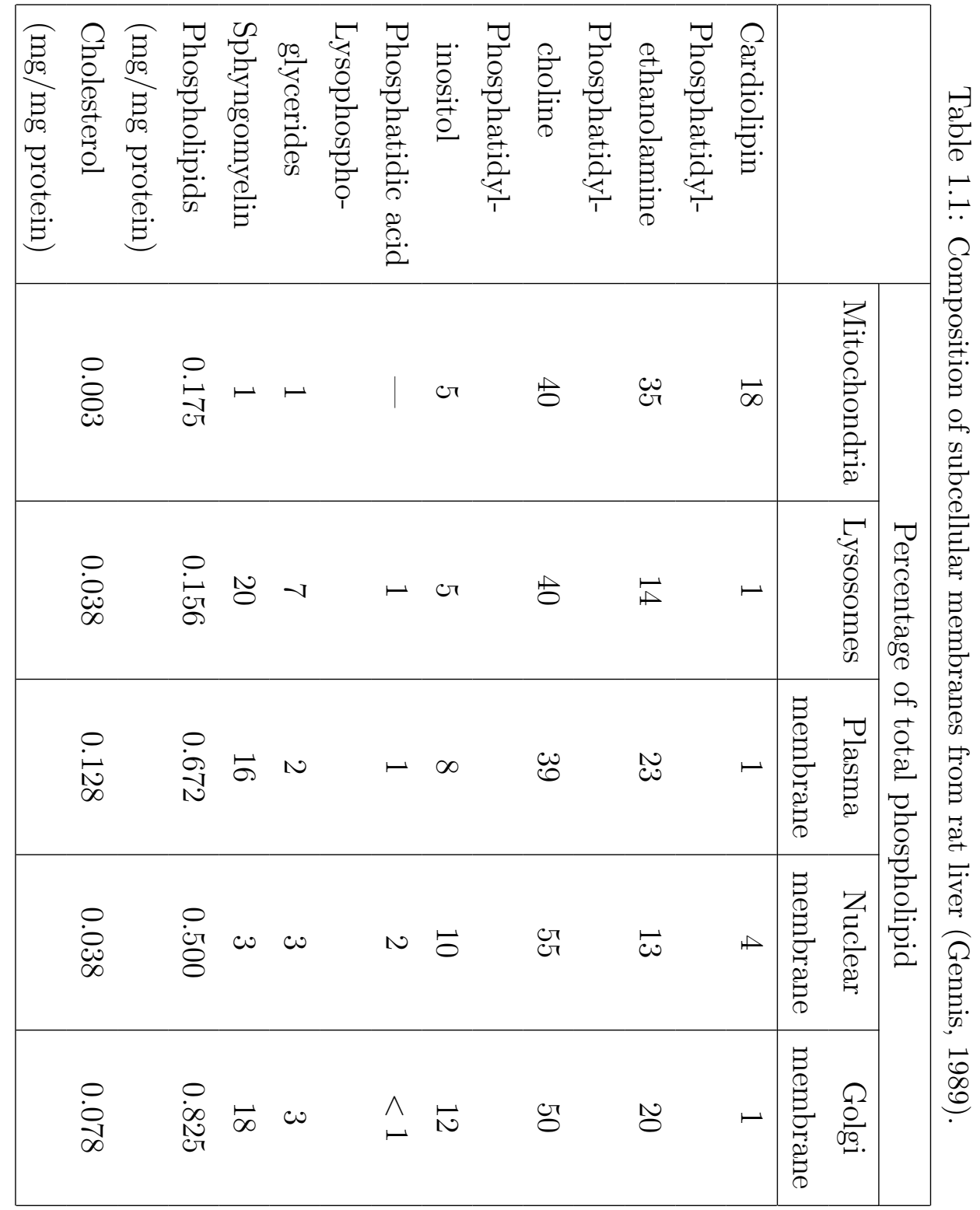


one kind of organized structure when hydrated. The particular form which predominates depends on such parameters as the lipid concentration, temperature, pressure, ionic strength, and pH (Gennis, 1989). Some of the variety of such molecular assemblies are shown in Figure 1.5.

The lipid aggregates are stabilized primarily by the hydrophobic interaction resulting from the inability of lipid hydrocarbon tails to hydrogen bond with water. At the interface between hydrocarbon tail and water, there is a higher energy state for the water because hydrogen bonds are lost. It is energetically favorable for the hydrocarbon tail to associate with other hy-

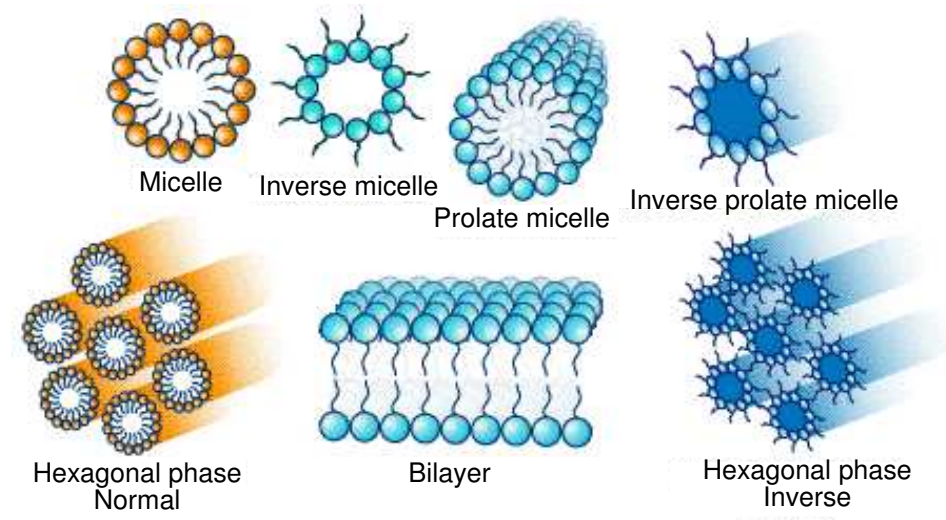

Figure 1.5: Examples of lipid structures in water solutions ${ }^{5}$.

drocarbon and to minimize the surface area of contact with water. Lipids can stabilize their hydrocarbon tails in water by covering the hydrocarbon surface with their hydrophilic headgroups while their hydrocarbon regions associate with hydrocarbons (see Figure 1.5). In terms of free energy, the mixing of hydrocarbon tails and water causes a decrease in entropy (another way of saying this is to state that the entropy of water is decreased at the interface between hydrocarbon and water). Other stabilizing forces are van der Waal's interactions between the hydrocarbon chains, electrostatic interactions and hydrogen bonds between the polar heads.

\footnotetext{
${ }^{5}$ Adapted from http://bioteach.ubc.ca/Bio-industry/Inex/.

${ }^{6}$ Adapted from http://courses.cm.utexas.edu/jrobertus/ch339k/overheads-2.htm.
} 


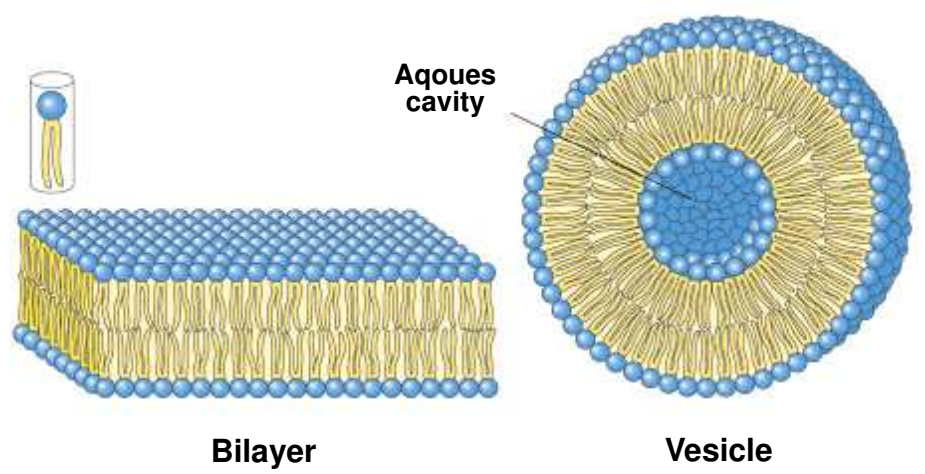

Figure 1.6: Lipid bilayer and lipid vesicle ${ }^{6}$.

In relation to biological systems the most important structure is the lipid bilayer which is called very often lipid bilayer membrane. The crucial physical characteristic of the membrane phospholipids and other double-chain amphiphilic lipids is its propensity to form bilayers in aqueous dispersions, in the form of single vesicles or multilamellar structures (Cantor, 1999). These are schematically illustrated in Figure 1.6, which shows that the lipids arrange themselves with polar headgroups exposed to the aqueous phase and the hydrocarbon tails clumped together generating their own non-aqueous environment. The bilayer acts as a membrane, separating components encapsulated on the inside of the vesicle from those on the outside. Clearly the thickness of the bilayer will depend on the length and stiffness of the hydrocarbon tails of the lipids.

Bilayers composed of a single lipid species display an order-disorder transition (the so-called melting transition) at well defined temperatures. The low temperature lipid state has hydrocarbon chains predominantly ordered in an all-trans configuration and is for historical reasons called the "gel" state. The high temperature state with unordered chains is called the "fluid" state. This transition is due to the following: There is free rotation about each $\mathrm{C}-\mathrm{C}$ bond in the hydrocarbon chains with preferred energy minima at given angles - one being the absolute energy minimum (trans-conformation) and the other two having equal excitation energies $\left(\right.$ gauche ${ }^{+}$and gauche ${ }^{-}$confor- 


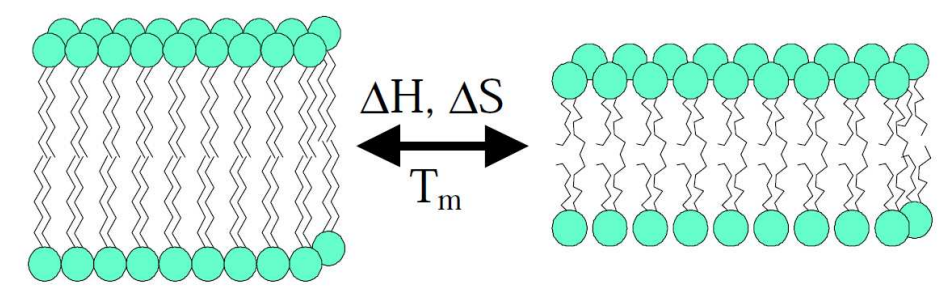

Figure 1.7: Scheme of the melting transition in a lipid bilayer ${ }^{7}$.

mations). When all the C-C bonds are in trans-conformation (all-trans), the two chains are parallel and are maximally extended. However, the probability for the occurrence of a gauche-bond increases with increasing temperature. At high temperatures, the chains are not correlated and are fully disordered. Figure 1.7 illustrates the bilayer melting transition.

The chain-melting (or gel-to-fluid) transition is an endothermic process with a relatively large heat $(8700 \mathrm{cal} / \mathrm{mol})^{8}$. The entropy changes by about $26 \mathrm{cal} /(\mathrm{mol} \cdot \mathrm{K})^{8}$, the membrane volume increases $(4 \%)^{8}$ and the bilayer thickness decreases $(-16 \%)^{8}$. The heat capacity is defined as the amount of heat required to raise the temperature of the system by a given temperature increment $\left(c_{P}=\frac{\Delta Q}{\Delta T}\right)$. A typical heat capacity profile of a lipid system undergoing melting transition is given in Figure 1.8. It is evident that the transition half-width is very small (about 0.1 degree). Supposing that each lipid in the system melts independently, the calorimetric heat in a two-state transition is equal to the van't Hoff heat, and the transition half-width should be about 60 degrees (Gennis, 1989). This is the reason for calling the melting transition cooperative. The cooperativity implies that the lipid molecules do not undergo a melting transition independently but in a correlated manner. However, the lipid melting cannot generally be classified as a first-order phase transition. All thermodynamic properties, like membrane area, volume and thickness, vary in a continuous though dramatic fashion during the transition (Mouritsen, 1991a). This has led to the proposal that the lipid

\footnotetext{
${ }^{7}$ Adapted from Ivanova (2000).

${ }^{8}$ Data for DPPC, from Heimburg (1998).
} 


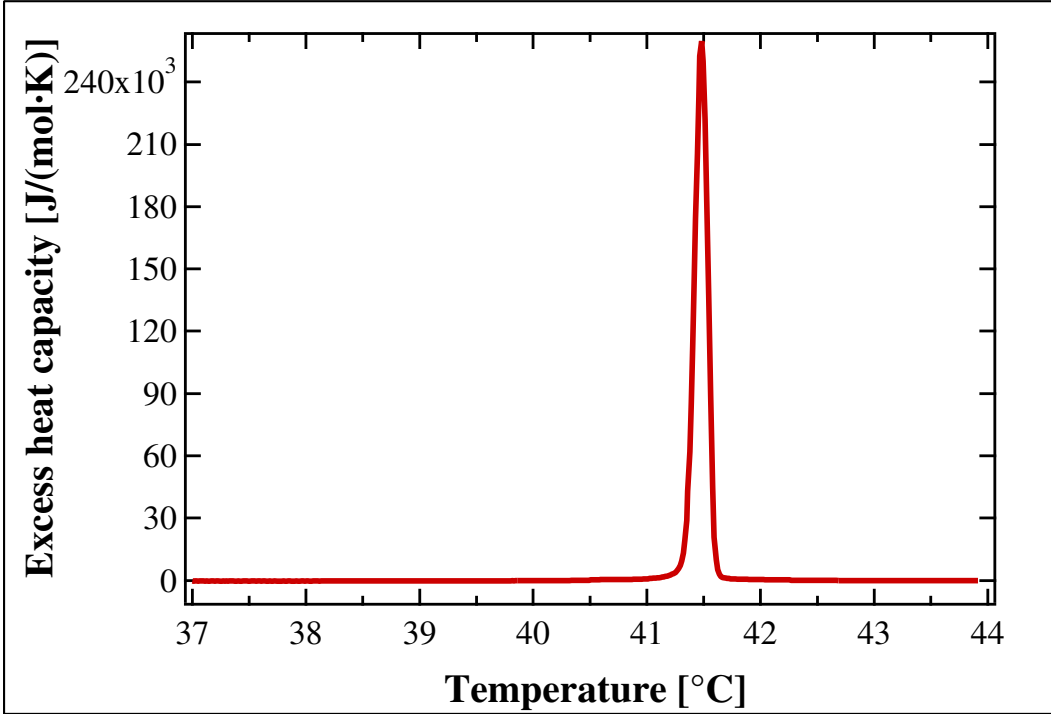

Figure 1.8: Heat capacity profile of a DPPC aqueous dispersion.

melting transition is pseudo-critical, i.e., in principle of first order but very close to a critical point and consequently strongly dominated by thermal fluctuations (Mouritsen, 1991a).

In recent years, it has become evident that the lateral organization of the lipid bilayer is far from random or homogeneous, but rather structured in terms of lipid domains. The range of length scales over which these domains persist varies from nanometers up to the size of the whole cell. Results from both experimental and theoretical membrane studies have clearly demonstrated that the formation of lipid domains and small-scale lipid structures, in certain contexts called rafts, play an important role as regulating units for a large number of biological functions that take place in or are associated with lipid membranes (Simons and Ikonen, 1997; Brown and London, 1998). Large-scale lipid structures can be stabilized by static thermodynamic phase separation, by coupling via integral membrane proteins to the cytoskeleton, or by locally curved membrane regions (Sackmann, 1994; Kusumi and Sako, 1996). Small-scale lipid domain formation is likely to be controlled by the nature of the lipid-lipid interactions (Nielsen et al., 2000). It has been shown 
$\mathbf{a}$

b
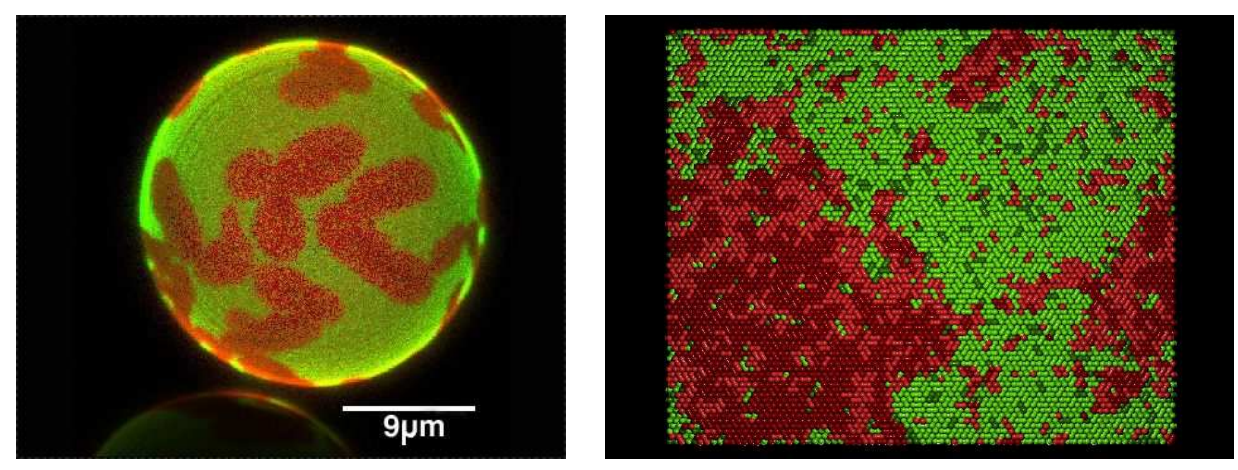

Figure 1.9: (a) Confocal microscopy image of a 33:67 DLPC:DPPC mixture at $26^{\circ} \mathrm{C}$, showing domain formation (gel domains in red, fluid domains in green). (b): Representative Monte Carlo snapshot of a 70:30 DMPC:DSPC mixture at $28{ }^{\circ} \mathrm{C}$. Red domains correspond to gel lipids, green domains to fluid lipids. Note the different length scales of the domains: macroscopic and microscopic domains. Adapted from Seeger et al. (2005); Hac et al. (2005).

that lipid membrane heterogeneity and the formation of micro-domains are of importance for the activity of membrane-associated enzymes and proteins. Specific examples of the close interplay between lipid membrane microstructure and function include the binding and activation of peripheral enzymes and proteins such as phospholipase A2, cytochrome C, and kinase C (Hønger et al., 1996; Kinnunen, 1996; Yang and Glaser, 1996).

Binary lipid mixtures are highly simplified models of native membranes, which consist of a big number of different lipids, but already those simple systems can be used for an achievement of basic understanding of lipid domains coexisting. Recent studies of two-component phosphocholine lipid membranes, which were done in our lab (Seeger et al., 2005; Hac et al., 2005), have revealed the lipid phase separation - coexisting of fluid and gel lipid domains. In Figure 1.9a the confocal fluorescence microscopy image of a giant unilamellar vesicle on a coverslip are shown. This image was obtained from a DLPC:DPPC 33:67 mixture at room temperature. Red areas in the image 
correspond to lipid domain in gel phase and green areas correspond to fluid lipid domains. The formation of domains in this case can partially be related to the so called "hydrophobic mismatch", which depicts interactions of lipid chains with water when the hydrophobic chains differ in length. Hence, in order to protect its hydrophobic parts from the water molecules each lipid tends to surround itself with lipid chains of a similar length.

Monte Carlo simulations (MC) of DMPC:DSPC 70:30 mixtures at $28^{\circ} \mathrm{C}$ are presented in Figure 1.9b. The same color code as in the confocal microscopy image for gel and fluid domains has been used and darker and lighter tones of green and red colors in the MC snapshot depict the different sorts of lipids. As can be readily seen, the snapshot contains, small fluid domains within a gel matrix, or small gel domains embedded into a fluid matrix. A striking similarity of the domain shapes between simulation and experiment can be seen. Since the spatial resolution of the confocal microscope is limited by the wavelength of light (the length scale of the detected domain patterns is in the range of micrometers), it was not possible to explore in these experiments the microdomain organization of lipid macrodomains predicted by $\mathrm{MC}$ simulations .

The interactions between proteins and lipids are essential for membrane function and structure. Hydrophobic matching, i.e., the matching between the hydrophobic length of an integral membrane protein and the hydrophobic thickness of the lipid bilayer Figure 1.10a, can be an important factor in protein-lipid interactions (Mouritsen and Bloom, 1993; Jensen and Mouritsen, 2004). In 1984 Sackmann suggested (Sackmann, 1984) that the function of integral membrane proteins may be triggered by the lipid-bilayer properties as illustrated by the picture in Figure 1.10b. Already this picture anticipated the possibility that the local lipid environment around a membrane protein may have quite different properties from those that characterize the average, global state of the membrane, e.g., in terms of varying molecular composition, curvature, or hydrophobic thickness.

So far, little is known about the molecular interactions underlying the 
a
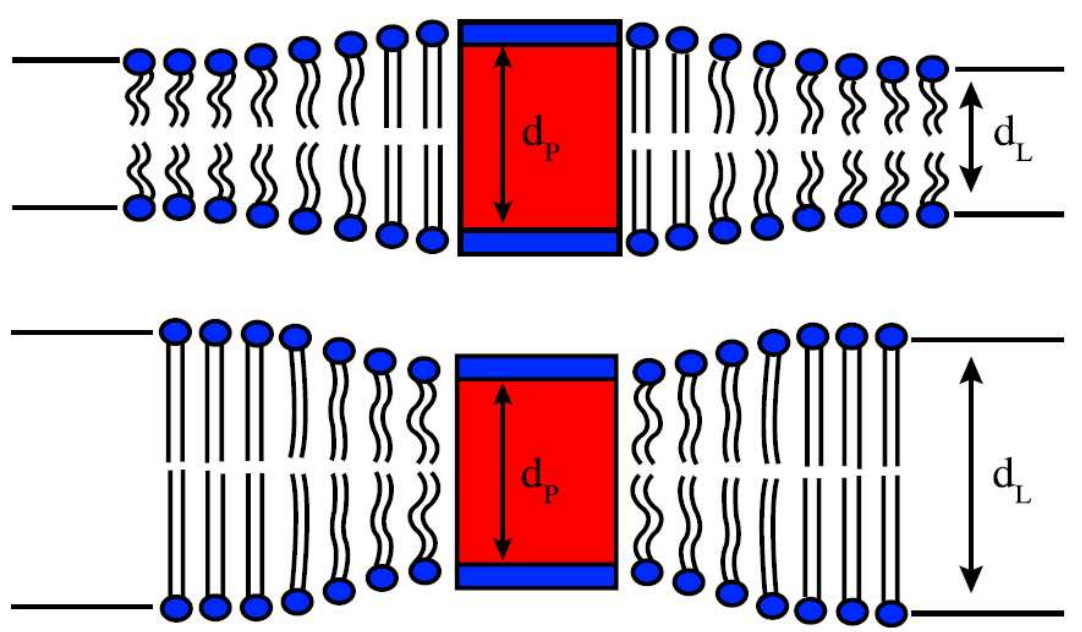

b

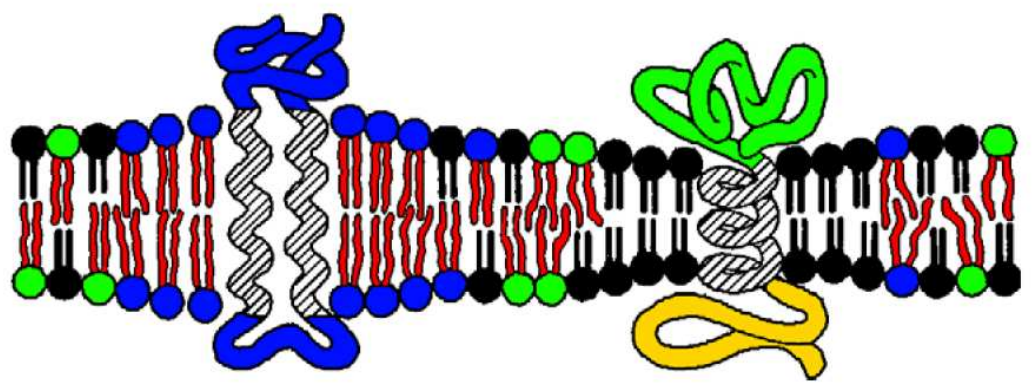

Figure 1.10: (a): Schematic illustration of hydrophobic matching of a integral membrane protein that is embedded in a thin (top) and a thick (bottom) membrane, $d_{L}$ is the lipid-bilayer hydrophobic thickness and $d_{P}$ is the protein hydrophobic length. Adapted from Dumas et al. (1999).(b): An illustration of triggering the function of an integral membrane protein by changing the hydrophobic mismatch. Adapted from Sackmann (1984).

effects of hydrophobic mismatch, which among other factors is due to the complexity of the systems studied. To get a better understanding of the lipid-protein interactions involved, one can use simple designed or native peptides as models for the membrane-spanning parts of integral membrane 
proteins.

Membrane-lytic or pore-forming peptides have been studied extensively in order to understand general aspects related to peptide-lipid interactions, as well as the relation of these interactions to the biological function of these peptides. The largest and most studied group of peptides includes short linear polypeptides ( $\geq 40$ amino acids). These polypeptides vary considerably in chain length, hydrophobicity and overall distribution of charge, but share a common $\alpha$-helical structure when associated with phospholipid membranes (Bechinger, 1997; Shai, 1999; Kourie and Shorthouse, 2000). Amphipathic, $\alpha$-helical peptides are abundant in nature, serving as membrane permeating agents in the host defence system of many organisms. Antibiotic peptides, such as alamethicin, isolated from Trichoderma viride fungus, the bee venom peptide melittin, the magainins of the African frog Xenopus laevis, and many others, are among the most intensively studied peptides (Cafiso, 1994; Dempsey, 1990; Zemel et al., 2003). Attempts to mimic nature and to design novel antibacterial drugs have stimulated numerous experimental and theoretical studies of amphipathic peptides.

A common feature found in most native membrane-lytic peptides is that the distribution and the amount of the net charge correlate with their biological function; peptides with a low net negative charge (e.g. N-hemolysin), or with a low net positive charge spread along their helix backbone (e.g. pardaxin, melittin) are mostly lytic to mammalian cells or to both mammalian and bacterial cells (Shai, 1999). On the other hand, native non-hemolytic antibacterial peptides contain high net positive charge contributed by a large number of basic amino acids which are distributed along the hydrophilic face of the amphipathic $\alpha$-helix. This feature has been proposed to account for their preferential activity against bacteria and not normal mammalian cells. Bacterial membrane is rich in acidic phospholipids. Therefore, the net positive charge of the antibacterial peptides facilitates their perturbing activity towards bacterial membrane. In contrast, the outer membrane of human erythrocytes (representatives of normal mammalian cells) is composed 

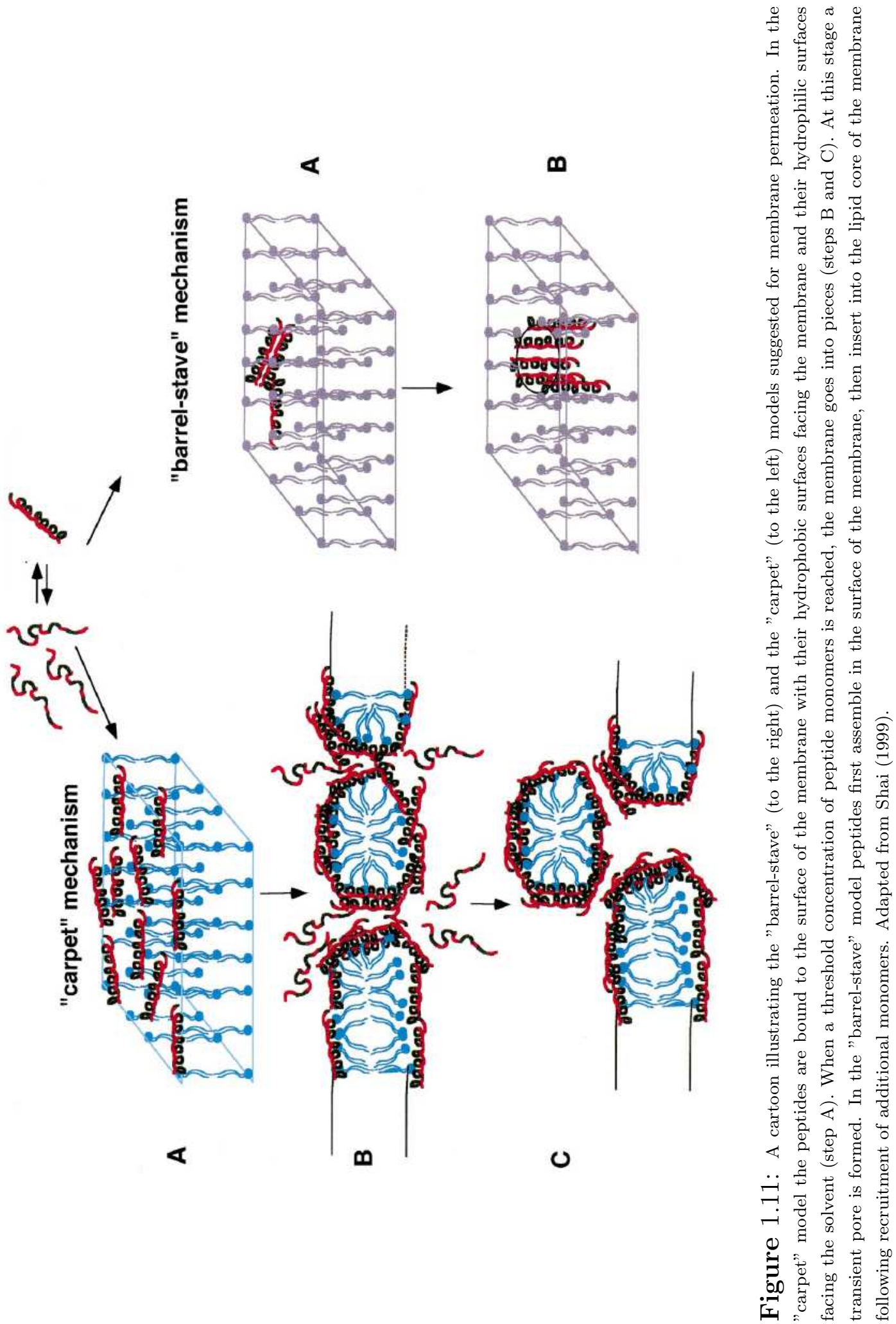
predominantly of zwitterionic phosphatidylcholine (PC) and sphingomyelin phospholipids (Verkleij et al., 1973).

Two alternative mechanisms were proposed to describe the detailed steps involved in membrane permeation by amphipathic $\alpha$-helical lytic peptides (see Figure 1.11). These models differ conceptually from each other. In the first one, the "barrel-stave" model (Ehrenstein and Lecar, 1977), amphipathic $\alpha$-helices insert into the hydrophobic core of the membrane and form transmembrane pores. In the second one, the "carpet" model (Pouny et al., 1992), the peptides, which do not need necessarily to adopt amphipathic $\alpha$ helical structure, are in contact with the lipid head group during the whole process of membrane permeation and do not insert into the hydrophobic core of the membrane.

Atomic force microscopy (Binnig et al., 1986) offers the possibility to directly image lipid membranes, while the resolution $(0.5-1 \mathrm{~nm})$ is sufficient to directly visualize peptide aggregates. Modern scanning atomic force microscopy (AFM) techniques (Binnig et al., 1986) lend themselves to extend the spatial scale down towards the nanometer range, provided that the membranes can be fixed on solid supports and provided that the lateral structure is reflected in height variations that can be detected by the tip of the AFM under aqueous conditions (Hansma et al., 1994). The need, therefore, arises for a simple, robust, and versatile technique that can be used to directly image by AFM the lateral structure of lipid domains or peptide aggregates by a suitable "amplification" method.

Ivanova et al. (2003) used AFM to search for aggregates of gramicidin A peptide in phosphocholine lipid membranes. Gramicidin A is a small channel forming peptide, which spans the bilayer as a dimer, one monomer in each leaflet, oriented perpendicular to the plane of the membrane. They investigated samples of DMPC (fluid phase) and DPPC (gel phase) lipid bilayers containing gramicidin A between $25^{\circ} \mathrm{C}$ and $30^{\circ} \mathrm{C}$. Two different lipids were used, since DPPC at those temperatures is in the gel phase, whereas DMPC is in the fluid phase. The prediction from calorimetry and Monte Carlo sim- 

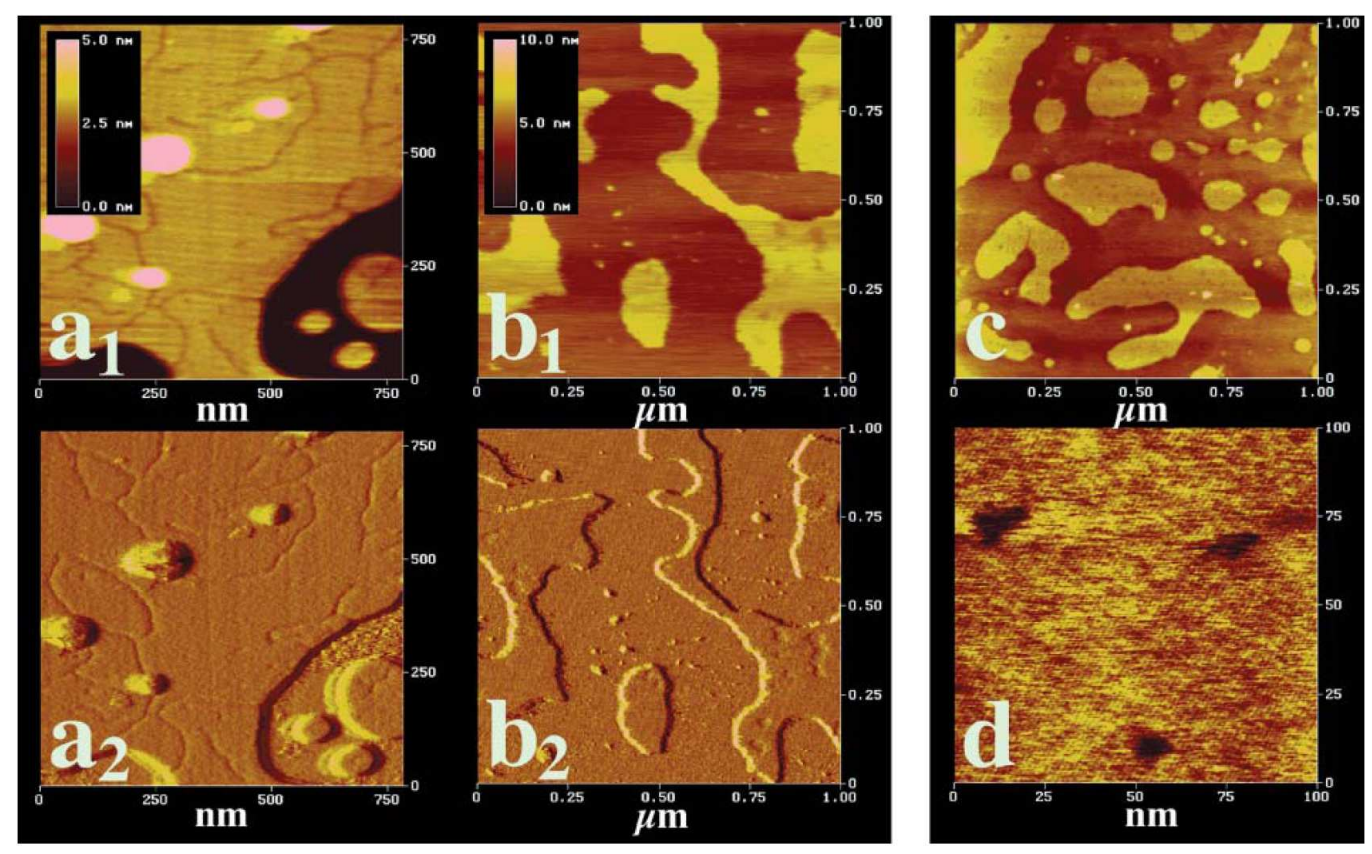

Figure 1.12: Atomic force micrographs of lipid membranes deposited on mica in the presence of gramicidin A. (a1) Height image of 1 mol \% gramicidin A in DPPC membranes in the gel state at $25-30^{\circ} \mathrm{C}$. Linelike depressions can be seen, which scale in length with the peptide content. (a2) Deflection image of panel a1. (b1) Height image of $2 \mathrm{~mol} \%$ gramicidin A in DMPC membranes in the fluid state at $25-30^{\circ} \mathrm{C}$. Small, dotlike spherical depressions can be seen, which scale with the peptide content. (b2) Deflection image of panel a1. (c) Same conditions as in image of panel b1, but different sample. $(d)$ Selected section of the same sample than in panel $c$, with a 10 times smaller scan range. The spherical depressions can be clearly seen. They correspond in area to 50-100 gramicidin dimers. Adapted from Ivanova et al. (2003).

ulations (Ivanova et al., 2003) that gramicidin A aggregates in both lipid phases was supported by AFM (see Figure 1.12). The state of aggregation observed by the AFM differed in the two phases in contrast to the simulations, being line-shaped in the gel phase and being circular in the fluid phase. This is probably caused by the fact that gel phases are two-dimensional crystals (Janiak et al., 1979) and peptide aggregates may rather form at grain boundaries. The fluid phase is rather unordered and no grain boundaries 

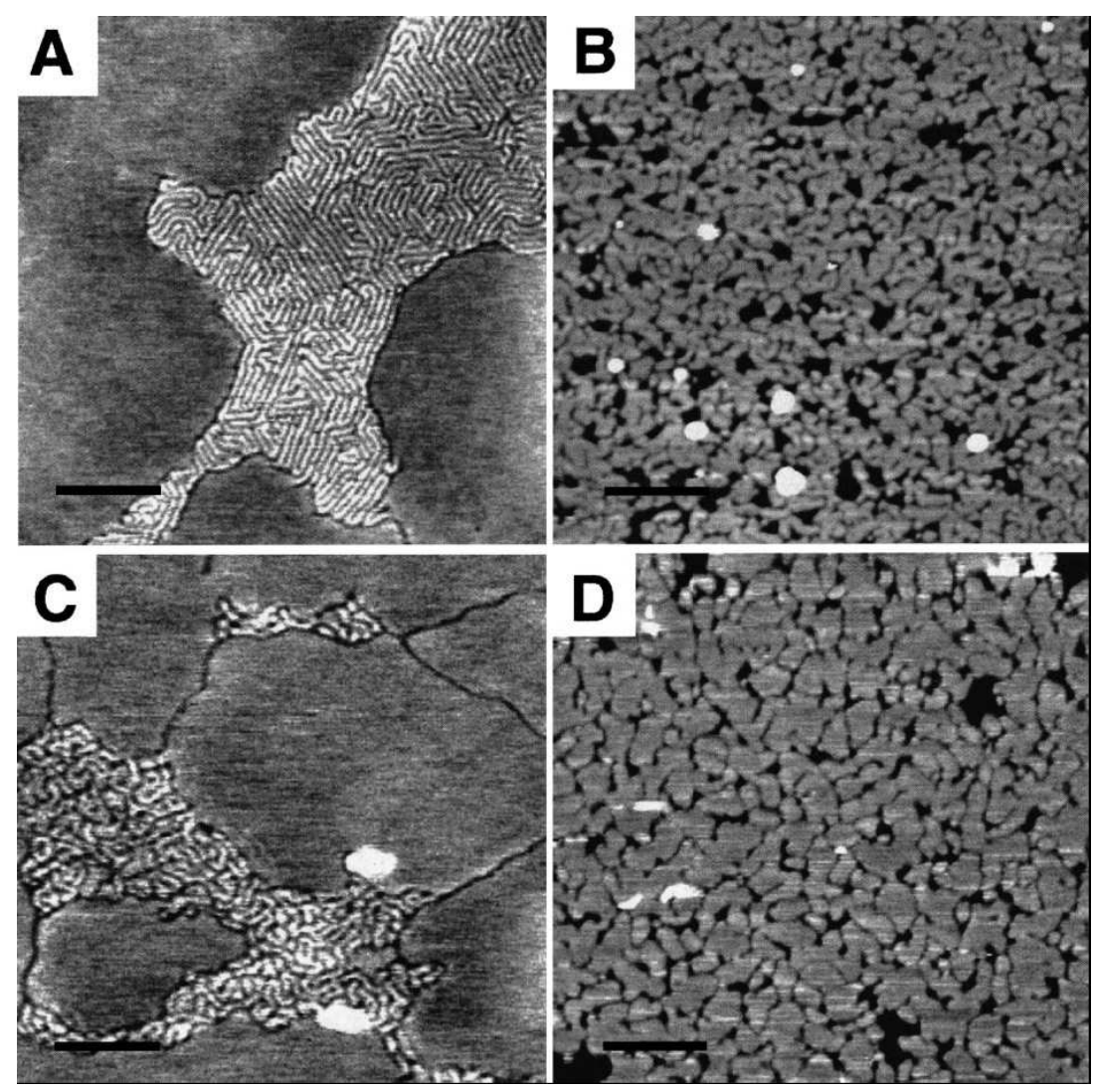

Figure 1.13: DPPC bilayers with 2 mol $\%$ model peptides incorporated. A: WALP23 induces line-type depressions and striated domains exhibiting an ordered pattern. B: KALP23 induces irregularly shaped depressions. C: HALP23 at pH 8 also induces line-type depressions and striated domains. D: At $\mathrm{pH} 5 \mathrm{pH}$ HALP23 induces irregularly shaped depressions. Scale bars for (A) and (C) are $100 \mathrm{~nm}$; (B) and (D) $200 \mathrm{~nm}$; z-scale $3 \mathrm{~nm}$. Adapted from Rinia and de Kruiji (2001).

exist. Thus, aggregation into circular aggregates is likely to be favorable.

Rinia et al. (2000) has imaged aggregation of synthetic, $\alpha$-helical, transmembrane model peptides in phosphocholine lipid bilayers. These so-called WALP peptides consisted of an alternating alanine-leucine (AL) stretch, forming a hydrophobic $\alpha$-helix, flanked by tryptophans (W). A WALP peptide consisting of 23 amino acids in total (WALP23) was incorporated in DPPC bilayers at different peptide concentrations. At 1 mol percent it was found to induce line-type depressions, but in this case, already at this low con- 
centration, small higher domains were present, containing line-type depressions. Upon increasing the peptide concentration, these domains increased in amount and size, from which it was deduced that the peptides were present in the striated domains. The larger domains showed that they consisted of high (light) and low (dark) lines, which formed an extremely ordered pattern exhibiting a three-fold symmetry (see Figure 1.13A). These domains were referred to as striated domains. At $10 \mathrm{~mol}$ percent, the whole bilayer seemed to consist of lines forming this pattern. The repeat distance of these lines, i.e. the width of a low and a high line, was found to be $8 \mathrm{~nm}$. However, peptides flanked by positively charged residues like lysine (KALP) and arginine (RALP), gave rise to a completely different morphology (see Figure 1.13B). They induced line-type depressions and irregularly shaped lower areas, both increasing with increasing peptide concentration, hence, it was concluded that these peptides are present in the depressions and the lower areas. Peptides with histidines (HALP) as flanking residues offer the possibility to see if the change in morphology is due to the presence of positive charges. Histidines are uncharged at high $\mathrm{pH}$ and positively charged at low $\mathrm{pH}$. Indeed, at high $\mathrm{pH}$ HALP peptides induced striated domains, and at low $\mathrm{pH}$ they induced a morphology comparable to the one induced by KALP peptides (see Figure 1.13C,D). This suggested that uncharged peptides are able to induce striated domains in DPPC bilayers, while positively charged peptides are not able to do so.

The AFM experiments shown in Figure 1.14 have revealed that, when the $\mathrm{C}_{14}$-peptide was added to supported gel phase DPPC bilayers from the water phase, it inserts predominantly into pre-existing defect regions in the bilayer and displays a distinct disordering influence on the surrounding lipids. Just after the addition of the peptides the crack zones are markedly affected indicating that the $\mathrm{C}_{14}$-peptide inserts predominantly into crack regions in the supported bilayer and thereby creates local membrane regions having a high peptide concentration. These peptide-enriched regions follow the pre-existing cracks and have approximately a $10 \AA$ smaller height than the surrounding 

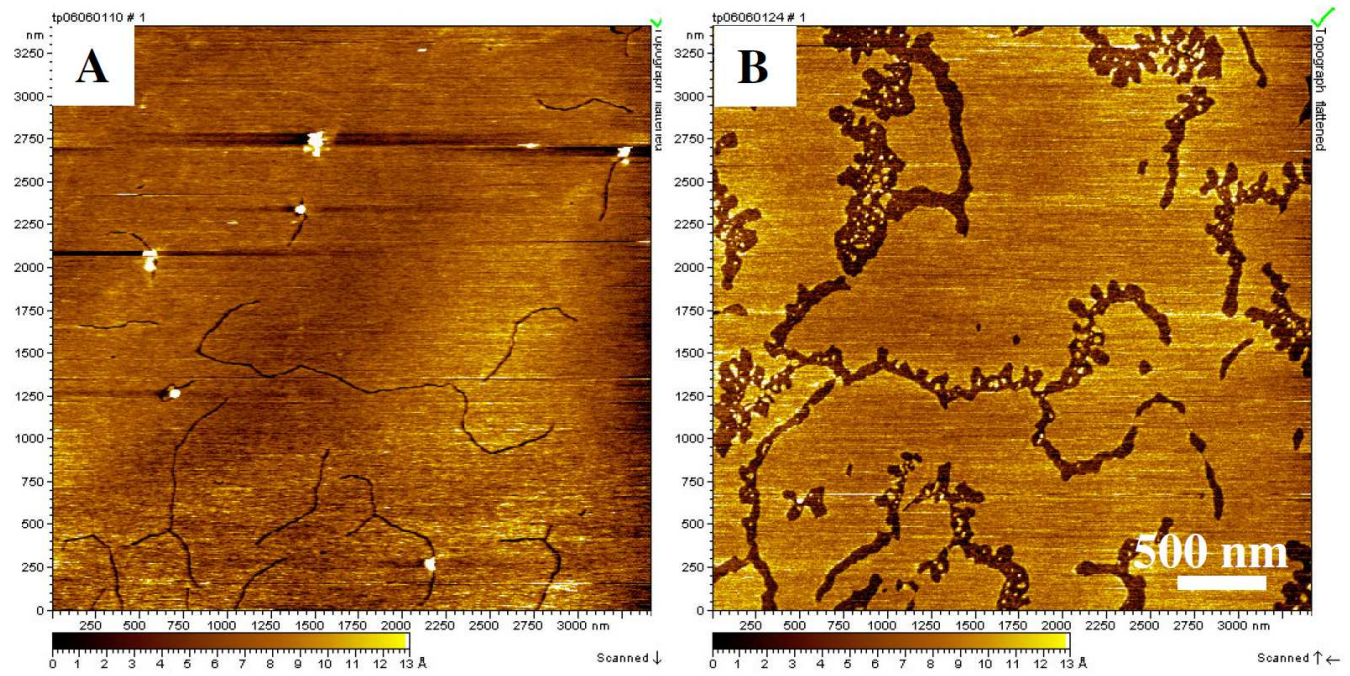

Figure 1.14: (A) AFM micrograph of a DPPC bilayer in the gel phase at $30^{\circ} \mathrm{C}$ on a solid support (mica). Defect lines are visible in the DPPC lipid bilayer. (B) The same DPPC bilayer as in (A) 100 minutes after $\mathrm{C}_{14}$-peptide addition. It is seen that the $\mathrm{C}_{14}$-peptide predominantly inserts into the defect zones and affects the surrounding lipids. Adapted from Kaasgaard (2003).

lipids.

It is known from calorimetry that peptides and proteins influence the chain melting transition of lipid membranes. Usually, transition profiles are broadened and/or shifted to either lower or higher temperatures. The integral band 3 protein of erythrocytes leads to a pronounced broadening of the heat capacity profiles, accompanied by a shift of the $c_{p}$-profiles to higher temperatures (Morrow et al., 1986). The small cyclic peptide gramicidin S, on the other hand, induces a shift of the heat capacity profiles to lower temperatures (Prenner et al., 1999). The shape of the heat capacity profiles contains thermodynamics information on the modes of interaction between peptides and lipids (Heimburg and Biltonen, 1996; Heimburg and Marsh, 1996; Ivanova and Heimburg, 2001), for example about their spatial organization.

Theoretical considerations of peptide-containing lipid membranes, performed previously in our group, allowed to develop a criteria about when to 

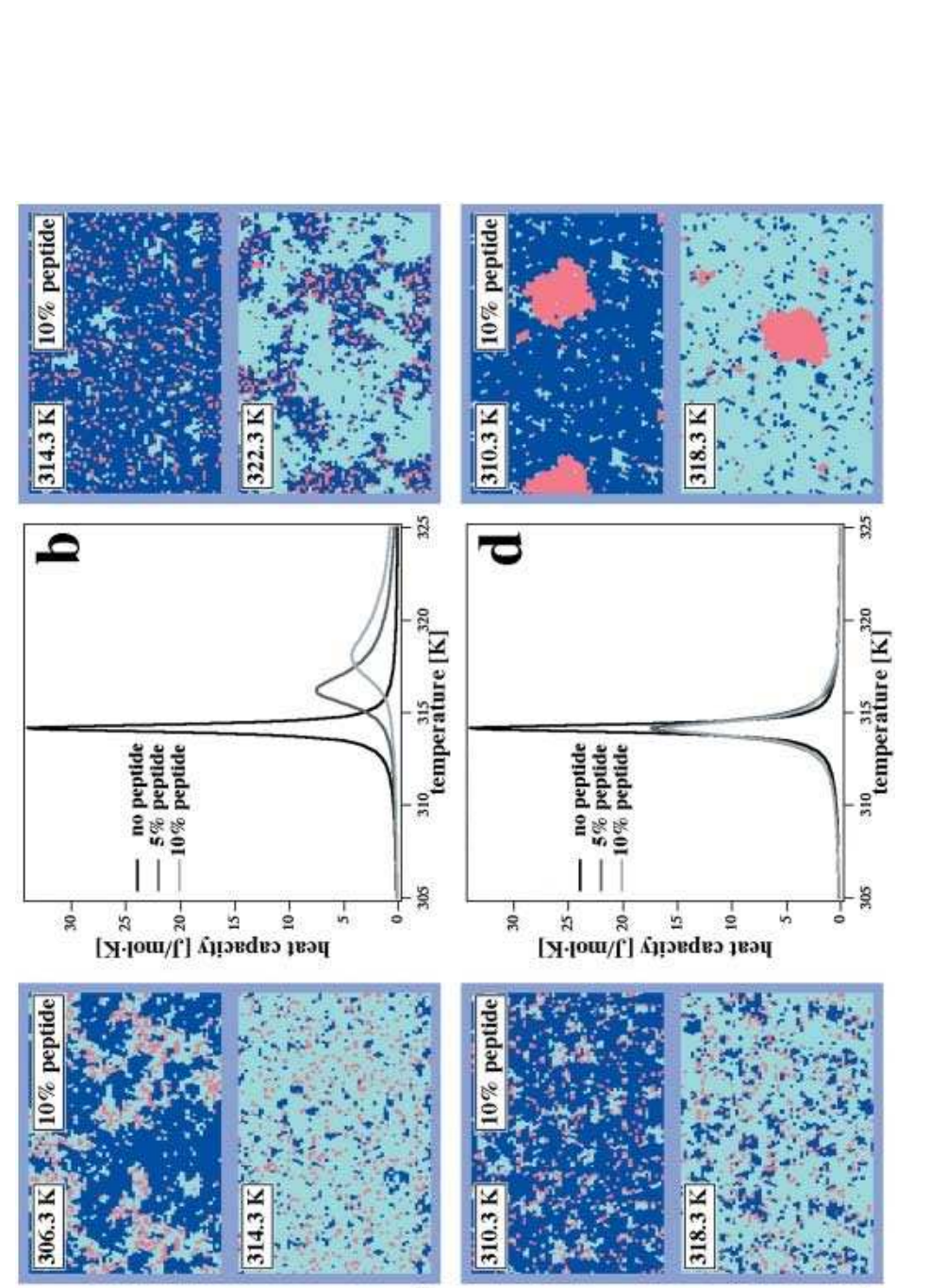

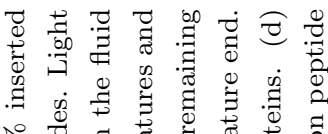

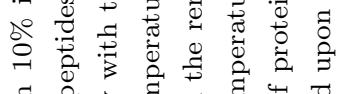

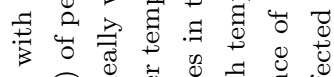

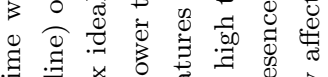

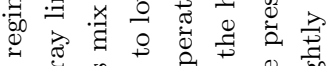
品速

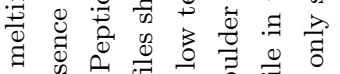

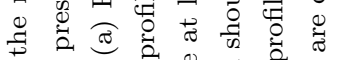

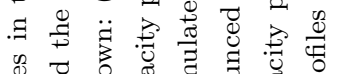

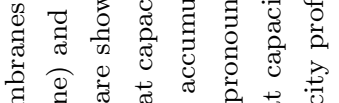

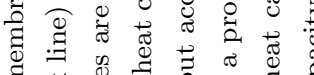
爱 $\because$ :

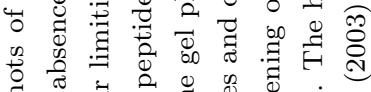

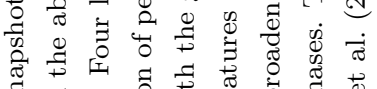

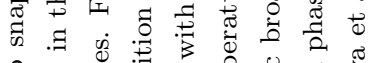

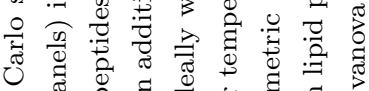
०ूँ

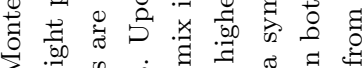

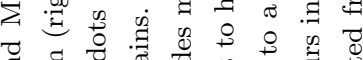

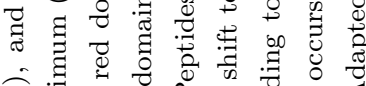

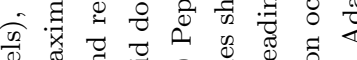

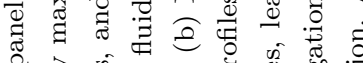

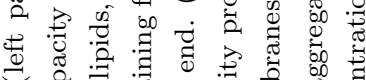

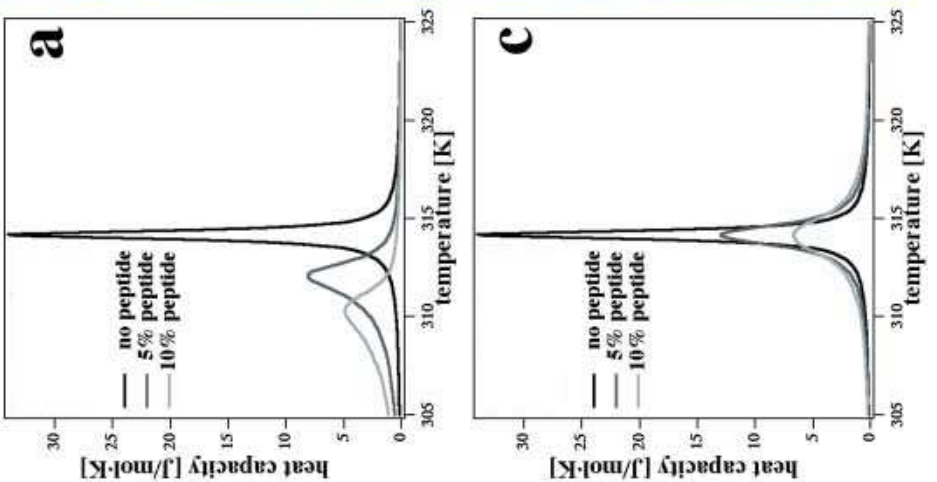

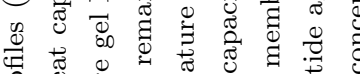

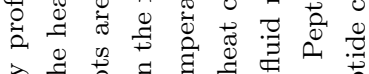

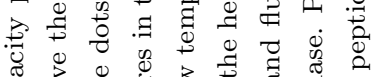

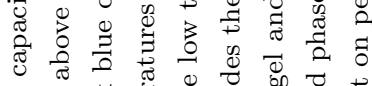

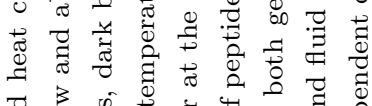

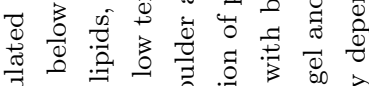

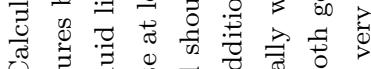

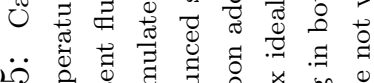

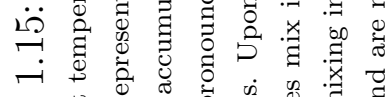

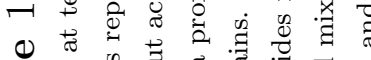

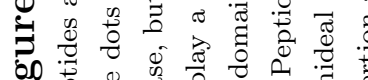

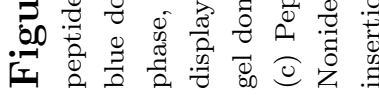


expect peptide clustering (Ivanova and Heimburg, 2001; Ivanova et al., 2003). In that work simulations on lipid peptide systems have been performed using a histogram technique, which is based on extensive Monte Carlo sampling. This technique allows for the calculation of smooth heat capacity profiles, and MC snapshots can serve as a visual representation of the lipid membrane spatial organisation.

In Figure 1.15 four limiting cases of peptide mixing behavior on the heat capacities are shown. If the hydrophobic core of the peptide has a similar length as the thickness of a fluid lipid membrane, a good mixing of the peptide in this phase will result. Upon addition of $5 \%$ or $10 \%$ of an integral peptide (a peptide that inserts into the membrane), melting profiles shift to lower temperatures with an extended wing at the low temperature end (Figure 1.15a, left), accompanied by an accumulation of the peptide in the remaining fluid phase domains (MC snapshots in Figure 1.15a). The shift to lower temperatures is a consequence of the higher peptide-lipid interfacial energy in the gel phase. The asymmetry of the profiles results from the change in mixing behavior. A comparable case is a peptide with a length identical to that of a gel membrane (Figure 1.15b). Under these circumstances, melting profiles shift to higher temperatures and display a wing at the high temperature end.

Even simpler is the case of ideal mixing of peptides in both lipid phases. The effect of the addition of $10 \%$ of peptide on the heat capacity profiles is a symmetric broadening of the heat capacity profiles (see Figure 1.15c). This is due to the fact that peptides located between gel and fluid domains lower the overall line tension and thus the cooperativity of the melting transition.

A further limiting case arises when peptides do not mix well with both phases. The peptides aggregate into clusters at all temperatures (see Figure $1.15 \mathrm{~d}$ ). The effect on the melting profiles is relatively small because the peptides have a reduced interface with the lipids due to aggregation and consequently have a small effect on lipid melting.

It was also shown for the case of small antibiotic peptide gramicidin A 


\section{MC snapshots MC snapshots local}

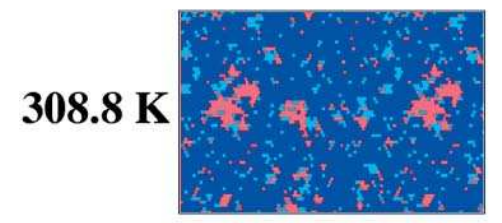
(averaged)

\section{fluctuations}

311.3 K
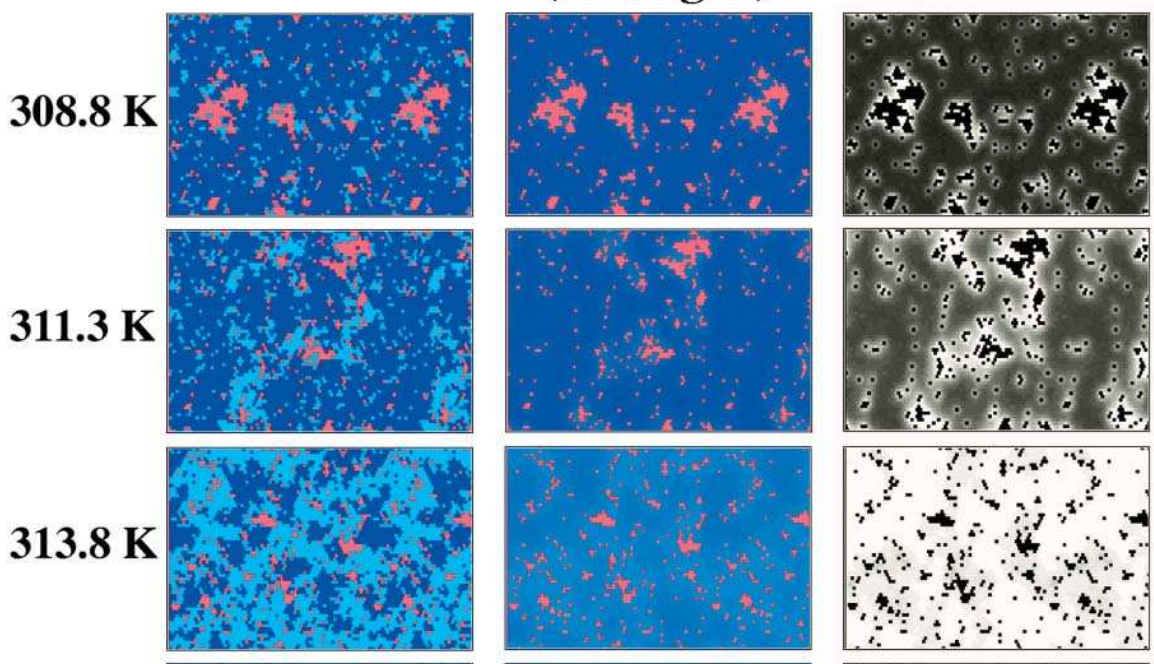

$316.3 \mathrm{~K}$
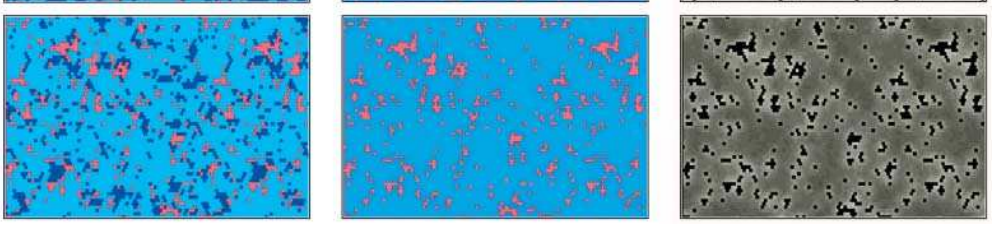

Figure 1.16: MC snapshots, averaged MC snapshots (with peptide diffusion switched off after equilibration), and the corresponding local fluctuations for a DPPC-gramicidin A system with $10 \mathrm{~mol} \%$ peptide at four different temperatures. (Left column) MC snapshots of gramicidin containing system. Gel lipids are shown in dark blue, fluid lipids in light blue, and peptides in red. (Center column) Same as left column, but now averaged over several thousand MC cycles. The lipid states can now vary continuously between gel and fluid state. The average lipid state is different close to large peptide clusters than in the bulk lipid phase. (Right column) Local fluctuations during the simulation. Brighter shades denote larger fluctuations. The color scale varies between 0 (black) and 0.25 (white). The peptide aggregates can be recognized as black areas; size peptides do not display fluctuations in state. It can be seen that the fluctuations close to the peptides are largely altered. This has an immediate impact on the elastic constants close to the peptides. Adapted from Ivanova et al. (2003).

that the fluctuations of lipid state (gel or fluid) in peptide-containing membranes are coupled to the response functions, and it was concluded that compressibility, bending elasticity (or bending stiffness), as well as the relaxation behavior, may be strongly altered close to peptides (Ivanova et al., 2003). Doing Monte Carlo simulations one was able to display the local fluctuations as a function of the lateral coordinates. For the case of gramicidin containing membranes, this is shown in Figure 1.16. Displayed are representative 
MC snapshots (left column), the averaged lipid state (center column), and the local fluctuation profile (right column) at four different temperatures, from below the transition temperature $T_{m}$ to above $T_{m}$. It can be seen that the fluctuations are largely altered close to peptide molecules in a manner strongly dependent on temperature. Gramicidin A slightly shifts the melting temperature to lower values (Ivanova et al., 2003), meaning that the probability to find a fluid lipid close to a peptide is altered (Figure 1.16, center column). Although the fact that the heat capacity events are shifted to lower temperatures already implies a different temperature dependence of the fluctuations, this is a none trivial statement because one now also can resolve the complex spatial distribution of those fluctuations. A further likely effect of high fluctuations close to peptides lies in an increased permeability of the membrane, because the likelihood of spontaneous pore formation should be linked to the lateral compressibility (Makarov, 2005).

In previous studies it was shown that the enthalpy change in lipid gelto-fluid phase transitions is proportional to changes in volume (Anthony et al., 1981; Heimburg, 1998; Ebel et al., 2001). According to the fluctuation/dissipation theorem, the heat capacity is proportional to enthalpy fluctuations, whereas the compressibility is proportional to volume fluctuations. Thus, if enthalpy and volume changes are proportional functions, excess heat capacity and isothermal compressibility are also proportional functions. Furthermore, it was demonstrated that the ratio between enthalpy and volume changes is roughly the same for all studied lipids including complex biological membranes as lung surfactant (Ebel et al., 2001). This leads to the conclusion that the lipid compressibility changes in the melting transition are generally proportional to the excess heat capacity. These findings are of quite some interest because they allow to relate the heat capacity to changes in elastic constants.

It has been shown previously that the bulk ultrasonic velocity of lipid dispersions displays a minimum at the melting transition (Mitaku et al., 1983; Halstenberg et al., 1998; Schrader et al., 2002). The ultrasonic velocity is a 
function of the density and the adiabatic compressibility. The adiabatic volume compressibility, however, is related to the isothermal compressibility via the Maxwell relations. Evaluation of experimental ultrasonic velocity profiles can therefore serve as a tool to obtain information about the compressibility of lipid dispersions. It has been shown for a number of systems like pure lipid suspensions, lipid-cholesterol mixtures, two-component lipid mixtures, that the ultrasonic velocity profiles can be correctly predicted from the heat capacity, if a proportional relationship between heat capacity and isothermal compressibility is assumed (Halstenberg et al., 1998; Schrader et al., 2002).

Let us separate the enthalpy of a hydrated lipid into a bulk term that is independent of the melting process, $H_{0}$, and a differential term, $\Delta H$, describing the change in enthalpy as a function of temperature, $T$, during the melting transition:

$$
\begin{aligned}
H^{\text {lipid }}(T) & =H_{0}^{\text {lipid }}(T)+\Delta H^{\text {lipid }}(T) \\
\frac{\mathrm{d} H^{\text {lipid }}}{\mathrm{d} T} & =\frac{\mathrm{d} H_{0}^{\text {lipid }}}{\mathrm{d} T}+\frac{\mathrm{d}\left(\Delta H^{\text {lipid }}\right)}{\mathrm{d} T}=c_{\mathrm{P}, 0}^{\text {lipid }}+\Delta c_{\mathrm{P}}^{\text {lipid }}
\end{aligned}
$$

Whereas $H_{0}^{\text {lipid }}$ is mainly due to bond vibrations and headgroup hydration, the latter term, $\Delta H^{\text {lipid }}$, describes the changes in chain isomerization and the change in dispersive interactions between chains. $c_{\mathrm{P}, 0}^{\text {lipid }}$ is the heat capacity, and $\Delta c_{\mathrm{P}}^{\text {lipid }}$ is the excess heat capacity. Similarly, one can describe the lipid volume and volume change by the relations

$$
\begin{aligned}
V^{\text {lipid }}(T) & =V_{0}^{\text {lipid }}+\Delta V^{\text {lipid }}(T), \\
\frac{\mathrm{d} V^{\text {lipid }}}{\mathrm{d} T} & =\frac{\mathrm{d} V_{0}^{\text {lipid }}}{\mathrm{d} T}+\frac{\mathrm{d}\left(\Delta V^{\text {lipid }}\right)}{\mathrm{d} T} .
\end{aligned}
$$

For various lipids, lipid mixtures and biological membranes it was experimentally shown (Heimburg, 1998; Ebel et al., 2001) that

$$
\frac{\mathrm{d} V^{\text {lipid }}}{\mathrm{d} T}=\gamma \Delta c_{\mathrm{P}}^{\text {lipid }} \longrightarrow \Delta V^{\text {lipid }}(T)=\gamma \Delta H^{\text {lipid }}(T)
$$

with a proportionality constant $\gamma=7.8 \times 10^{-4} \mathrm{~cm}^{3} / \mathrm{J}^{3}$. The factor $\gamma$ was found to be within experimental error the same for all lipids and mixtures investigated. 
According to the fluctuation-dissipation theorem, the heat capacity is given by the fluctuations of the enthalpy, and the isothermal compressibility is given by the fluctuations of the volume:

$$
c_{\mathrm{P}}=\frac{\bar{H}^{2}-\overline{H^{2}}}{R T^{2}}, \quad \kappa_{\mathrm{T}}=\frac{\bar{V}^{2}-\overline{V^{2}}}{\bar{V} \cdot R T}
$$

where $\mathrm{R}$ is the gas constant, and the overbar denotes thermodynamic averaging. Because of eq 1.3, it follows that

$$
\Delta \kappa_{\mathrm{T}}^{\text {lipid }}(T)=\frac{\gamma^{2} T}{V^{\operatorname{lipid}}(T)} \Delta c_{\mathrm{P}}^{\text {lipid }}
$$

and the total isothermal lipid compressibility is given by

$$
\kappa_{\mathrm{T}}^{\text {lipid }}(T)=f_{\text {gel }}(T) \kappa_{\mathrm{T}, \text { gel }}^{\text {lipid }}(T)+f_{\text {fluid }}(T) \kappa_{\mathrm{T}, \text { fluid }}^{\text {lipid }}(T)+\Delta \kappa_{T}^{\text {lipid }}(T)
$$

where $f_{\text {gel }}$ and $f_{\text {fluid }}$ are the temperature dependent fractions of gel and fluid lipid, respectively, (with $f_{\text {gel }}+f_{\text {fluid }}=1$ ). The fluid fraction can be obtained from integrating the heat capacity profile $f_{\text {fluid }}=\int_{T_{0}}^{T} c_{\mathrm{P}} \mathrm{d} T / \int_{T_{0}}^{T_{1}} c_{\mathrm{P}} \mathrm{d} T$. Here $T_{0}$ is a temperature below, and $T_{1}$ is a temperature above the melting events. $\kappa_{\mathrm{T}, \text { gel }}^{\text {lipid }}(T)$ and $\kappa_{\mathrm{T} \text {,fluid }}^{\text {lipid }}$ are the compressibilities of the pure gel phase and the pure fluid phase. Because fluctuations from different subensembles of a lipid dispersion are additive, the isothermal compressibility of a lipid dispersion is given by the weighted average of the compressibilities of each component of the system

$$
\kappa_{\mathrm{T}}(T)=f^{\mathrm{H}_{2} \mathrm{O}} \kappa_{\mathrm{T}}^{\mathrm{H}_{2} \mathrm{O}}+f^{\text {lipid }} \kappa_{\mathrm{T}}^{\text {lipid }}
$$

where $f^{\mathrm{H}_{2} \mathrm{O}}$ is the volume fraction of water and $f^{\text {lipid }}$ is the volume fraction of lipid. Using the Maxwell relations one can derive the adiabatic compressibility from isothermal compressibility, volume expansion coefficient, heat capacity, and volume:

$$
\kappa_{\mathrm{S}}(T)=\kappa_{\mathrm{T}}(T)-\frac{T}{V c_{\mathrm{P}}(\omega)}\left(\frac{\mathrm{d} V}{\mathrm{~d} T}\right)^{2}
$$

The heat capacity is a function of frequency. The frequency dependence arises from the time dependence of heat exchange within the sample, i.e., absorption 
of heat by chain vibrations, changes in chain conformation, and exchange of heat between lipid and aqueous medium. The latter exchange rates have been measured by volume perturbation calorimetry (Osdol et al., 1989; van Osdol et al., 1991) and pressure jump calorimetry (Grabitz et al., 2002) and were found to be on the millisecond to minute time scale within lipid transitions. This is much slower than the time scale of ultrasonic experiments described below $(2.5 \mathrm{MHz}$ or $\mu$ s time regime).

If one assumes for the time being that there is no heat exchange between the hydrated lipid and the aqueous buffer in the microsecond time regime then the adiabatic compressibility of the lipid dispersion can be considered as an additive parameter (weighted average of the adiabatic compressibility of the water and of the hydrated lipid):

$$
\kappa_{\mathrm{S}}(T)=f^{\mathrm{H}_{2} \mathrm{O}} \kappa_{\mathrm{S}, \mathrm{H}_{2} \mathrm{O}}+f^{\text {lipid }} \kappa_{\mathrm{S}, \text { lipid }} .
$$

Here, the adiabatic compressibility $\kappa_{\mathrm{S}, \mathrm{H}_{2} \mathrm{O}}$ of water has been calculated (Heimburg, 1998) from the volume and the heat capacity of water (Lide and Frederikse, 1996) and the isothermal compressibility (Beggerow, 1980). The adiabatic compressibility of the lipid dispersion is now given by

$$
\kappa_{\mathrm{S}}(T)=f^{\mathrm{H}_{2} \mathrm{O}} \kappa_{\mathrm{S}, \mathrm{H}_{2} \mathrm{O}}(T)+f^{\text {lipid }}\left(\kappa_{\mathrm{T}, \text { lipid }}(T)-\frac{T}{V_{\text {lipid }} c_{\mathrm{P}, \text { lipid }}(\omega)}\left(\frac{\mathrm{d} V^{\text {lipid }}}{\mathrm{d} T}\right)^{2}\right)
$$

with $c_{\mathrm{P}}^{\text {lipid }}=c_{\mathrm{P}}^{0}+\Delta c_{\mathrm{P}}, V_{\text {lipid }}=V_{0}^{\text {lipid }}(T)+\gamma f_{\text {fluid }} \Delta H$. Because heat conduction in water is very high, the adiabatic compressibility of water is nearly identical to its isothermal compressibility in the used frequency regime (around 5 $\mathrm{MHz}$ ). The ultrasonic velocity, $c$, of the lipid dispersion is now given by

$$
c=\sqrt{\frac{1}{\rho \kappa_{\mathrm{S}}}} .
$$

This velocity depends on the lipid fraction $f^{\text {lipid }}$. It can be translated into a function, $u$, which is independent of lipid concentration through

$$
u=\frac{c-c_{\mathrm{H}_{2} \mathrm{O}}}{c_{\mathrm{H}_{2} \mathrm{O}}[L]}
$$


where $[L]$ is the lipid concentration in $\mathrm{mg} / \mathrm{ml}$. Velocity number $u$ reflects the absolute change of the sound velocity of the sample relatively to the reference: negative $u$ values mean smaller sound velocity and positive $u$ values mean larger sound velocity of the sample than the solvent.

The aim of this study is to understand the physical phenomena of lipidpeptide interactions in model membranes of phospholipids with a focus on peptide lateral aggregation processes. This can provide a basis for understanding of peptide and protein biological functions in more complex native biomembranes.

For this purpose several experimental techniques were employed. Differential scanning calorimetry experiments on alamethicin and melittin containing lipid suspensions were performed in order to investigate the influence of these peptides on the lipid membrane phase behavior and to predict the peptide clustering using the above described principles. Sound velocity profiles obtained from ultrasonic measurements of the same systems were used for determining the behavior of the macro compressibility of the membranes in presence of peptides. Using atomic force microscopy the peptides lateral organization in supported lipid membranes and local perturbations of the lipid lattice the induced by them were directly visualized with high spatial resolution. on various peptide concentrations, in the case of alamethicin, and on different lipid states (like gel or fluid), in the case of melittin have been performed. AFM was also used for the imaging of the domain formation in two-component lipid systems. 


\section{Chapter 2}

\section{Materials and methods}

\subsection{Experimental methods}

\subsubsection{Atomic force microscopy}

The atomic force microscope (AFM) is perhaps the most versatile member of a family of microscopes known as scanning probe microscopes (SPM). These instruments generate images by "feeling" rather than "looking" at specimens (Morris et al., 2001; Wiesendanger, 1998). This novel mode of imaging results in a magnification range that correlates with both the light and the electron microscope, but under the "natural" imaging conditions normally corresponds just to the light microscope. The potential to image biological systems in real time, under natural conditions, with molecular, or even submolecular resolution is clearly of interest to biologists and biophysicists.

Since the invention of the AFM (Binnig et al., 1986; Rugar and Hansma, 1990) it has rapidly become a powerful and popular technique in lipid bilayer research, because of its superior resolution and ability to image in aqueous environment. Many different instrumental designs and operating modes of AFM have been developed for mapping of various characteristics of a sample surface: topography, surface conductivity, static charge distribution, localized friction, magnetic fields, viscoelastic properties and others. 
A typical atomic force microscope setup is illustrated in Figure 2.1. The atomic force microscope probes the surface of a sample with a sharp tip, a couple of microns long and often less then $10 \mathrm{~nm}$ in diameter. The tip is located at the free end of a cantilever that is 100 to $200 \mu \mathrm{m}$ long. Forces between the tip and the sample surface causes the cantilever to bend, or deflect. A detector measures the cantilever deflection as the tip is scanned over

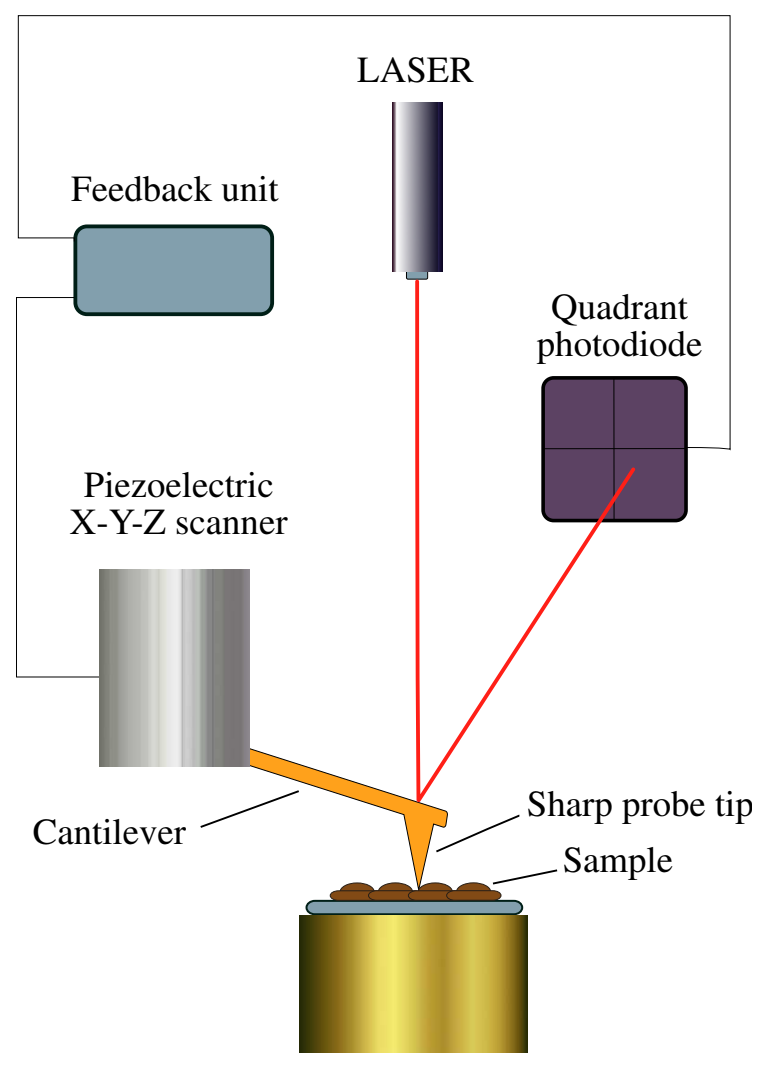

Figure 2.1: The schematic illustration of AFM.

the sample, or the sample is scanned under the tip. The measured cantilever deflections allow a computer to generate a map of surface topography. Most AFMs detect the deflection of cantilever with optical techniques. In the most common scheme, shown in Figure 2.1, a laser beam reflected from the back of the cantilever onto a position-sensitive photodetector. As the cantilever bends, the position of the laser beam on the detector shifts. The photode- 
tector itself can measure displacements of light as small as $10 \AA$. The ratio of the path length between the cantilever and the detector to the length of the cantilever itself produces a mechanical amplification. As a result, the system can detect sub-angstrom vertical movement of the cantilever tip.

The most widely used operating modes of AFM are the so-called contact and tapping mode. They are similar in ability to provide a height information of a sample surface but differ in operating principle and possibility to obtain some additional information.

Contact mode AFM. In contact mode AFM, also known as repulsive mode, an AFM tip makes soft "physical contact" with the sample. The tip is attached to the end of a cantilever with a low spring constant, lower than the effective spring constant holding the atoms of the sample together. As the scanner gently traces the tip across the sample (or the sample under the tip), the contact force causes the cantilever to bend to accommodate changes in topography.

The force most commonly associated with atomic force microscopy is an interatomic force called the van der Waals force. The dependency of the van der Waals force upon the distance between the tip and the sample is shown in Figure 2.2. At the right side of the curve the atoms are separated by a large distance. As the atoms are gradually brought together, they first weakly attract each other. This attraction increases until the atoms are so close together that their electron clouds begin to repel each other electrostatically. This electrostatic repulsion progressively weakens the attractive force as the interatomic separation continues to decrease. The force goes to zero when the distance between the atoms reaches a couple of angstroms, about the length of a chemical bond. When the total van der Waals force becomes positive (repulsive), the atoms are in contact.

The slope of the van der Waals curve is very steep in the repulsive or contact regime. As a result, the repulsive van der Waals force balances almost any force that attempts to push the atoms closer together. In AFM 


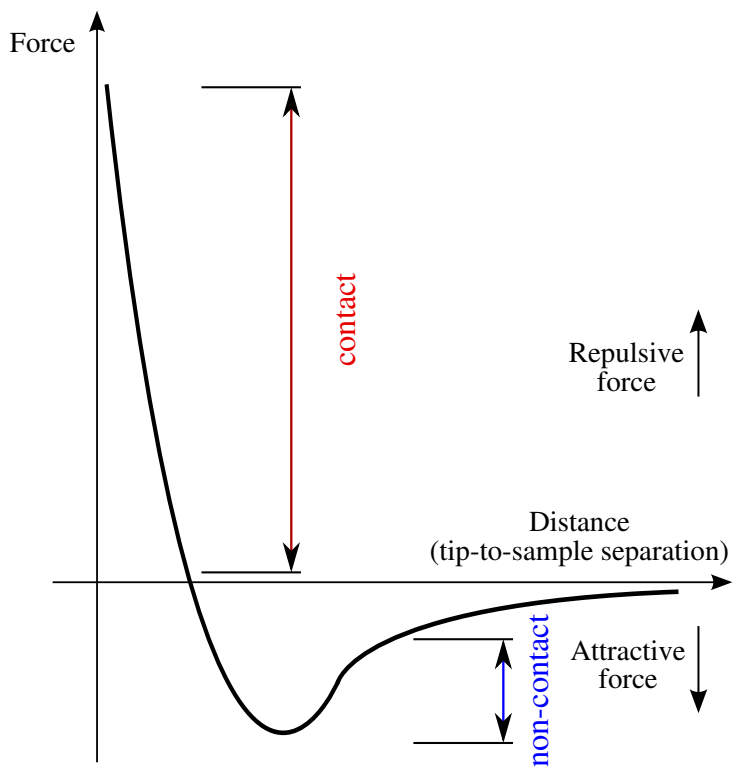

Figure 2.2: Interatomic force vs. distance curve.

this means that, when the cantilever pushes the tip against the sample, the cantilever bends rather than forcing the tip atoms closer to the sample atoms. Even if you design a very stiff cantilever to exert large forces on the sample, the interatomic separation between the tip and sample atoms is unlikely to decrease much. Instead, the sample surface is likely to deform.

As long as the tip is in contact with the sample, the variable force in contact AFM is the force exerted by the cantilever. The total force that the tip exerts on the sample must be balanced by the repulsive van der Waals force for the contact AFM. The magnitude of the force exerted on the sample varies from $10^{-8} \mathrm{~N}$ to the more typical operating range of $10^{-7}$ to $10^{-6} \mathrm{~N}$.

Before initiating an AFM scan in contact mode, the tip is brought into contact with the sample which causes the cantilever to deflect until a preset deflection is reached. During scanning this pre-set cantilever deflection is kept constant by a feedback loop that vertically retracts and extends the piezoelectric scanner (See Figure 2.1) in response to height changes that the tip encounters. In this way the force that the tip applies to the sam- 
ple remains constant as the tip traces the sample topography. Plotting the vertical displacement of the piezoelectric element versus the $\mathrm{x}-\mathrm{y}$ coordinates generates the AFM topography, or "height" image. In spite of the fact that topographic AFM-images are 3-dimensional, they are commonly displayed as 2-dimensional images with a color scale assigned to height differences. A typical color scale is shown in Figure 2.3 where dark colors correspond to low features and bright colors correspond to high features. Keeping the can-

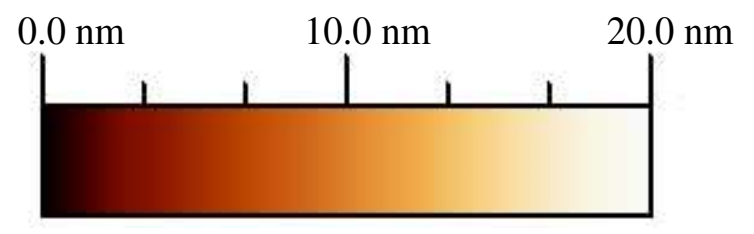

Figure 2.3: A typical color scale used for topographic AFM-images.

tilever deflection constant during scanning corresponds to an ideal situation that cannot be achieved in practice. In practice, there is a small time-lag before the feedback loop adjusts to the height changes that the tip encounters. The difference between the actual cantilever deflection and the desired deflection is called the error signal, or deflection signal, and is often plotted as an image along with the topographic image. Deflection images highlight edges where drastic height changes occur but do not otherwise contain useful height information.

Tapping mode AFM. Tapping mode imaging (Zhong et al., 1993; Hansma et al., 1994) overcomes the limitations of the conventional scanning modes by alternately placing the tip in contact with the surface to provide high resolution and then lifting the tip off the surface to avoid dragging the tip across the surface. Tapping mode is implemented by oscillating the cantilever assembly at or near the cantilever's resonance frequency using a piezoelectric crystal. The piezo motion causes the cantilever to oscillate with a high amplitude (the "free" amplitude, typically bigger than $20 \mathrm{~nm}$ ) when the tip is not in contact with the surface. The oscillating tip then moved toward 


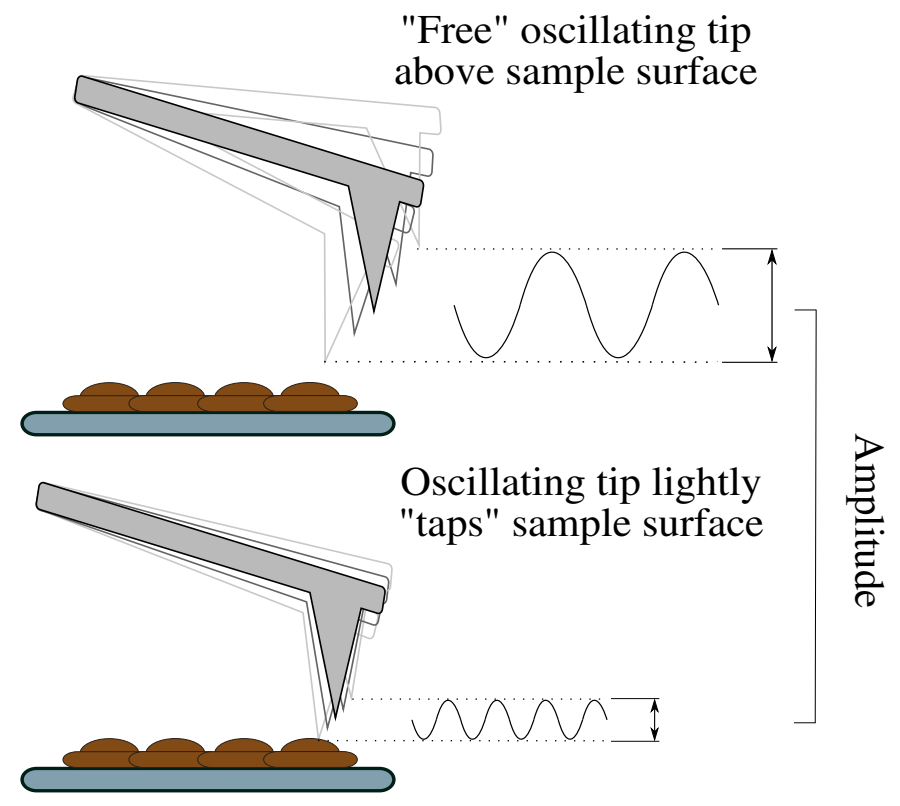

Figure 2.4: Vibrating tip in the tapping mode lightly taps the surface of the sample. The amplitude of tip oscillations is related to the strength of the force applied to the sample surface.

the surface until it begins to scflightly touch, or "tap" the surface. During scanning, the vertically oscillating tip alternately contacts the surface and lifts off, generally at a frequency of $10^{4}$ to $5 \cdot 10^{5} \mathrm{~Hz}$. As the oscillating cantilever begins to intermittently contact the surface, the cantilever oscillations necessarily reduced (See Figure 2.4) due to energy loss caused by the tip contacting the surface. The reduction in oscillation amplitude is used to identify and measure surface features. During tapping mode operation, the cantilever oscillation amplitude is maintained constant by a feedback loop. When the tip passes over a bump in the surface, the cantilever has less space to oscillate and the amplitude of oscillation decreases. Conversely, when the tip passes over a depression, the cantilever has more room to oscillate and the amplitude increases (approaching the maximum "free" amplitude). The oscillation amplitude of the tip is measured by the photodetector and the photodetector signal is input to the AFM controller electronics. The feedback loop then adjusts the tip-sample separation to maintain a constant 


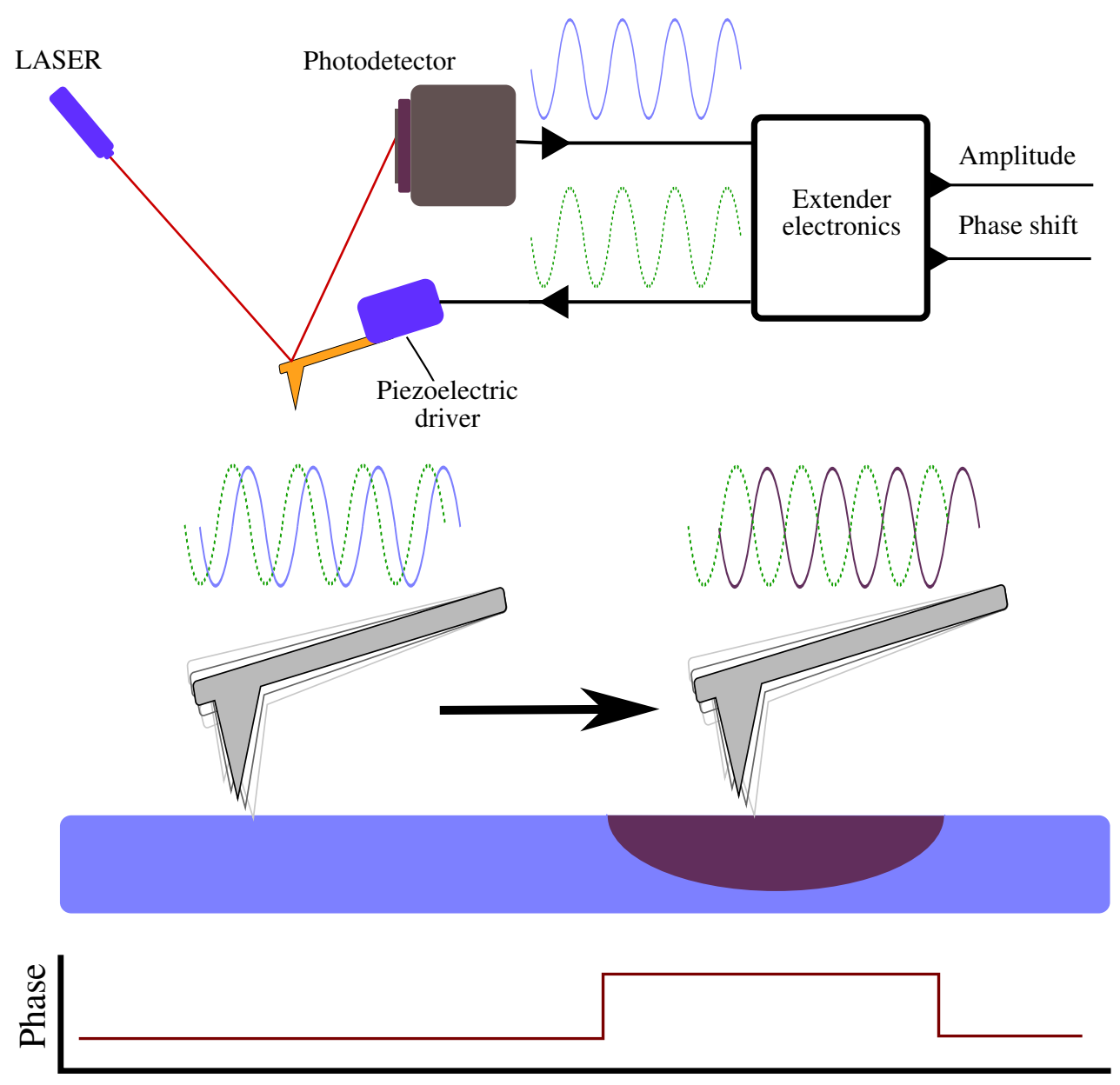

Figure 2.5: Illustration of phase imaging. Variations of phase delay between the oscillation of the driving piezo and the oscillation of the tip are related to variations of sample surface properties.

amplitude. Plotting the vertical displacement of the piezo versus the $\mathrm{x}-\mathrm{y}$ coordinates generates the AFM image.

Keeping the cantilever oscillation amplitude constant during scanning corresponds to an ideal situation which is not achieved during experiment. Therefore similar to contact mode, in tapping mode one can obtain an analog of "deflection image" namely an "amplitude image" which highlights edges where drastic height changes occur, but do not otherwise contain useful height information. 
Imaging in tapping mode one can also obtain a so-called "phase image" of the sample surface. Phase imaging is a technique that can be used to map variations in surface properties such as elasticity, adhesion, composition and friction. Phase detection refers to the monitoring of the phase lag between the signal that drives the cantilever to oscillate and the cantilever oscillation output signal (See Figure 2.5). Changes in the phase lag reflect changes in the mechanical properties of the sample surface. The system's feedback loop operates in the usual manner, using changes in the cantilever's vibration amplitude to measure sample topography. The phase lag is monitored while the topographic image is being taken so that images of topography and material properties can be collected simultaneously. Although there is currently no simple correlation between phase contrast and a single material property, one can often clearly distinguish which of the mentioned surface properties makes the greatest contribution to the phase shift between the driving force and cantilever response (Argaman et al., 1997; Hansma et al., 1997; Sauer et al., 1998; Pang et al., 2000).

In the present work we used several AFM microscopes produced by Digital Instruments (Santa Barbara, CA, USA) such as MultiMode, BioScope and EnviroScope. The BioScope microscope was found not suitable for the topography imaging of supported lipid bilayers in fluids due to the low signalto-noise ratio. Two others, MultiMode and EnviroScope, produced satisfactory images with scan sizes up to $800 \mathrm{~nm}$. Using the MultiMode microscope yielded height and phase images of better quality in the tapping mode in comparison with the EnviroScope microscope.

\subsubsection{Differential scanning calorimetry}

Differential scanning calorimetry (DSC) is a technique (Plotnikov et al., 1997) for measuring the energy necessary to establish a nearly zero temperature difference between a substance and an inert reference material, as the two specimens are subjected to identical temperature regimes in an environment heated or cooled at a controlled rate. 


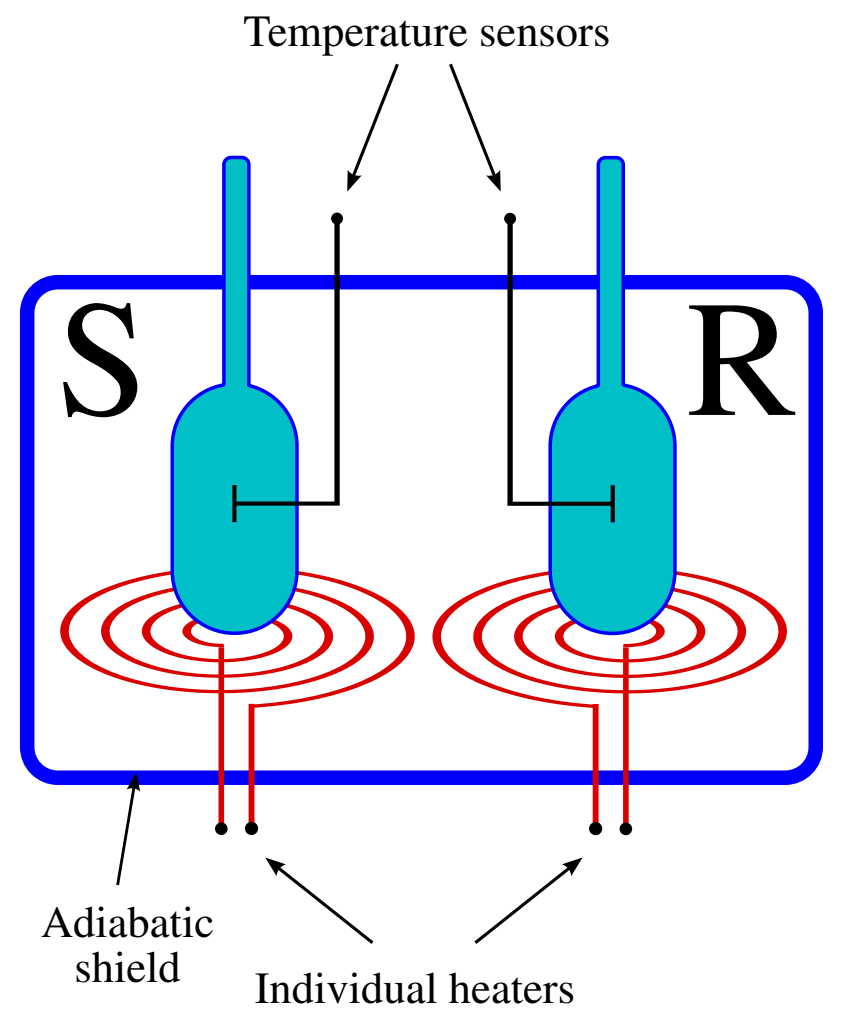

Figure 2.6: Principle scheme of a differential scanning calorimeter.

This technique is of primary importance in obtaining information about the thermodynamics of model membranes and biomembranes. It is used to monitor and characterize changes in the physical state in polymorphic lipids and also to characterize the perturbations on pure lipids by the interactions with other materials, such as other lipids, proteins, peptides, ions, or small hydrophobic molecules. In a differential scanning calorimeter, the sample and the inert reference are heated independently to maintain an identical temperature in each. The heat for the endothermic gel-to-fluid bilayer transition, for example, would be required in excess over the heat required to maintain the same temperature in the reference. Differential heat flow is then plotted as a function of temperature. Highly sensitive instruments allow one to use samples of dilute aqueous suspensions of lipids $(1 \mathrm{mg} / \mathrm{ml}, 1$ $\mathrm{ml}$ sample volume). The parameters reported from this technique for lipid 
systems are:

1. Transition midpoint temperature, $T_{m}$ : where the transition is 50 per cent complete.

2. Transition enthalpy, $\Delta \mathrm{H}$ : the actual heat required for the entire transition normalized per mole or per unit weight.

3. Heat capacity, $C_{p}$ : the amount of heat (per gram or per mole) required to raise the temperature of the sample by a given temperature increment.

A differential scanning calorimeter consists of two cells (See Figure 2.6), one containing the sample and the other the inert reference material, which can be heated at a programmed rate by heaters controlled to maintain the temperature difference between the two cells to equal zero. If the sample is a solution or a suspension, the reference material is normally the corresponding solvent. When a thermally initiated process takes place in the sample cell, the control system responds by increasing or reducing the heat supply to the sample cell so that its temperature is equal to that of the reference cell. The data output of the calorimeter is the corresponding excess power, presented as a function of the temperature. It is worth noting that a heat evolution in the sample can only be observed if the thermally induced reaction is kinetically limited as compared to the heating rate of the scan, because a temperature increase shifts the thermodynamic equilibrium in an endothermic direction.

For our measurements a very sensitive differential scanning calorimeter VP-DSC from MicroCal (Northhampton, MA, USA) was used. The device records the excess power $\Delta P$, the time $t$ and the temperature $\hat{T}$. Then the excess heat, $\Delta Q$, required for the temperature change and the heat capacity difference, $\Delta C_{p}$, (at constant pressure $p$ ) between the sample and the reference are computed.

$$
\Delta Q=\int_{t}^{t+\Delta t} \Delta P\left(t^{\prime}\right) d t^{\prime} \cong \Delta P \cdot \Delta t
$$




$$
\Delta C_{p}=\left(\frac{\partial Q}{\partial T}\right)_{p} \cong \frac{\Delta Q}{\Delta \hat{T}}=\frac{\Delta P}{\left(\frac{\Delta \hat{T}}{\Delta t}\right)}
$$

where $(\Delta \hat{T} / \Delta t)$ is the scan rate. The two cells are made of tantalum. The calorimeter used has a wide spectrum of scan rates $(0 \ldots 60 \mathrm{deg} / \mathrm{h})$, a large signal-to-noise ratio and a very stable base line.

\subsubsection{Ultrasonic spectrometry}

During the past decades ultrasonic spectrometry (often named spectroscopy) in liquids has become an important tool for basic and applied research in physics, physical chemistry, material science, biology and last but not least medicine. Application of ultrasonic spectrometry provides a researcher with valuable thermodynamical parameters of lipid bilayer membranes. In the present work we have measured ultrasonic velocity profiles which havebeen evaluated to obtain information about the compressibility of peptidecontaining lipid vesicle dispersions (Schrader et al., 2002).

Experiments were performed using a so-called resonator method. The principle of resonators is based on "folding" or convoluting the acoustic path via multiple reflections at low liquid attenuation, in order to increase the effective sonic pathlength and to obtain a resolvable amplitude decay. The cross section of an ultrasonic resonator cell built in our lab is shown in Figure 2.7. Two piezoelectric transducers confine a cavity, containing the liquid, and establish a compound resonator. This cell is symmetrical; therefore input and output can be exchanged. Nearly planar waves, excited and detected by disc-shaped, planar X-cut quartz transducers, propagate back and forth in the liquid. Resonances are indicated by voltage peaks in the RF output from the receiving transducer.

The sound velocity of the sample at around $2.5 \mathrm{MHz}$ was derived from the resonance frequencies of the cell, which in turn were determined from the continuously recorded resonator transfer functions. A simplified analysis can be applied demonstrating how to obtain the sound velocity from the data 


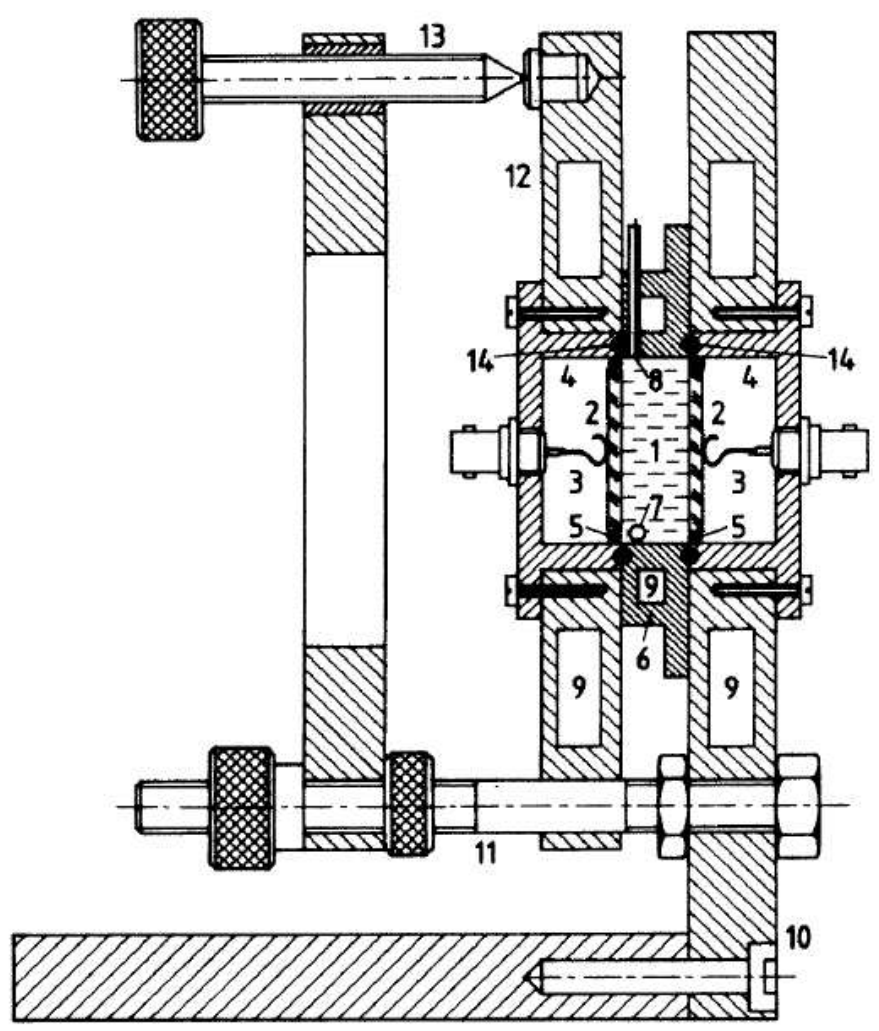

Figure 2.7: The cross section of a fixed-path cell, built in our lab: 1, sample cavity; 2, piezo-electric transducer disc; 3, flexible electrical contact wire; 4, exchangeable transducer mount; 5 , sealing ring (such as an adhesive layer of silicone rubber) with embedded electrical contacts for the earthed front electrode of the transducer; 6 , thermostatted cell jacket (transparent material permits inspection of gas bubbles); 7 and 8 , inlet and outlet, respectively, for the liquid sample cavity; 9, circulation channel for the thermostat fluid; 10, main frame and 11, screw bolts, which permit a variation of pathlength by an exchange of jacket $6 ; 12$, adjustable frame plate; 13, adjustment screws for transducer parallelism; 14, sealing O-ring. The thermostatted outer shield is not shown. Illustration was taken from Eggers and Kaatze (1996).

provideded by an ultrasonic resonator. The essential condition of establishing coincident waves in the resonator is the equality of the acoustic path $L$ to the whole number of the half wavelengths of a propagating wave: 


$$
L=n \cdot\left(\lambda_{n} / 2\right)
$$

where $n=1,2,3, \ldots, N$. Taking into account the relation between the wave length $\lambda$ and the frequency $(f=c / \lambda)$ one obtains the equation for the eigenfrequencies $f_{S n}$ of resanator:

$$
f_{S n}=\frac{c_{S} \cdot n}{2 \cdot L}
$$

where $c_{S}$ is the sound velocity of the sample liquid filled between trancducers separated by the distance of $L$ and $f_{S n}$ is the frequency of the $n$-th resonance. In order to determine the trancducers separation $L$ we measured the resonance frequencies of a substance with well known sound velocity, in our case water. Thus the sound velocity of the sample can be determined as follows:

$$
L=\frac{c_{W} \cdot n}{2 f_{W}} \quad \Rightarrow \quad c_{S}=c_{W} \frac{f_{S n}}{f_{W n}}
$$

This simple formula is well acceptable for determining the sound velocity of diluted lipid suspensions. A more complicated analysis which also considers the properties of piezo trancducers and the particularities of wave reflections is described in (Eggers and Kaatze, 1996).

\subsection{Materials and sample preparation}

\subsubsection{Substances: lipids and peptides}

\section{Melittin}

The venom of the European honey bee Apis mellifera contains as its major protein component a 26 amino acid peptide, called melittin, which has a powerful hemolytic activity (Dempsey, 1990; Bechinger, 1997; Dathe and Wieprecht, 1999).

The amino acid sequence is:

$$
\begin{gathered}
\text { H-Gly-Ile-Gly-Ala-Val-Leu-Lys-Val-Leu-Thr-Thr-Gly-Leu-Pro- } \\
\text { Ala-Leu-Thr-Ser-Trp-Ile-Lys-Arg-Lys-Arg-Gla-Gla-N } \mathrm{H}_{2}
\end{gathered}
$$



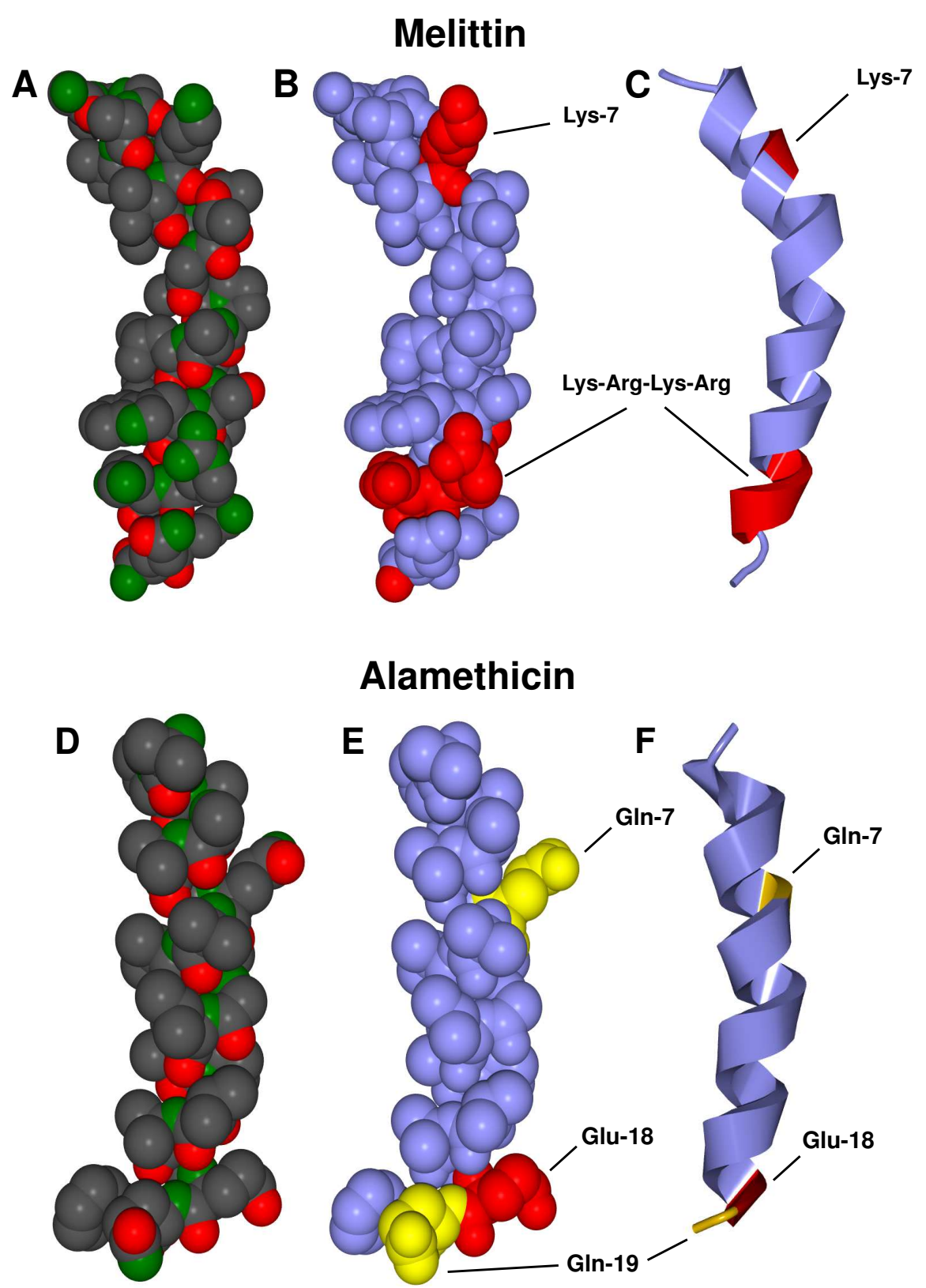

\section{Alamethicin}

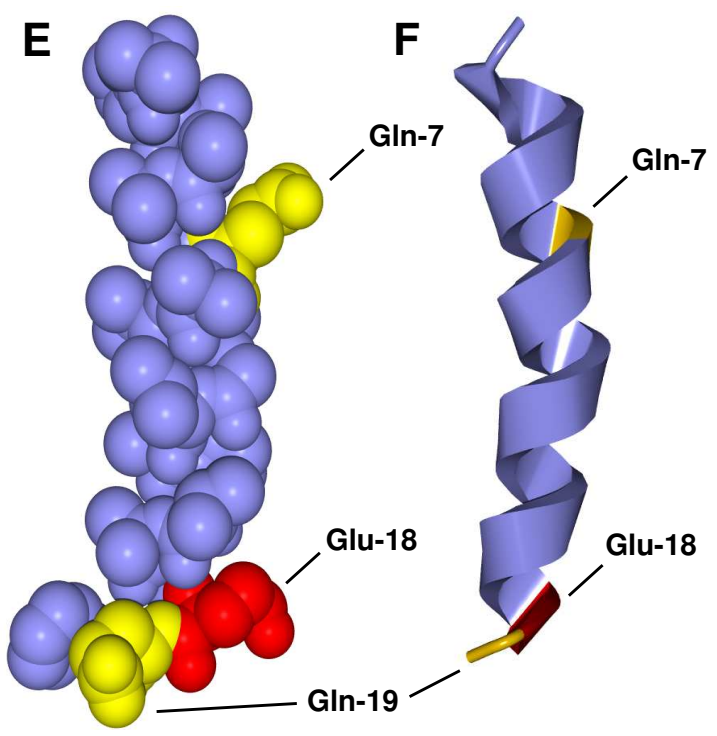

Figure 2.8: Crystal structure of melittin ${ }^{1}$ and alamethicin molecules. On $\mathbf{A}$ and D: black color corresponds to carbon atoms, red to oxygen and green to nitrogen. Hydrogens are not shown. Important residues of melittin (B) and of alamethicin (E) implicated to be important for pore-formation. Charged residues highlighted in red, polar in yellow, and all other hydrophobic residues in light blue. Helical structures of both peptides are schematically depicted on $\mathbf{C}$ and $\mathbf{F}$, respectively. 
In common with other membrane-binding peptides and membrane proteins, melittin is predominantly hydrophobic. The peptide has a net charge of +6 , four of these charges are in the highly basic C-terminal tetrapeptide sequence Lys-Arg-Lys-Arg, with only the N-terminal amino group and Lys7 in the N-terminal sequence. Skewed distribution of polar and non-polar amino acids is apparent if the peptide is placed in an $\alpha$-helical configuration (see Figure 2.8). The polar amino acids in the first 21 residues lie on one face of the helix with the non-polar amino acids segregated almost completely on an opposite side of the helix. This so-called amphiphilic nature is a characteristic of many membrane-binding peptides and of transmembrane helices of many membrane proteins.

Melittin was purchased as a powder from Alexis Biochemicals (San Diego, CA, USA).

\section{Alamethicin}

Alamethicin is a 20-residue peptide from the fungus Trichoderma viride which contains a high proportion of the unusual amino acid $\alpha$-aminoisobutyric acid (Aib), in addition to two proline residues, and is capable of forming voltage-gated channels of very high conductance in lipid membranes (Cafiso, 1994; Marsh, 1996; Duclohier and Wróblewski, 2001).

The amino acid sequence is:

$$
\begin{gathered}
\text { Ac-Aib-Pro-Aib-Ala-Aib-Ala-Gln-Aib-Val-Aib- } \\
\text { Gly-Leu-Aib-Pro-Val-Aib-Aib-Gln-Glu-Phl }
\end{gathered}
$$

Such peptaibol peptides which contain several Aib residues have a strong propensity to form helices. The X-ray crystal structure determined for alamethicin shows it to be predominantly $\alpha$-helical with a bend, punctuated by a partial 310 -helical turn (see Figure 2.8), that is generated by the proline

\footnotetext{
${ }^{1}$ Molecular structures of melittin (Gribskov et al., 1990) and alamethicin (Fox and Richards, 1982) were reproduced using Swiss-PdbViewer software from pdb-files available online from http://www.rcsb.org/pdb/ .
} 
residue at position 14. The molecule of alamethicin is highly hydrophobic but there are two polar residues Gln-7 and Gln-19 and one charged residue Glu-18. They have their side chains extended from the convex face of the helix, and the presence of Pro-14 causes the backbone carbonyls of Aib-10 and Gly-11 also to be exposed on this face. The latter increases the amphiphilic nature of the helix and contributes to its channel-forming properties.

Alamethicin was provided by Sigma (St. Luis, MO, USA).

\section{Lipids}

The used lipids (see Table 2.1) were purchased as powder from Avanti Polar Lipids (Birmingham, AL) and were used without further purification. All

\begin{tabular}{|c|c|}
\hline $\begin{array}{c}\text { DLPC } \\
621.89 \mathrm{~g} / \mathrm{mol}\end{array}$ & $\begin{array}{l}\text { 1,2-dilauroyl-sn-glycero-3-phosphocholine } \\
\text { dilauroylphosphatidylcholine }\end{array}$ \\
\hline $\begin{array}{c}\text { DMPC } \\
677.94 \mathrm{~g} / \mathrm{mol}\end{array}$ & $\begin{array}{c}\text { 1,2-dimyristoyl-sn-glycero-3-phosphocholine } \\
\text { dimyristoylphosphatidylcholine }\end{array}$ \\
\hline $\begin{array}{c}\text { DPPC } \\
734.05 \mathrm{~g} / \mathrm{mol}\end{array}$ & $\begin{array}{c}\text { 1,2-dipalmitoyl-sn-glycero-3-phosphocholine } \\
\text { dipalmitoylphosphatidylcholine }\end{array}$ \\
\hline
\end{tabular}

Table 2.1: Abbreviation, systematic name, molar mass and a common synonym of the used lipids.

lipids are phosphocholines that differ one from another just by the number of carbon atoms in fatty acid chains. Having very similar chemical and physical properties they have very different gel-to-fluid transition temperatures. Since the AFM microscopes were not able to do temperature dependent imaging, different lipids were taken depending from their melting point temperature in 
order to investigate peptide-containing membranes in the gel and fluid states at room temperature conditions.

\subsubsection{Preparation of multilamellar vesicle suspensions}

For the preparation of lipid-lipid or lipid-peptide multilamellar dispersions both lipids and peptides were separately dissolved in a 1:1 mixture of and methanol. The dissolving of lipids and peptides in the organic solvents preceding the preparation of aqueous solutions was required for two reasons: 1) for more exact weighing of the substances in micrograms amounts and 2) for better mixing of peptides and lipids for the peptide-containing samples or lipid-I and lipid-II molecules for the two component lipid samples. Further, appropriate amounts of the solutions of the target substances were mixed together and dried under a weak flow of nitrogen gas. In order to remove the rest of organic solvents dried films were kept overnight under low pressure. After that, dry samples were dispersed to a final concentration in EDTA:HEPES (1:10) buffer with pH 7.4. Aqueous multilamellar lipid dispersions were prepared by heating the samples to the tempreture of $50-65^{\circ} \mathrm{C}$ followed by vortexing.

\subsubsection{Sample preparation for AFM experiments}

For the preparation of samples for AFM experiments we used the direct fusion of small unilamellar vesicles on mica (Shao and Yang, 1995). Freshly cleaved mica is an excellent substrate with few defects present and free of contaminations. Small unilamellar vesicles (SUVs) were prepared by intense sonication (see Figure 2.9) of the multilamellar suspensions using a SONIFIER Cell Disruptor B-15 provided by Branson (Geneve, Switzerland). Power control (the amplitude of the titanium tip vibrations) and cycling duty (relative time of the tip vibrations) parameters of the sonifier were chosen between 1-3 units and 20-50\%, respectively, depending from the volume and lipid concentration of the samples. Multilamellar suspensions were sonified until 


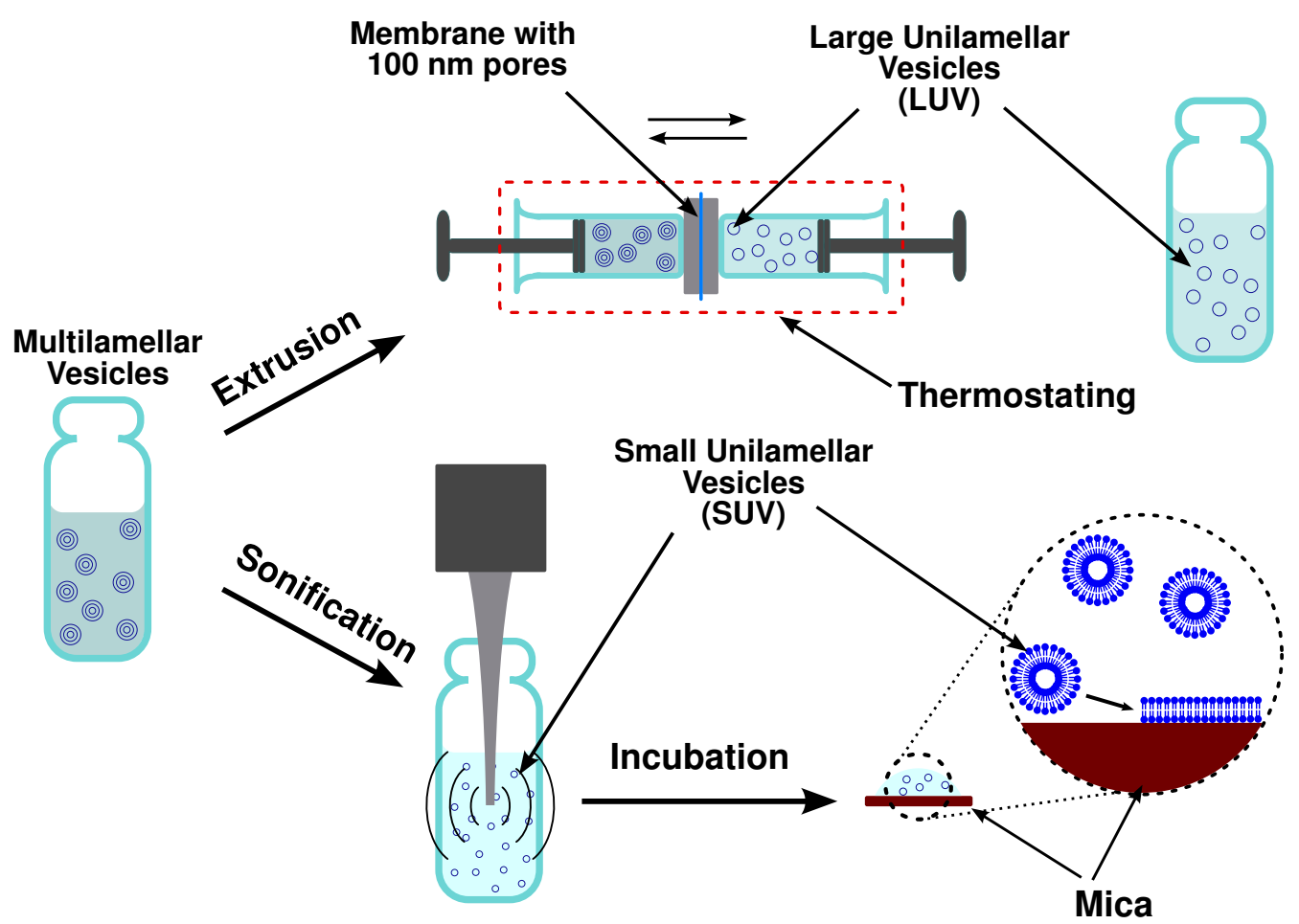

Figure 2.9: Preparation of large unilamellar vesicles for calorimetry and ultrasonic experiments using the extrusion procedure and supported lipid bilayers for AFM-experiments using the direct fusion of small unilamellar vesicles on mica.

they changed their opacity from "milky" white to completely transparent. For instance, in the case of the DPPC $5 \mathrm{mM}$ sample of $2 \mathrm{ml}$ volume, the power control parameter was adjusted to 1 unit and the cycling duty to $30 \%$, and 20 min of the sonification was found to be sufficient. The SUVs were immediately warmed up to the temperature above the phase transition of lipids otherwise vesicles become unstable and fuse forming multilamellar vesicles again.

The next step is to place a droplet of this vesicle solution on a freshly cleaved mica surface. Normally, a longer incubation time would yield better results. The membrane surface are often covered by excess vesicles and other debris. They are easily washed away by a moderate salt solution, such as 150 
$\mathrm{mM} \mathrm{NaCl}$. Finally, the sample was shortly rinsed with the buffer in which multilamellar suspension was prepared.

\subsubsection{Sample preparation for DSC and ultrasonic spec- trometry experiments}

Differential scanning calorimetry and ultrasound spectrometry experiments were performed on different concentrations of multilamellar and large unilamellar lipid vesicle (LUV) suspensions. LUVs were obtained using a smallvolume extrusion apparatus (MacDonald et al., 1991) provided by Avestin (Ottawa, Canada). The multilamellar vesicles were extruded through polycarbonate filters with $100 \mathrm{~nm}$ pores size mounted in the mini-extruder (see Figure 2.9) and fitted with two $1.0 \mathrm{ml}$ syringes. Usually we subjected samples to 21 times pass through the filter membrane. An odd number of passages was performed to avoid contamination of the sample by multilamellar vesicles which might not have passed through the filter. During the extrusion process the temperature of the sample was kept above the melting point of the lipids that makes it easier the pushing of the lipid suspension through the filter. For that the whole extruder was enclosed in a metal shield with circulating water of the required temperature. Right before the filling of the calorimeter or of the resonator the solution of extruded vesicles was degassed for 15 minutes. 


\section{Chapter 3}

\section{Results}

\subsection{Supported lipid membranes}

The ability of lipid bilayers to establish stable films on a hydrophilic support gives researchers a possibility to visualize and to do surface analysis of these model membranes using atomic force microscopy.

In the topographic AFM images the topology of the lipid membrane appears as light brown to dark brown tones. On this scale, dark brown colors correspond to lower lying regions and light brown colors correspond to higher lying regions of the membrane. Figure 3.1 presents an AFM image of a DPPC lipid membrane. In this particular image one can see different possible situations of the lipid membranes fusion on mica. Very dark regions on the image correspond to the mica support - that parts of the mica which were not covered by a lipid bilayer because of too short incubation time or not high enough lipid concentration (see section 2.2 .3 on page 53 ). In very bright colors appear pieces, or "islands", of the second lipid bilayer which lies on the top of the first bilayer. The last one is in direct contact with the mica surface and covers almost all image area. The height of the first DPPC bilayer is between $5-6 \mathrm{~nm}$ (measured from the mica support to the top of the bilayer) which is in accordance with other studies (Tamm and McConnell, 1985; Mou et al., 1994; Kaasgaard, 2003). The thickness of the second bilayer in the 
a

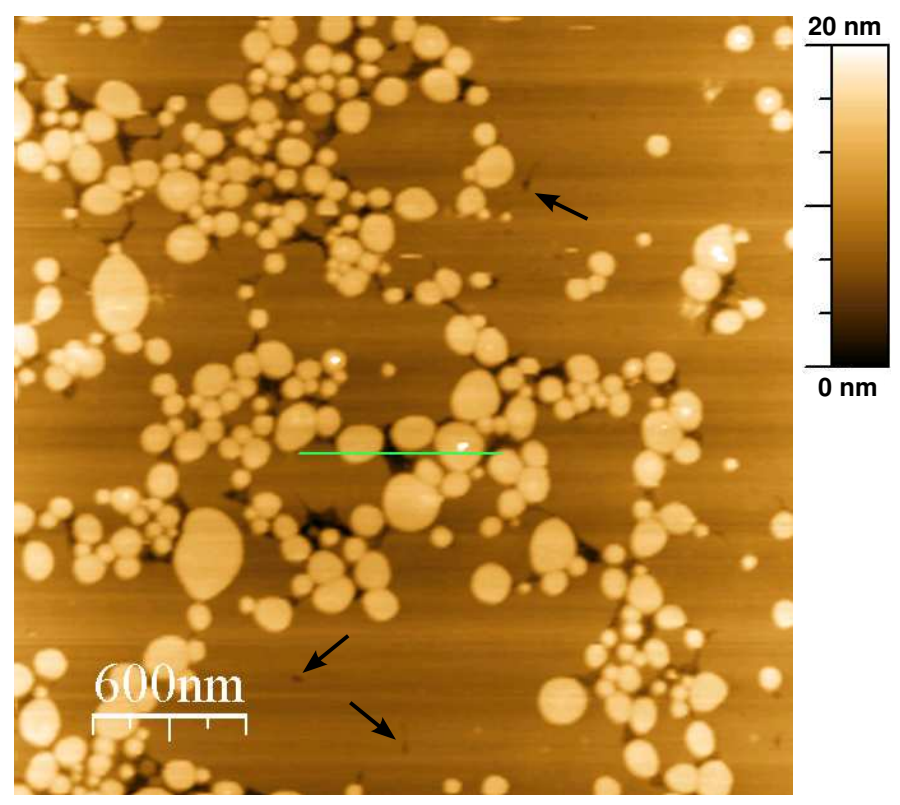

b

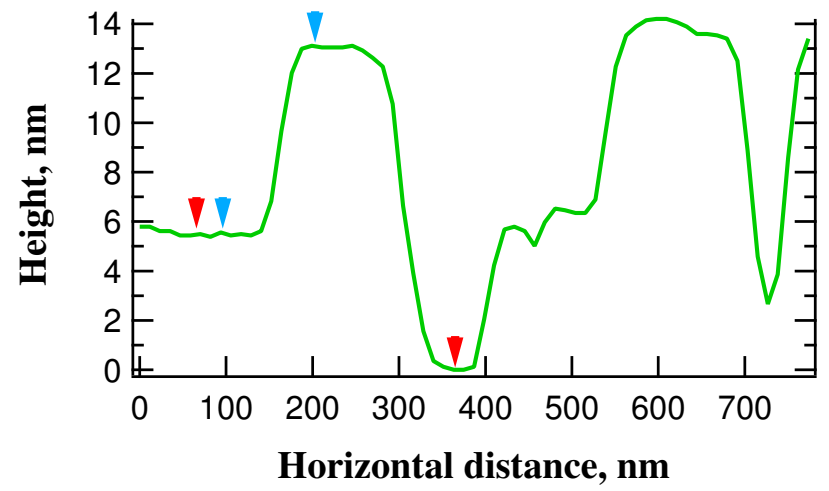

Figure 3.1: DPPC bilayers supported on mica. (a): $3 \times 3 \mu \mathrm{m}$ scan size height image produced by contact mode AFM. Black arrows point to membrane defects. (b): cross-section plot along the green line on the height image, from which thicknesses of the first (the height between red markers) and the second bilayers (the height between blue markers) are $\sim 6 \mathrm{~nm}$ and $\sim 8 \mathrm{~nm}$ respectively.

height profile (see Figure 3.1b) is about $2 \mathrm{~nm}$ bigger than the height of the first bilayer. This difference is caused by the presence of the layer of water 
a

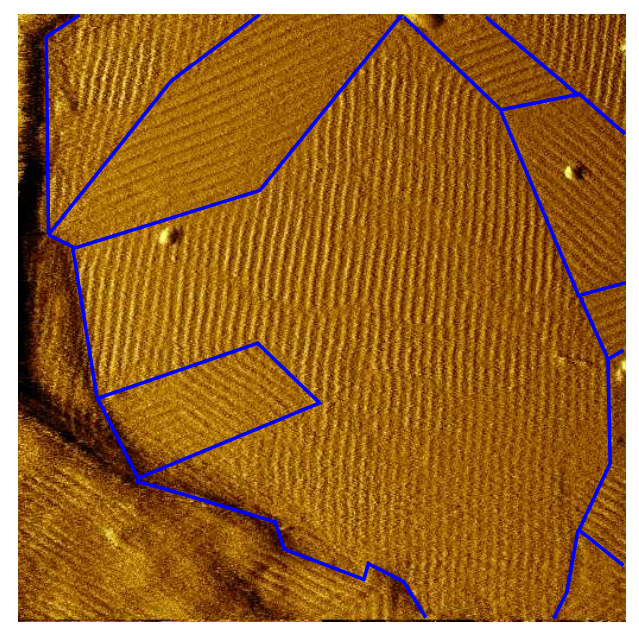

b

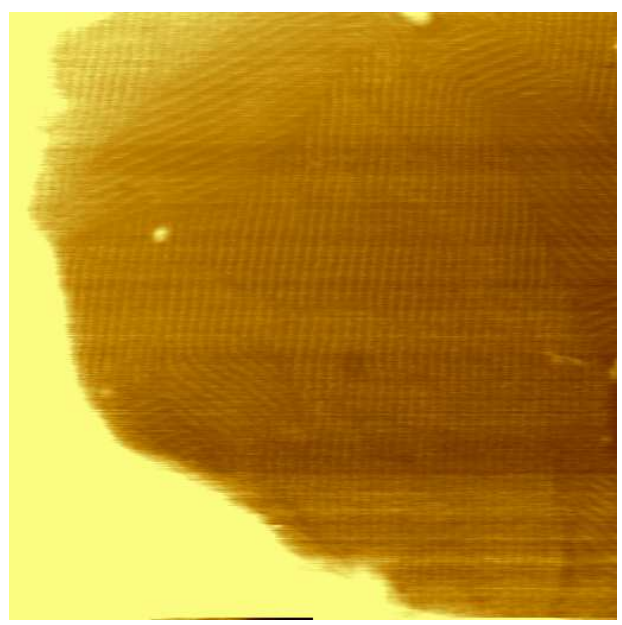

Figure 3.2: DPPC bilayer in ripple phase. (a): deflection image and (b): height image of $2 \times 2 \mu \mathrm{m}$ scan. The deflection image does not give any height information of the bilayer surface but serves for better detection of the membrane lateral structure. The domains of different ripple orientations have been outlined in (a).

between the bilayers (Nagle, 1980; Makowski and Li, 1984).

In general, mica-supported lipid bilayers in gel or fluid phases display a very flat surface that can contain some defects caused by defects in crystal structure of the mica, by contamination or partially fused lipid bilayers. Lipid bilayers in ripple phase appear in AFM images in a completely different fashion. In this state, which exists in a temperature range between the pretransition temperature and the main phase transition temperature, the lipid bilayer surface is characterized by corrugations in the lipid bilayer with a well-defined periodicity (see Figure 3.2). On this image the visualized structure of ripple phases are periodic linear arrays of ripples, which at certain points change their direction by characteristic angles of $60^{\circ}$ or $120^{\circ}$. The periodicity of ripples was determined to be equal to $\sim 30 \mathrm{~nm}$ that is in good agreement with other studies (Tenchov et al., 1989; Heimburg, 2000; Leidy et al., 2002).

The application of Tapping mode in liquids (see section 2.1.1 on page 41), 
a

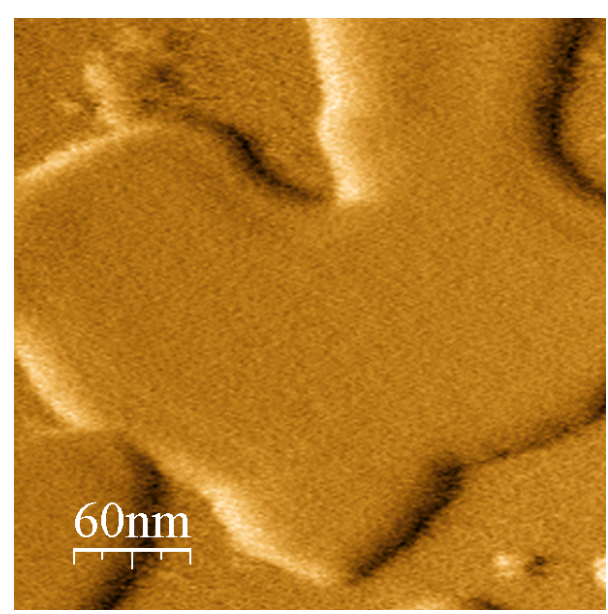

b

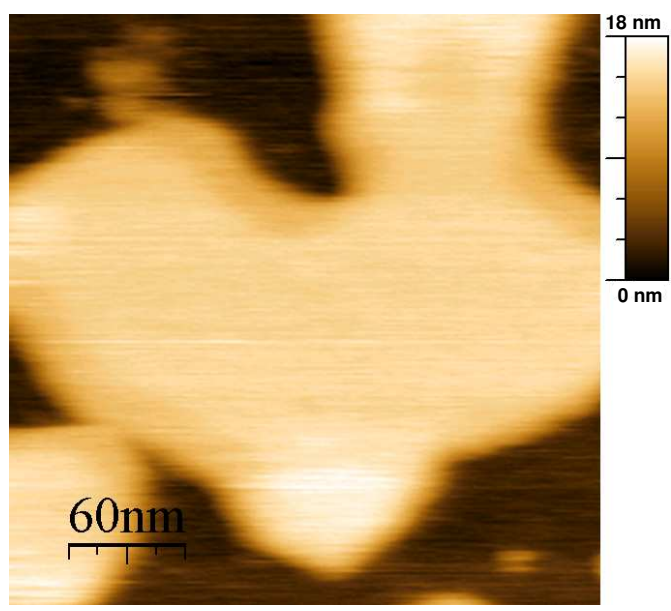

Figure 3.3: Tapping mode images of a DMPC membrane. (a): deflection and (b): height images of $300 \times 300 \mathrm{~nm}$ scan.

which is a more delicate AFM method for imaging lipid bilayers than Contact mode (see section 2.1.1 on page 39), was successful with DMPC membranes down to scan sizes of several hundreds of nanometers (see Figure 3.3). To reach such a high resolution working in Contact mode in liquids for lipid bilayers was near impossible. Another advantage of the Tapping mode, as was mentioned previously, is the possibility to do phase imaging simultaneously with collecting the topographical information of the sample surface (see section 2.1 .1 on page 43$)$.

In Figure 3.4 height and phase images of a supported DLPC lipid bilayer are represented. They look similar but provide additional information about completely different aspects of the sample surface properties. While with height images (see Figure 3.4a) one determines the topography of the DLPC lipid bilayer and one can estimate the XY-distances and the Z-height, the phase image (see Figure 3.4b) of exactly the same area gives the possibility of a qualitative estimation of viscoelastic properties of the sample surface. On the cross-section phase plot (see Figure 3.4d) one can clearly see that the lipid bilayer effects a noticeable contribution to the phase shift to AFM 
a

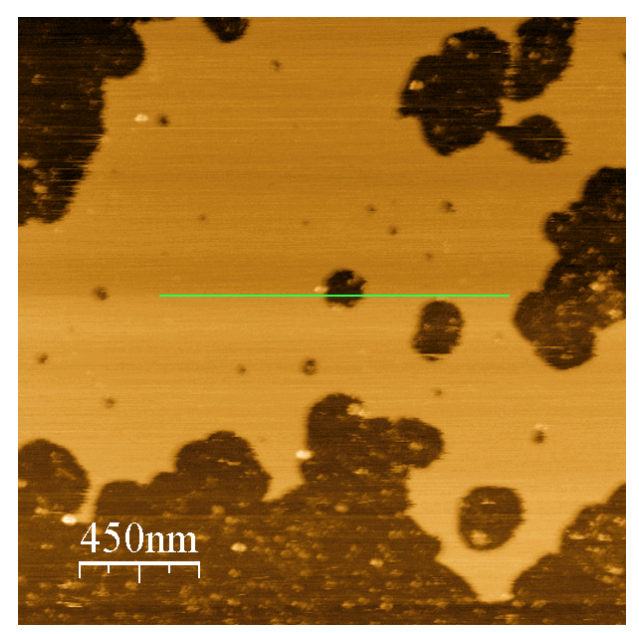

C

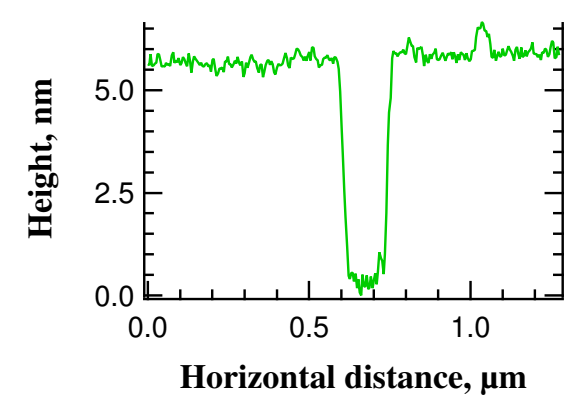

b

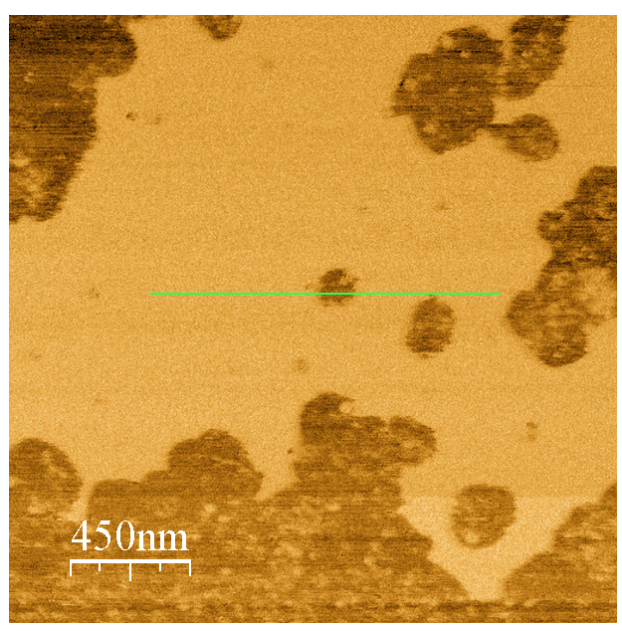

d

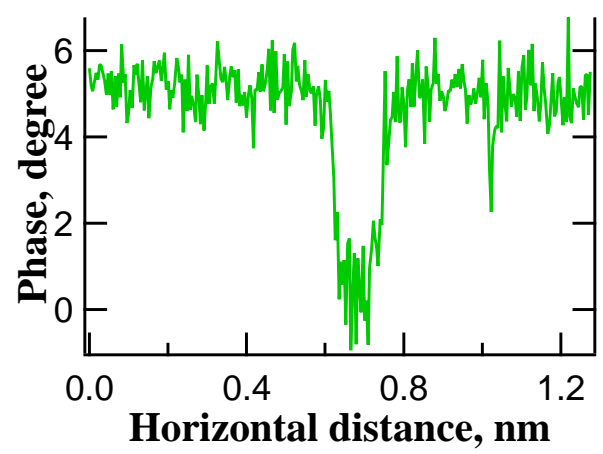

Figure 3.4: Tapping mode images of a DLPC membrane. (a): height and (b): phase images of $2.2 \times 2.2 \mu \mathrm{m}$ scan. (c) and (d) are cross-section profile and phase plot respectively made along the green lines representing information from the same scan line and the same position on the sample surface.

tip vibrations in comparison with the mica surface. This could be explained in terms of the sample elasticity. Since the DLPC lipid bilayer is obviously softer than the mica support and therefore the AFM tip can penetrate deeper into and transfer greater amounts of mechanical energy to the sample. This is reflected in the greater phase shift. 


\subsection{Domain formation in two-component lipid membranes.}

Using atomic force microscopy we have imaged lipid bilayers supported on a mica surface of the same DLPC:DPPC 33:67 ratio as in confocal fluorescence microscopy experiments of Hac et al. (2005) mentioned in chapter 1 on page 19. The typical AFM height image for those two-component membranes at $22^{\circ} \mathrm{C}$ is shown in Figure 3.5a. Deep black regions in the image correspond to the mica surface and the elongated bright white region at the center is a result of a second lipid bilayer which lies on top of the first. As it can be clearly seen, the first bilayer demonstrates the coexistence of at least three sorts of lipid domains with different height scales (further in the text - I, II and III lipid domains). From the cross-section profile along the green line in Figure 3.5b the height difference between the I lipid domain and the II lipid domain is approximately $\sim 0.9 \mathrm{~nm}$, and between the II lipid domain and the III lipid domain is about $\sim 0.6 \mathrm{~nm}$. Mainly the continuous II lipid domain with the lateral dimensions in the micrometer range can be found in the bilayer, whereas the I lipid domains and III lipid domains are embedded and randomly distributed within the plane of the II lipid domain. II and III lipid domains have characteristic sizes between tens to hundreds of nanometers. The background temperature was controlled by varying a room temperature using airconditioner, and the temperature was measured by a thin thermocouple at the sample stage at a position in close contact with the supported lipid bilayers.

We have also applied the phase imaging technique to this sample in order to check the viscoelastic properties of the detected lipid domains. The AFM scan of the left neighboring region relatively to the one presented in the Figure 3.5, made without withdrawing of the tip, is shown in Figure 3.6. From the phase image (Figure 3.6b), which was collected simultaneously with the topography image (Figure 3.6a), one can see that the mica surface at the center and at the top left corner of the image appears in deep black 
a

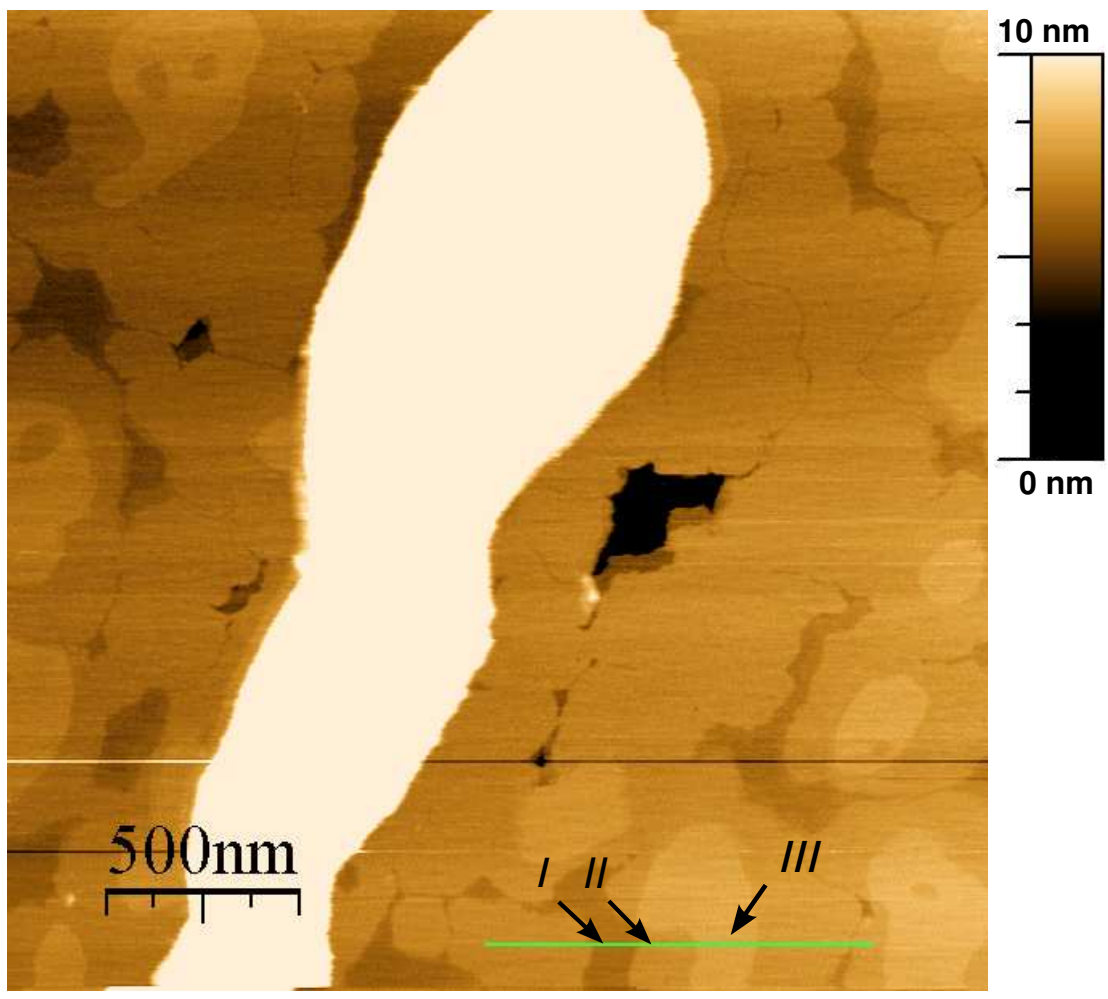

b

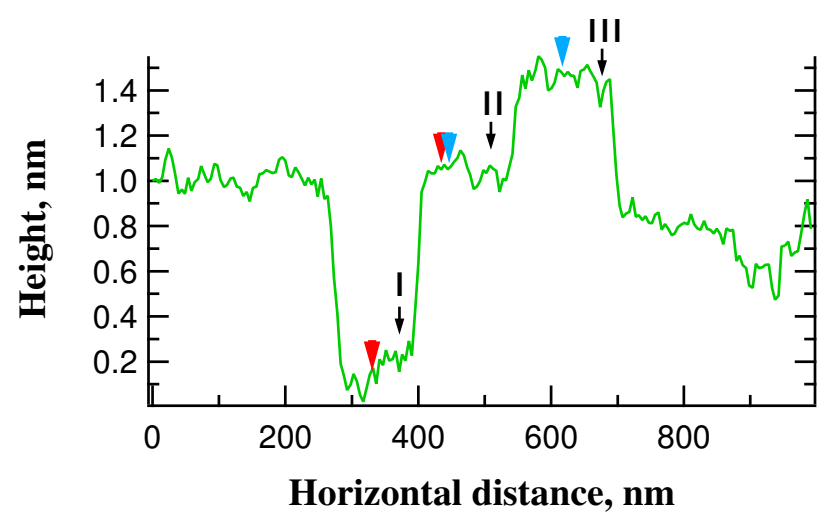

Figure 3.5: AFM 2.5 $\times 2.5 \mu \mathrm{m}$ image of DLPC:DPPC 33:67 membrane at $22^{\circ} \mathrm{C}$. (a): height image and (b): cross-section plot along the green line, where the height of $\sim 0.9 \mathrm{~nm}$ between red markers corresponds to the height difference between $I$ and II lipid domains, and the height of $\sim 0.6 \mathrm{~nm}$ between blue markers corresponds to the height difference between II and III lipid domains. 
a

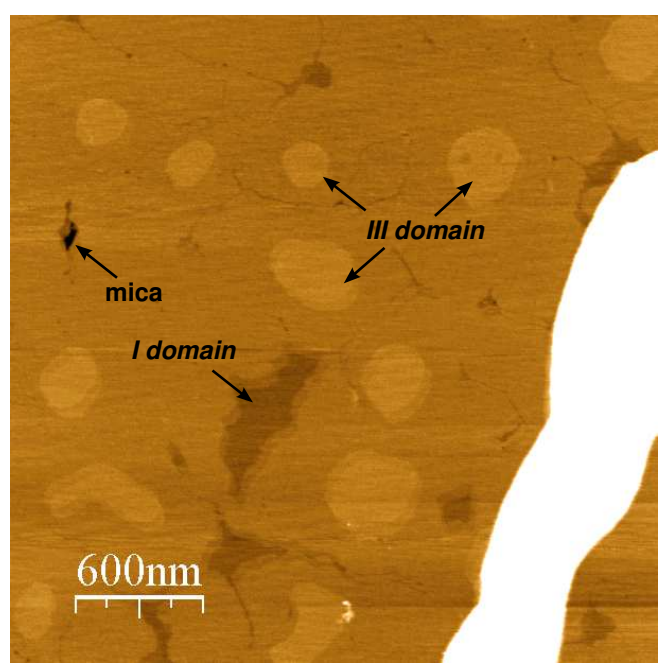

b

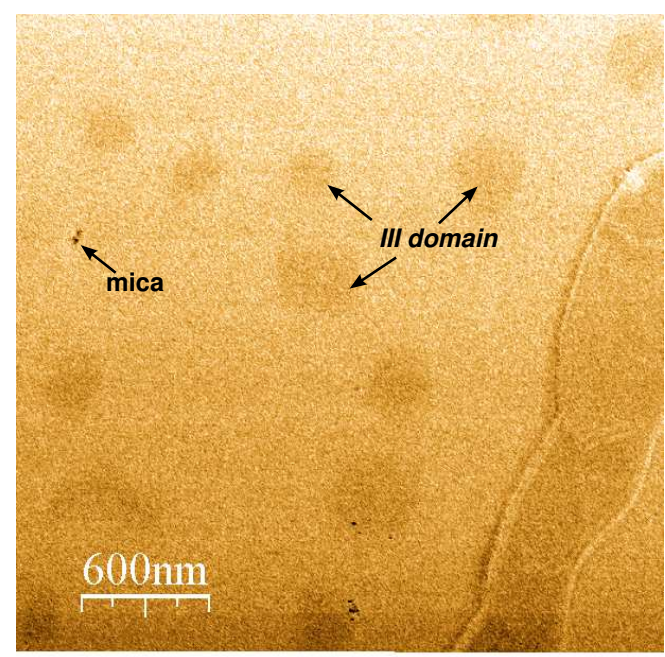

Figure 3.6: (a) Height and (b) phase images of 33:67 DLPC:DPPC lipid bilayer $(3 \times 3 \mu \mathrm{m}$ scan $)$. Different color tones in phase images indicate the phase variations of the oscillating tip that is in turn believed to be caused by variations of the bilayer stiffness.

color. Whereas the surrounding lipid bilayer has a much brighter color which means that the phase shift of the tip oscillations tapping the mica surface was much smaller in comparison with the bilayer surface. This happens because mica has a greater stiffness than the lipid membrane (see section 2.1.1 on page 43). However, the most important point in the phase map of the shown DLPC:DPPC membrane is that the III lipid domains induced an observable phase shift while the I lipid domains and II lipid domains were indistinguishable from one another in phase contrast. This means that the lipid domains, which have the greatest thickness in the topography image (III lipid domains), are stiffer than the two others that have two smaller height scales (I lipid domains and II lipid domains). The elastic properties of I lipid domains and II lipid domains are the same or the difference is too small to be detected with the used AFM method. 


\subsection{Peptide-containing lipid membranes}

\subsubsection{DSC experiments}

Excess heat capacity profiles (further in the text called " $c_{P}$-profiles") of DMPC and DPPC lipid vesicle suspensions with different molar concentrations of alamethicin and melittin, obtained using the differential scanning calorimetry method, are displayed in Figure 3.7. For a better data representation plots were shifted along the heat capacity axis for a constant offset.

The heat capacity curve of the pure DMPC vesicles in Figure 3.7a displays a main phase transition at $23.7^{\circ} \mathrm{C}$. The shape of the transition peak and the main phase transition temperature, $T_{m}$, are affected by increasing concentrations of the melittin as seen from the values listed in Table 3.1. With the highest measured peptide concentration of $2 \mathrm{~mol} \%$ the phase transiton temperature is decreased by only $\sim 1^{\circ} \mathrm{C}$. The melting enthalpy, $\Delta H$, which is determined as the area under the $c_{P}$-curves in Figure 3.7, is listed in Table 3.1. It is noted that the transition enthalpy decreases very slightly when the concentration of the melittin in the vesicles increases. These changes in the enthalpy are on the order of systematic error. In the case of DPPC vesicles (see Figure 3.7b), with the increase of melittin concentration the observed shift to low tempretures of the phase transition temperature is smaller in comparison to DMPC suspensions. In both cases of melittin containing lipid vesicles, the decrease in the lipid phase transition temperature caused by increasing concentrations of the peptide was accompanied by the appearance of a low-temperature shoulder on the transition peak.

A similar behavior is demonstrated by alamethicin containing lipid membranes (see Figure 3.7c and Figure 3.7d) with a slight shift to low temperatures, but with a smaller broadening of the lefthand slope in the peak than in the case of melittin. As it can be seen, the heat capacity profiles of fluid-togel transitions in DMPC and DPPC membranes for both peptides, melittin and alamethicin, have a similar tendency - slight shifts of the transition peak to lower temperatures with the increase of peptide concentration accompa- 


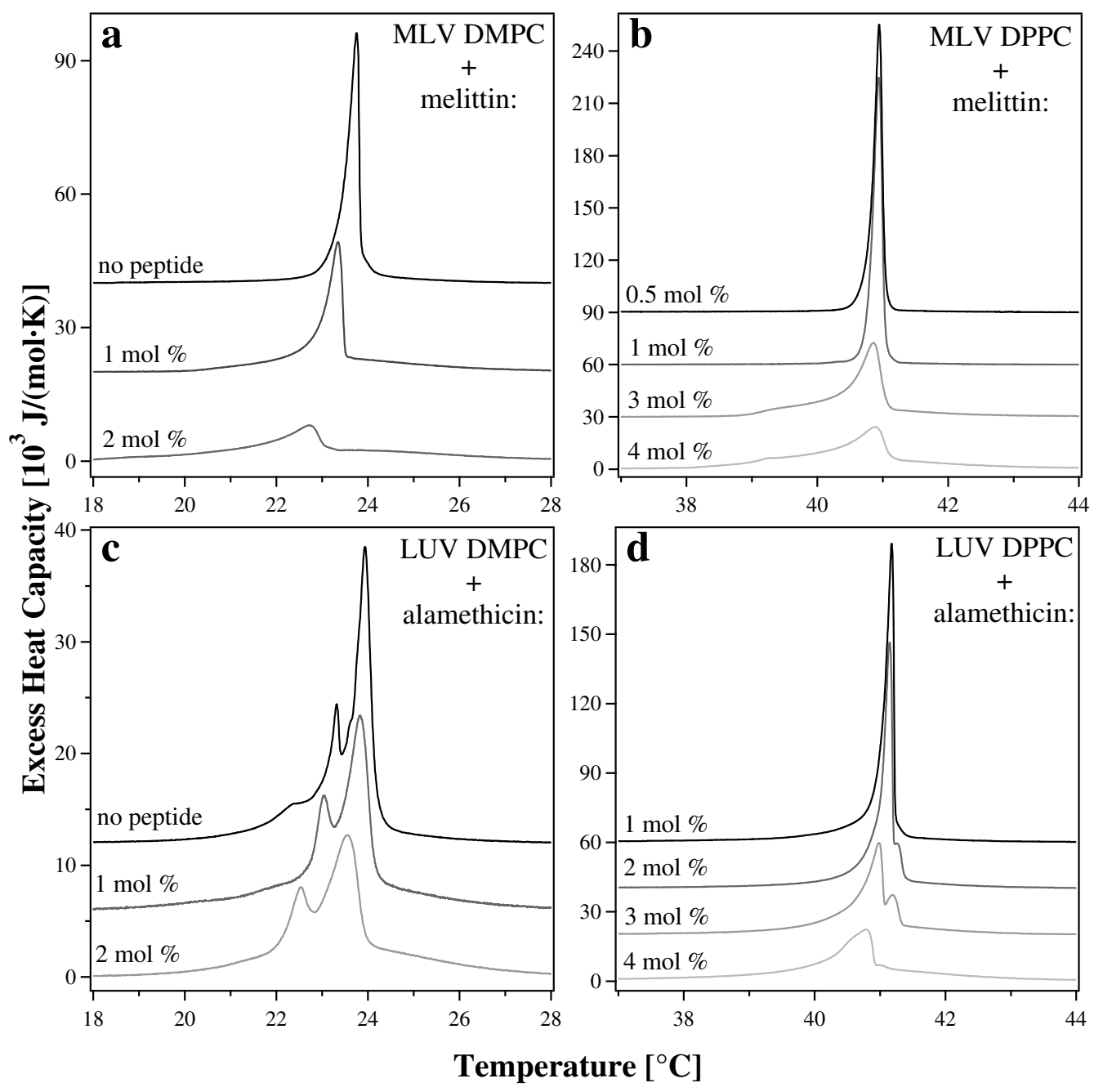

Figure 3.7: (a) and (b): $c_{P}$-profiles of DMPC and DPPC extruded vesicle suspensions (LUV - large unilamellar vesicles) with different alamethicin content; (c) and (d): $c_{P}$-profiles of DMPC and DPPC multilamellar vesicle suspensions (MLV) with different melittin concentrations. For a better data representation the curves were vertically shifted by a constant value. The melting temperatures $T_{m}$ and enthalpy changes $\Delta H$ can be seen in Table 3.1.

nied by assymetric broadening. The melting enthalpy in all four cases are not affected considerably. The deviations are on the order of the systematic error.

In $c_{P}$-profiles of DPPC suspensions with alamethicin added (see Fig- 
Table 3.1: Melting temperatures $T_{m}$ and enthalpy changes $\Delta H$ determined from $c_{P}$-profiles shown in Figure 3.7.

\begin{tabular}{|c|cc|cc|ccc|cc|}
\hline \multirow{2}{*}{} & \multicolumn{3}{|c|}{ Alamethicin } & \multicolumn{4}{c|}{ Melittin } \\
\cline { 2 - 10 } & \multicolumn{2}{|c|}{ DPPC } & \multicolumn{2}{c|}{ DMPC } & \multicolumn{2}{c|}{ DPPC } & \multicolumn{2}{c|}{ DMPC } \\
\cline { 2 - 10 } & $T_{m}$ & $\Delta H$ & $T_{m}$ & $\Delta H$ & $T_{m}$ & $\Delta H$ & $T_{m}$ & $\Delta H$ \\
\hline $0.0 \mathrm{~mol} \%$ & - & - & 23.6 & 22.2 & - & - & 23.7 & 25.9 \\
\hline $0.5 \mathrm{~mol} \%$ & - & - & - & - & 41.04 & 33.6 & - & - \\
\hline $1.0 \mathrm{~mol} \%$ & 41.18 & 34.8 & 23.4 & 22.5 & 40.94 & 35.9 & 23.3 & 25.2 \\
\hline $2.0 \mathrm{~mol} \%$ & 41.17 & 34.6 & 22.9 & 23.1 & - & - & 22.7 & 22.5 \\
\hline $3.0 \mathrm{~mol} \%$ & 40.98 & 29.7 & - & - & 40.86 & 34.4 & - & - \\
\hline $4.0 \mathrm{~mol} \%$ & 40.79 & 32.4 & - & - & 40.89 & 35.5 & - & - \\
\hline
\end{tabular}

ure $3.7 \mathrm{~d}$ ) one can also observe that at $2 \mathrm{~mol} \%$ of alamethicin a small second peak appears at the high temperature slope of the main transition peak. It is more developed in the $c_{P}$-profile for $3 \mathrm{~mol} \%$ of peptide and almost disappears with further increasing of alamethicin content up to $4 \mathrm{~mol} \%$. The heat capacity profile of large unilamellar vesicle (extruded) suspensions of pure DPPC normally does not show up a splitting of the peak, but in the case of extruded DMPC vesicles this behavior takes place (see Figure 3.7c) and is not affected considerably by the presence of alamethicin. The splitting of the peak in $c_{P}$-profiles of pure DMPC suspensions is believed to be related to the changes of vesicle geometry in the lipid melting regime, by analogy to a transition between lipid vesicles and a bilayer network during lipid phase transition of DMPG dispersions detected in electron microscopy experiments (Schneider et al., 1999).

\subsubsection{Ultrasonic sound velociy experiments}

In Figure 3.8, the heat capacity $c_{\mathrm{P}}$ and sound velocity number $u$ are displayed as a function of temperature for suspensions of large unilamellar vesicles from DMPC (Figure 3.8, left panel) and DPPC (Figure 3.8 right panel) with 


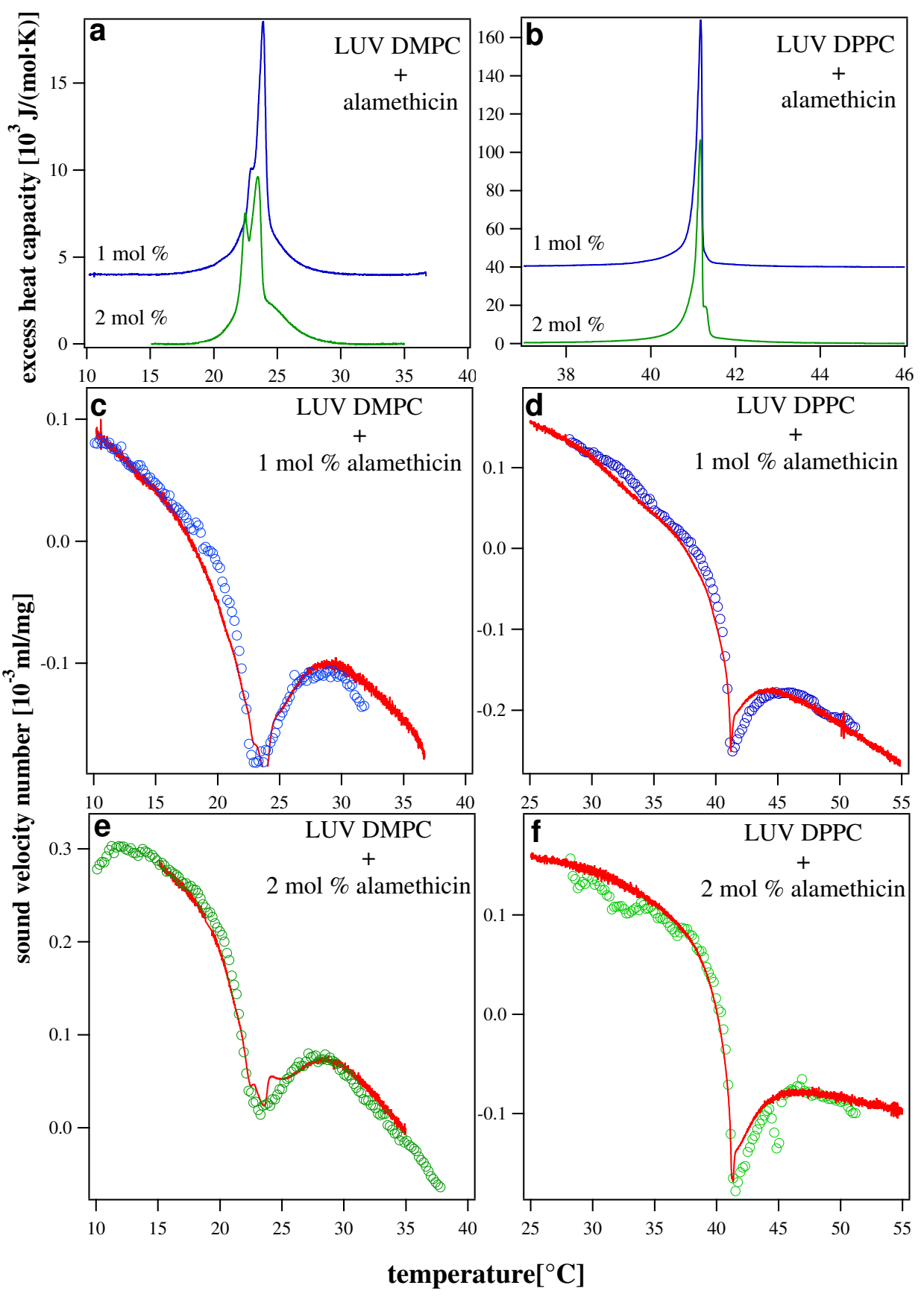

Figure 3.8: $c_{P}$-profiles (top), experimental ultrasonic velocity number and from the heat capacity recalculated ultrasonic velocity number (red solid curves) for DMPC (left panel) and DPPC (right panel) extruded vesicle solutions containing $1 \mathrm{~mol} \mathrm{\%} \mathrm{(middle)} \mathrm{and} 2 \mathrm{~mol} \%$ (bottom) of alamethicin. 


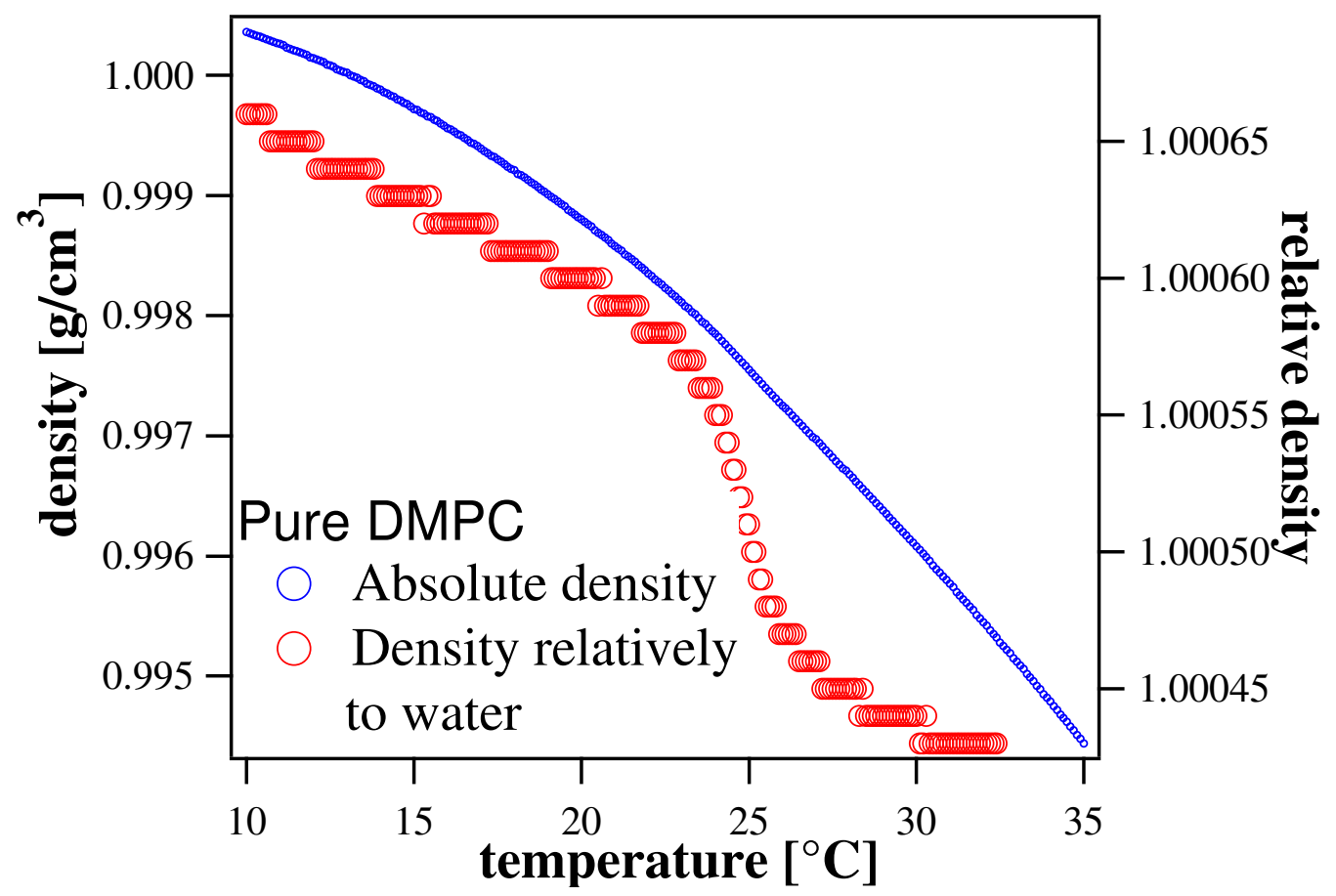

Figure 3.9: Density of a $2.5 \mathrm{mM}$ DMPC lipid suspension measured with a highprecision densitometer. The density smoothly decreases in the melting regime and changes are on the order of $\sim 0.1 \%$. In the density relative to water one can see a characteristic step-wise change of the density of the lipid water suspension during the phase transition.

alamethicin. Each set of figures shows, around the gel/fluid phase transition temperature $T_{m}$ of the lipid systems, the experimental $c_{\mathrm{P}}$ scans (top), the measured sound velocity numbers $u$ (plotted as circles), as well as the $u$ data, which, according to the theoretical model mentioned in section 1 on page 32 , results from the $c_{\mathrm{P}}$ data, for $1 \mathrm{~mol} \%$ (middle) and $2 \mathrm{~mol} \%$ (bottom) of peptide (plotted as solid red curve). Velocity number curves demonstrate a minimum typical for lipid suspensions of the sound velocity at the melting temperature (velocity number $u$ reflects the absolute change of the sound velocity of the sample solution relatively to the reference, see eq. 1.12 on page 35). Since the density of lipid suspensions normally smoothly decreases in the melting regime and changes are on the order of $\sim 0.1 \%$ (see Figure 3.9), such minimum of the sound velocity reflects a maximum in the adiabatic 
compressibility (see eq. 1.11 on page 35 ).

The increase of alamethicin concentration in DMPC vesicles from 1 to $2 \mathrm{~mol} \%$ (Figure 3.8c and Figure 3.8e) causes the reduction of the amplitude in the sound velocity minimum accompanied by a slight broadening. Such behavior of sound velocity number curves is in nice agreement with the influence of alamethicin on the temperature dependent heat capacity profiles obtained in calorimetric experiments for these samples (Figure 3.8a). In the case of DPPC suspensions containing alamethcin, a similar agreement of experimental $c_{\mathrm{P}}$-profiles and measured velocity number curves is clearly observed. Comparing the velocity number curves recalculated from heat capacity (red solid line), their close correlation becomes obvious.

\subsubsection{AFM experiments}

In Figure 3.10 typical AFM topography images of DPPC bilayers containing $1 \mathrm{~mol} \%$ of alamethicin are presented. As one can see from the $5 \times 5 \mu \mathrm{m}$ scan (Figure 3.10b) the surface of the supported bilayer is evenly perturbed by transmembrane defects which are believed to be peptide pores. A smaller scan size image $(2 \times 2 \mu \mathrm{m})$ at the nearby region of the same sample was made for a more detailed observation (see Figure 3.10a). A majority of alamethicin induced pores appear preferentially as round shaped holes with sizes in a range of $30-100 \mathrm{~nm}$, but there are some elongated pores with smooth round kinks. One can also note that mainly all pores are surrounded by some sort of shells which have a smaller height (darker color in the height image) in comparison with the overall bilayer.

From the section profile across one of the pores shown in Figure 3.10c we found that the height of the shell is in the range of $3-4 \mathrm{~nm}$ whereas the bilayer thickness was found to be $5-6 \mathrm{~nm}$. This bilayer height is in good agreement with literature values of the thickness of the gel phase DPPC lipid bilayer (Tamm and McConnell, 1985; Mou et al., 1994; Kaasgaard, 2003). The melting temperature of DPPC lipids is around $41^{\circ} \mathrm{C}$ and at room temperature (the used AFM microscope did not have a temperature control) 
a

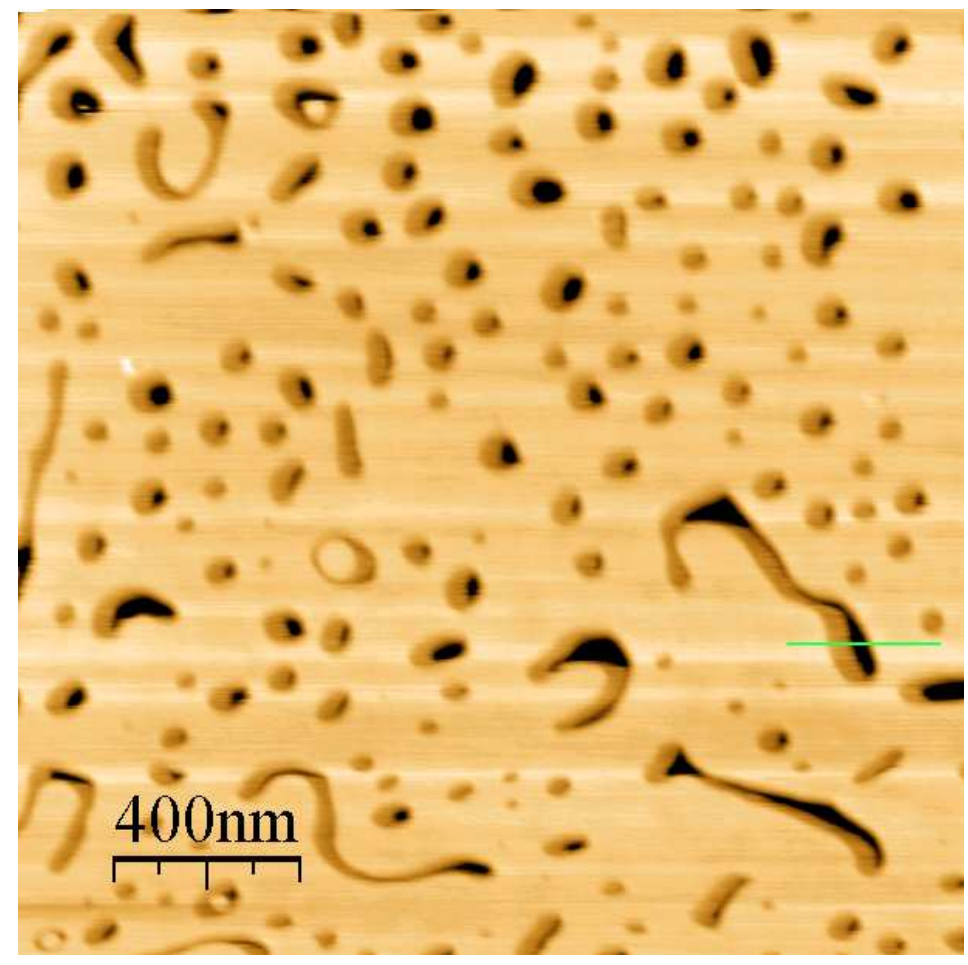

b

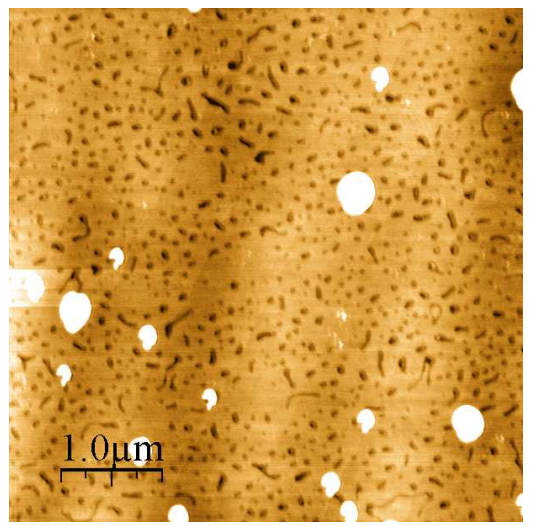

C

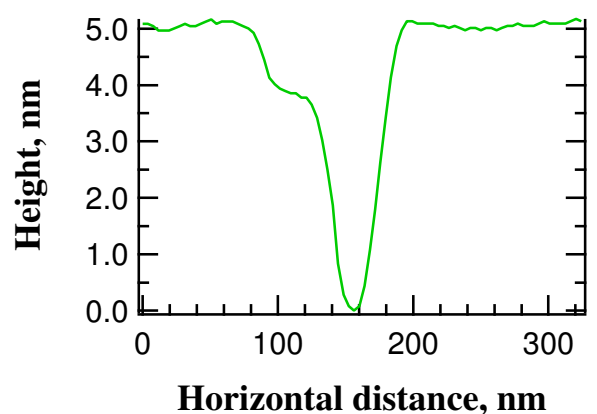

Figure 3.10: Contact mode height images of a DPPC lipid membrane with $1 \mathrm{~mol} \%$ of alamethicin. (b): Panoramic $5 \times 5 \mu \mathrm{m}$ scan. (a): $2 \times 2 \mu \mathrm{m}$ scan where the green line marks the cross-section shown in (c). Black arrows point to the nanoscopic lipid domains surrounding peptide pores. 
they are in gel phase. The height of the shells surrounding the alamethicin pores corresponds to the thickness of fluid DPPC membranes (Heimburg, 1998). Thus, we can assume that these shells are nanoscopic lipid domains in the fluid phase.

Figure 3.11 shows AFM images recorded from a DPPC lipid membrane with $4 \mathrm{~mol} \%$ alamethicin. A four times higher peptide concentration has a big effect on the pore formation. From a $5 \times 5 \mu \mathrm{m}$ scan (Figure $3.11 \mathrm{~b}$ ) one can see that at this concentration alamethicin develops mostly pores with a long, branched and irregular shape. Remarkable is also the fact that for this peptide concentration the height depressions in close vicinity to alamethicin pores were also detected (Figure 3.11a) that demonstrates a striking similarity with the case of lower peptide concentration discussed above.

Another similarity between the images of DPPC bilayers containing 1 and $4 \mathrm{~mol} \%$ alamethicin is a manner in which lipid shells appear in AFM scans. In both cases lipid shells of smaller height did not form a continuous ring around a peptide pore. Especially in the case of DPPC bilayers containing $1 \mathrm{~mol} \%$ alamethicin, where height depressions appear only on one side of a pore. One can claim that such characteristic depression close to the edges of the sample surface can be a consequence of an imaging artifact caused by a broken AFM tip. In practice, light damages of the AFM probe during experiment sometimes lead to its splitting due to the too high contact force adjusted or to artifacts in the AFM tip manifacturing. Then, the imaging in contact mode with such splitted tip (often it is splitted to two splinters) produces in height images doubling of surface sharp features (like edges of the lipid bilayer), since such features are detected twice with both splinters.

Concerning shells observed in our experiments one can further speculate that the AFM tip was splitted in a way that one of the splinters was shorter than the other (see Figure 3.12). So, a bilayer edge was detected in the beginning with the longer splinter (this is a real height of the bilayer) and then by the shorter tip splinter that reflects in AFM image as a bilayer of smaller thickness. Then, the width of shells is approximately equal to the 


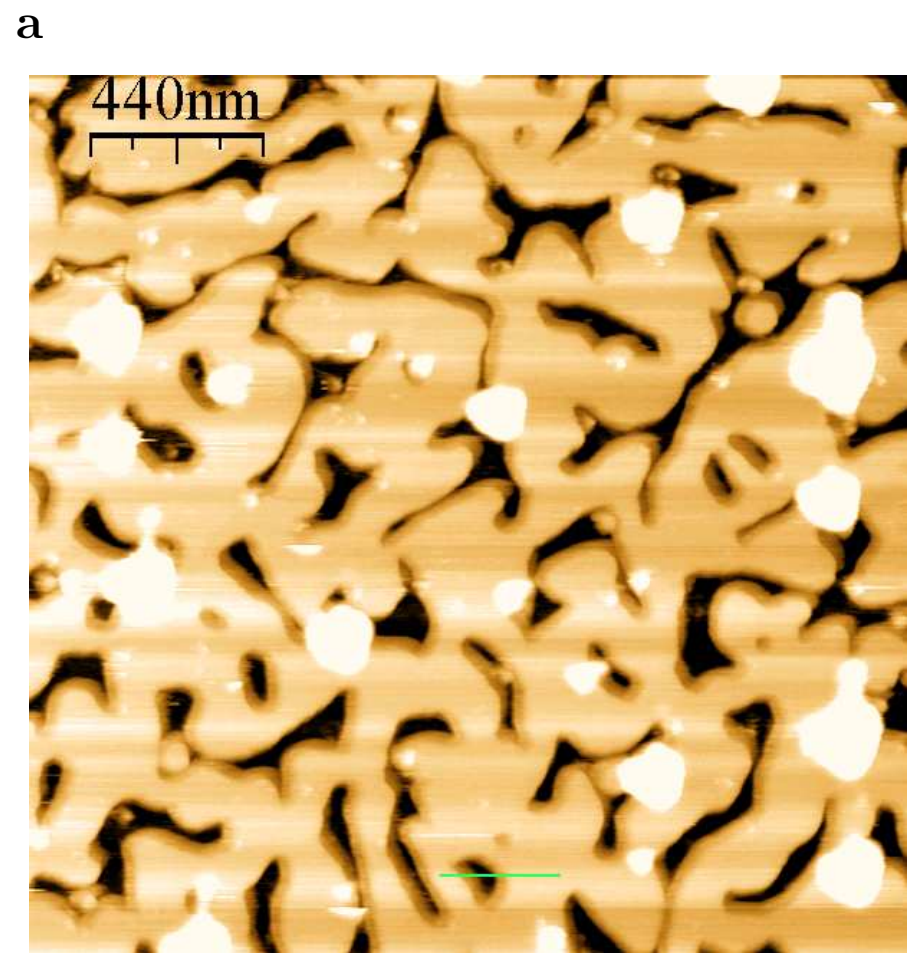

b

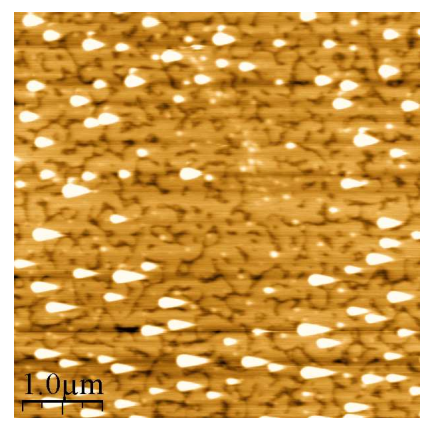

C

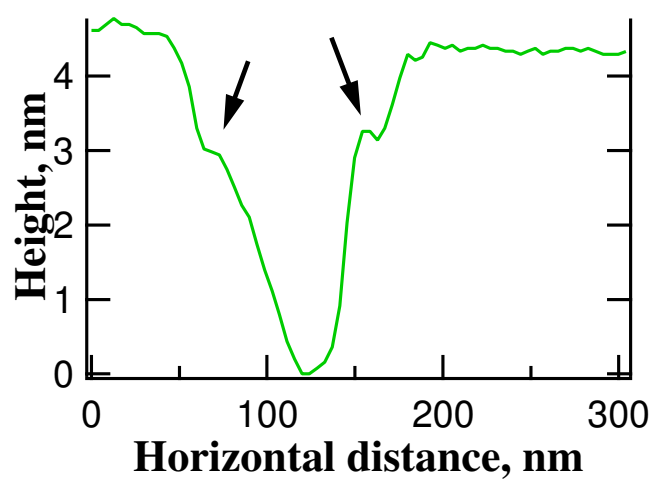

Figure 3.11: Contact mode AFM images of DPPC lipid membrane containing $4 \mathrm{~mol} \%$ alamethicin. (b): $5 \times 5 \mu \mathrm{m}$ topography image where alamethicin pores have an elongated and a branched shape. In a smaller scan size $(2.2 \times 2.2 \mu \mathrm{m})$ image (a) fluid lipid shells (in darker tones) around pores can be seen. Black areas correspond to the mica surface. (c) is a cross-section height profile along the green line extracted across one of the pores in (a). 


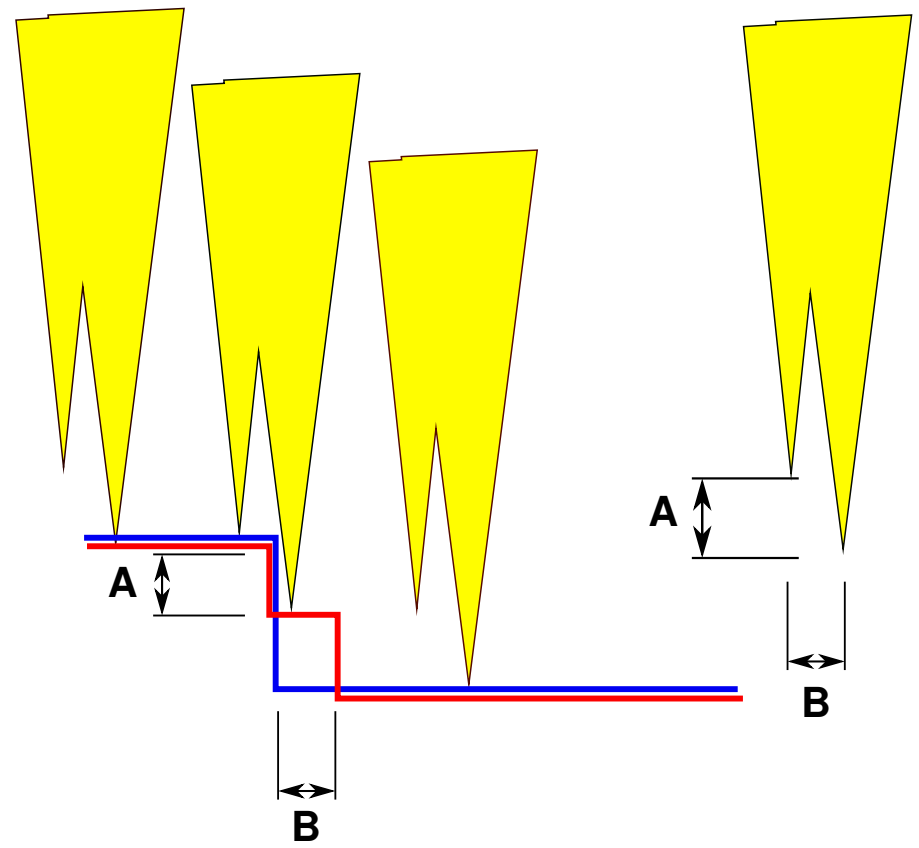

Figure 3.12: Schematic illustration of a broken AFM tip with asymmetric splitting. Blue line depicts a sample surface, red line is a height profile produced by the splitted AFM probe.

separation of two splinters and the height difference between thicker and thiner bilayers is caused by the different length of two splinters of the AFM tip.

Contradicting to the above discribed possible origin of observed shells it is necessary to note that a probability of the AFM tip splitting should be equal for all experiments with supported lipid bilayers. However, such height depressions at bilayers edge were observed by us only with peptide containing lipid membranes and never with pure membranes. We have experienced that, if the splitting of the tip happens, indeed this leads rather to the nearly synchronus doubling of surface features. In the presented height images of DPPC bilayer with $1 \mathrm{~mol} \%$ (Figure 3.10a) height depressions are detected preferentially from one side of transmembrane defects but they do not show up as an exact copy of higher edges. From the other side, observed shells in the image of DPPC bilayer with 4 mol \% (Figure 3.11a) do not demon- 
a

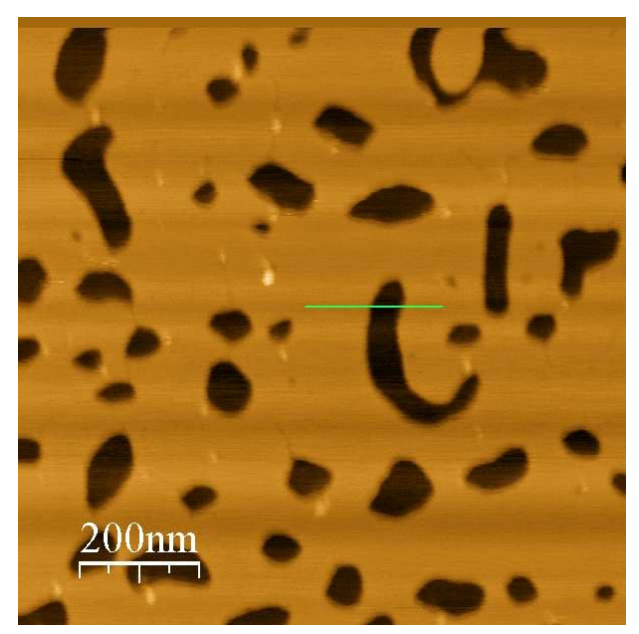

C

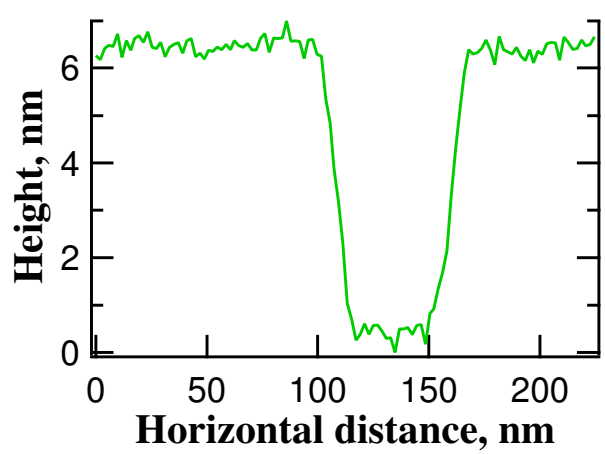

b

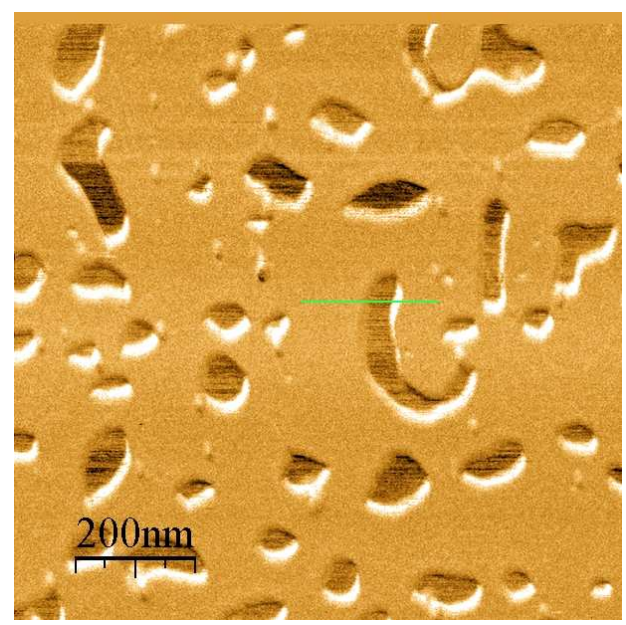

d

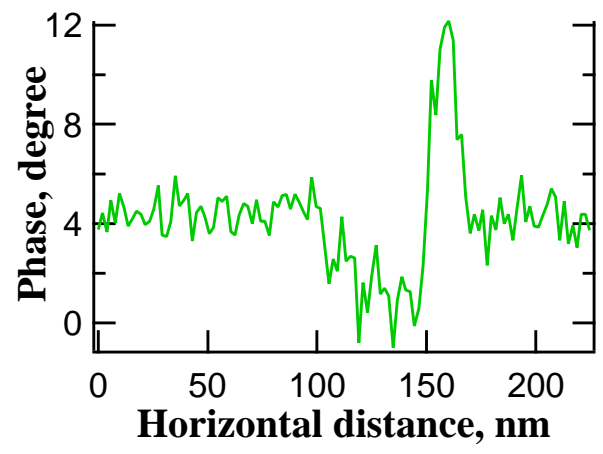

Figure 3.13: (a): Height and (b): phase images of a DPPC lipid membrane with $1 \mathrm{~mol} \%$ of alamethicin. The green line in both images depicts the cross-setion height (c) and phase (d) profiles.

strate any preferential orientation and even can be found in both sides of one particular pore as it shown in Figure 3.11c.

We also performed phase imaging of supported DPPC bilayers containing alamethicin in order to investigate the influence of pores formed by this peptide on the local compressibility of a lipid membrane. Topography and phase $1 \times 1 \mu \mathrm{m}$ images of a DPPC membrane containing $1 \mathrm{~mol} \%$ alamethicin are presented in Figure 3.13. Both images were simultaneously recorded 
operating AFM in the Tapping mode. This experiment has revealed that pores appear in a similar way, like it was shown above for the same peptide concentration (see Figure 3.10), but characteristic height depressions around the pores, which are meant to be fluid lipid domains, were not observed (see height profile in Figure 3.13c). On the other hand, in the phase image (Figure $3.13 \mathrm{~b}$ ) one can clearly see that regions close to the pore have a brighter contrast in comparison to the rest of the membrane (see also phase section profile across one of the pores in Figure 3.13d). This increase in phase delay of the AFM tip vibrations indicates that the probed surface close to the peptide pore is softer or, in other words, the local compressibility is higher. This peptide induced softening of lipids close to pores is in agreement with the mentioned Monte Carlo simulations where it was shown that lipids fluctuate higher between gel and fluid state nearby peptide clusters. Larger fluctuaions mean in particular larger compressibility. The absence of fluid lipid shells around pores, which were observed for lipid bilayers with the same alamethicin content, could be a consequence of slight temperature deviations between experiments since all presented AFM images were obtained at room conditions. It is possible to find soft regions without finding fluid rings, because the lipid surrounding the pore may still be mostly gel but still be within the transition.

In order to obtain structural information about melittin aggregation in gel and fluid lipid membranes we visualized DPPC and DLPC lipid bilayers (at room temperature in gel and in fluid phase, respectively) containing $1 \mathrm{~mol} \%$ melittin. As one can see from Figure 3.14a melittin establishes in gel lipid membrane a branched net of elongated transmembrane pores which are much more developed in comparison to alamethicin containing bilayers with the same peptide content. Because of the close spacing of pore sides and the limited radius of curvature of the AFM tip it was not possible to find out whether the presence of melittin causes the melting of surrounding lipids. Nanoscopic lipid domains, however, were detected at the ends of a pore where they become to be wider. At the top right corner of the Figure 3.14a 
a

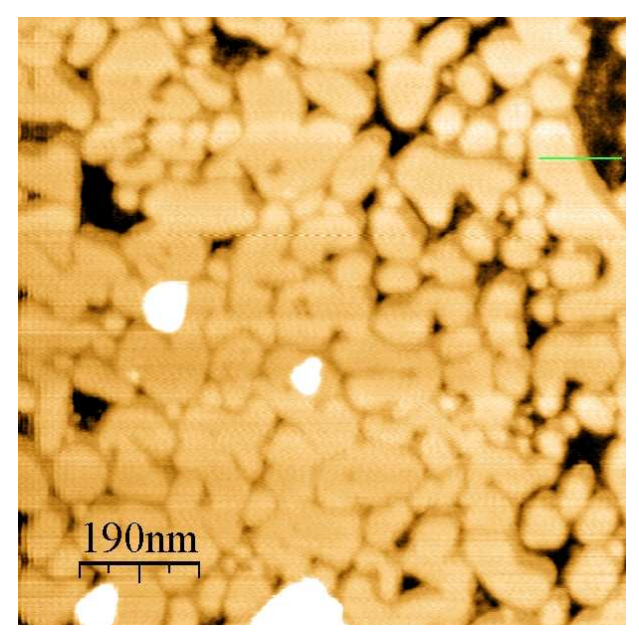

c

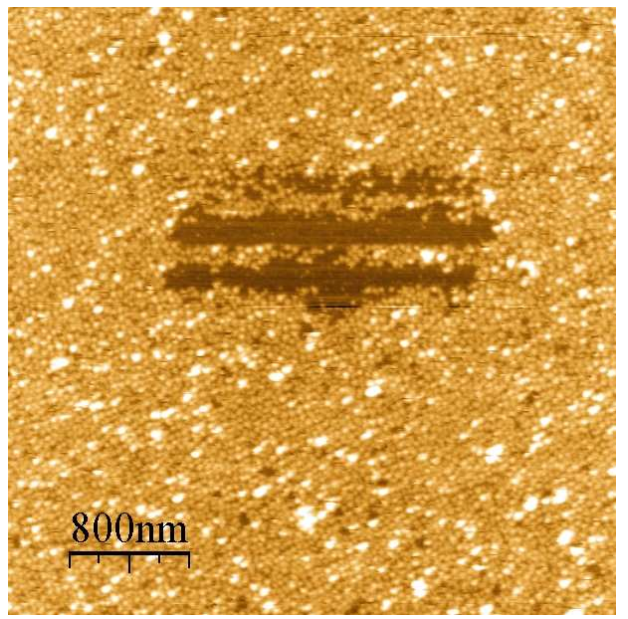

b

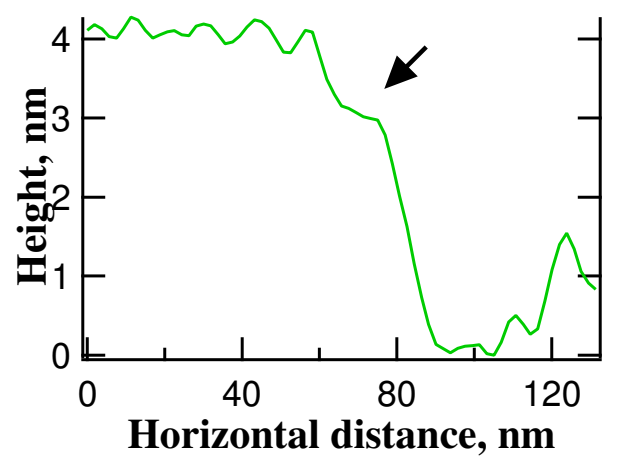

d

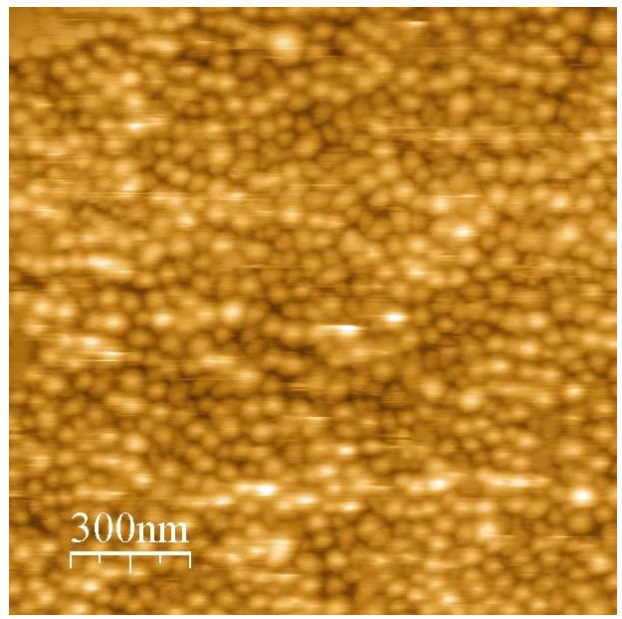

Figure 3.14: AFM images of DPPC and DLPC lipid membranes containing $1 \mathrm{~mol} \%$ of melittin.(a): $1 \times 1 \mu \mathrm{m}$ height image of DPPC bilayer, where green line marks a height profile shown in (a). Two height images of DLPC membranes with $4 \times 4 \mu \mathrm{m}$ and $1.5 \times 1.5 \mu \mathrm{m}$ scan sizes are shown in (c) and (d), respectively. At the center of the image in (c) the bilayer was artificially destroyed by scanning with a very high contact force and scan rate.

these domains can be also seen along open bilayer borders which do not enclose any "empty" space. Fluid lipid shells along those pieces of bilayer 
could be regarded as so-called inverted pores that melittin can create as reported previously (Faucon et al., 1995; Pott and Dufourc, 1995). Measured thicknesses of gel DPPC membranes and fluid lipid shells in the cross-section profile in Figure 3.14b differ from values normally observed for those objects which could be a consequence of a not perfectly adjusted contact force during the AFM experiment. This causes an insufficient quality of the image.

The pore formation by melittin in a fluid DLPC lipid membrane is strikingly different in comparison to gel DPPC membranes. As it can be seen in Figure 3.14c and Figure 3.14d, melittin forms a widespread net of transmembrane defects. DLPC membrane seems to be highly perturbed by transmembrane pores. Such a high density of melittin induced pores in DLPC bilayers at room temperature could be an explanation for the strong lytic power of this peptide in native biological membranes. 


\section{Chapter 4}

\section{Discussion and conclusions}

One of the aims of this study was the investigation of the domain and microdomain formation in artificial planar membranes on a nanoscopic scale, using Atomic Force Microscopy (AFM).

For years the investigation of membrane structure and composition has been of great interest. It has been established from experimental (Kusumi and Sako, 1996; Yang and Glaser, 1996; Bagatolli and Graton, 1999; Nielsen et al., 2000; Leidy et al., 2002; Tokumasu et al., 2003; Hac et al., 2005; Seeger et al., 2005) and theoretical (Jain and White, 1977; Mouritsen, 1991b) studies that the membrane structure is not homogeneous but contains various heterogeneities. The most attractive region in lipid phase diagrams of binary mixtures is that one corresponding to the coexistence of gel and fluid domains. Superposition of gel and fluid domains in the phase coexistence region on Giant Unilamellar Vesicles (GUVs) was observed. The direct observation and analysis of gel and fluid domains were possible by Confocal Fluorescence Microscopy (CFM). With this technique phases can be identified on the known partition behavior of a given fluorescent marker. With CFM, macroscopic domains of lipids in gel and in fluid phase can be visualized. Their shapes depend on the lipid composition and on the temperature of the measurement. In the case of DLPC:DPPC mixtures, the gel domains are presented on a vesicle surface as "dendritic" shaped structures with char- 
acteristic sizes in the range of micrometer (Hac et al., 2005; Seeger et al., 2005).

Besides CFM Atomic Force Microscopy makes it also possible to produce an image of domain formation. Nielsen et al. (2000) were able to detect domains in one-component DMPC lipid monolayers near the gel/fluid phase transition and in binary DMPC:DSPC mixture monolayers supported on mica. Imaging two component DLPC:DPPC lipid bilayers Tokumasu et al. (2003) have shown that, at different DLPC-to-DPPC ratios, AFM images demonstrate a mixed architecture of nanoscopic and microscopic domains. Their height analysis of membrane thickness measured from AFM scans has revealed two distinctive height scales which correspond to coexisting DLPCrich and DPPC-rich domains.

AFM topography images of DLPC:DPPC 33:67 membranes recorded by us at $22^{\circ} \mathrm{C}$ demonstrated the possibility of more complicated behavior of such bilayers. We observed the coexistence of at least three sorts of lipid domains with different height scales (for simplicity called I, II and III lipid domains). The height difference between the I lipid domain and the II lipid domain was measured to be equal to approximately $\sim 0.9 \mathrm{~nm}$, and between the II lipid domain and the III lipid domain it was about $\sim 0.6 \mathrm{~nm}$. Similarly to the results obtained by Tokumasu et al. (2003) for DLPC:DPPC mixtures, we were able to see the microorganization of macrodomains. But in our case, the nanoscopic I lipid domains and III lipid domains are embedded and randomly distributed within the plane of the mainly continuous II lipid domain with the spatial dimensions in the micrometer range. Furthermore, doing phase imaging of the domains in the DLPC:DPPC 33:67 membrane we were able to detect that the lipid domains, which have the greatest thickness in the topography image (III lipid domains), are stiffer than the two others that have two smaller height scales (I lipid domains and II lipid domains). Such a behavior can be explained assuming, that the lipid molecules in the different lipid domains are in different states. For instance, III lipid domains are more likely to consist of preferentially lipids in gel state whereas I lipid 
domains and II lipid domains are formed mainly by lipids in the fluid state. In favour of this assumption are such facts as that the lipid compressibility of bilayers in gel and fluid phase have different compressibilities and thicknesses (Heimburg, 1998; Schrader et al., 2002).

Hac et al. (2005) have performed Monte Carlo (MC) simulations of DMPC:DSPC mixtures which demonstrate similar phase diagrams to DLPC:DPPC and therefore they have assumed that both systems have a similar behavior. They have shown that in the phase coexistence region the MC snapshots, which give a qualitative representation of the lipid membrane spatial organization, contain small fluid domains within a larger gel matrix, or small gel domains embedded into a fluid matrix, and gel domains of one species within gel domains of the other species (See Figure 1.9 on page 19). The same for the fluid phase. Therefore, one can expect more than two scales in domains heights. Our present AFM images of DLPC:DPPC mixtures are in good agreement with their theoretical findings.

Another goal of this work was to study the aggregation of small antibiotic petides, like melittin and alamethicin, in model lipid membranes. We used three experimental techniques, such as Differential Scanning Calorimetry (DSC), Ultrasonic Velocimetry and Atomic Force Microscopy, in order to investigate different aspects of lipid-peptide interactions. Both alamethicin and melittin are extensively studied native peptides that interact with lipid bilayers, resulting in an alteration of the cell's membrane permeability. The peptides are thought to contribute to this process through the formation of ion channels in the cell membrane and/or by changing the activity of existing channels (Dempsey, 1990; Cafiso, 1994; Kourie and Shorthouse, 2000).

Ivanova et al. (2003) have proposed a method to analyze heat capacity traces of lipid-peptide mixtures. The model presented by them strongly suggests that the peptide aggregation can be deduced from the analysis of calorimetric heat capacity data. Measuring heat capacity profiles of DMPC and DPPC lipid suspensions close to their gel/fluid transition containing 
alamethicin or melittin we found that the position of the main transition peak in heat capacity profiles are only very slightly shifted to lower temperatures with a small asymmetry of the peak shape at the low temperature wing. Larger amounts of peptides do not alter this behavior significantly. Applying the mentioned analysis (see chapter 1 on page 28 ) proposed by Ivanova et al. (2003) we can assume that for both alamethicin and melittin the lipid melting behavior of DMPC and DPPC membranes is very similar to that expected for a peptide, that aggregates in both gel and fluid phases, with a slight preference for the fluid phase.

Furthermore, in the $c_{P}$-profiles obtained for extruded DMPC lipid vesicle suspensions containing alamethicin we observed a splitting of the transition peak. Such splitting in heat capacity profiles was reported previously for pure DMPC vesicles (Heimburg, 1998). It is believed to be related to the changes of vesicle geometry in the lipid melting regime, by analogy to a transition between lipid vesicles and a bilayer network during lipid phase transition of DMPG dispersions detected by Schneider et al. (1999) in electron microscopy experiments. According to our measurements, the presence of alamethicin did not lead to any considerable changes in the splitting of the transition peak of DMPC vesicles, even in the case of high peptide concentrations. In $c_{P^{-}}$ profiles of DPPC suspensions with alamethicin we found that at $2 \mathrm{~mol} \%$ of alamethicin a small second peak appears at the high temperature wing of the main transition peak. It is more pronounced in the $c_{P}$-profile for $3 \mathrm{~mol} \%$ of peptide and almost disappears with further increasing of alamethicin content up to $4 \mathrm{~mol} \%$. Pure DPPC normally does not demonstrate any splitting of its phase transition peak. Hence, alamethicin molecules obviously induce structural transitions in the DPPC vesicles in the melting regime and this effect is concentration dependent.

In previous studies it was shown that the enthalpy change in lipid gelto-fluid phase transitions is proportional to changes in volume (Anthony et al., 1981; Heimburg, 1998; Ebel et al., 2001). Assuming such a proportional relationship between enthalpy and volume $\left(\Delta V^{\text {lipid }}(T)=\gamma \Delta H^{\text {lipid }}(T)\right.$, 
$\left.\gamma=7.8 \times 10^{-4} \mathrm{~cm}^{3} / \mathrm{J}^{3}\right)$, one can correctly predict the ultrasonic velocity profiles of lipid suspensions from the heat capacity. This was demonstrated in a number of studies for different lipid systems such as pure DMPC and DMPC-cholesterol mixtures (Halstenberg et al., 1998), various binary lipid mixtures (Schrader et al., 2002), even complex mixtures as the lung surfactant, which is composed of a variety of lipids as well as of several proteins (Ebel et al., 2001). Not only the absolute values but also the overall shape in the temperature dependence of the sound velocity were correctly predicted by the heat capacity data. For DPPC and DMPC lipid suspensions containing alamethicin we found that the velocity numbers measured in ultrasonic experiments can be perfectly fitted with velocity numbers recalculated from heat capacity profiles. Different peptide concentrations led to shape transformations of velocity number curves in a similar way than the changes in heat capacity profiles induced by alamethicin. With these results we demonstrated the deep linkage between the different thermodynamic response functions, such as heat capacity and adiabatic compressibility, to exist also for lipid-peptide mixtures.

Using Atomic Force Microscopy we were able to visualize directly alamethicin and melittin aggregation in phosphocholine lipid bilayers. In the case of melittin it was found that at its $1 \mathrm{~mol} \%$ concentration it aggregates forming transmembrane pores in both DPPC and DLPC lipid bilayers supported on mica. Since at room temperature DPPC is in gel phase and DLPC is in fluid phase, such pore formation by melittin in both membranes observed in AFM experiments confirms the predictions made from the analysis of its heat capacity profiles. However, it should be noted that in gel DPPC and fluid DLPC bilayers melittin formed pores in a different way. In DPPC bilayers melittin molecules aggregate forming elongated polyline defects, probably, following the high ordering of lipids in the gel phase, whereas in DLPC membranes the melittin pore formation process was found to result in a highly disordered and branched net of pores. For alamethicin-containing DPPC bilayers we recorded AFM images for its different concentrations. In height 
images of lipid membranes containing 1 mol \% of alamethicin we observed a formation of preferentially round shaped pores and a small number of elongated pores with characteristic smooth round kinks, whereas with the four times increased alamethicin content pores with a long, branched and irregular shape mostly develop. The sizes of alamethicin pores were measured to be in the range of $30-200 \mathrm{~nm}$ that is in agreement with a broad distribution of aggregation number, i.e., the number of peptides in the aggregate, reported from conductivity experiments (Sansom, 1991; Keller et al., 1993).

Another important result of our AFM studies of the peptide containing lipid bilayers is the existence of nanoscopic domains of lower height in close vicinity to the peptide induced pores. In AFM images of the gel DPPC bilayers with alamethicin or melittin we found local depressions around peptide pores, heights of which correspond to the thickness of DPPC membrane in fluid phase as found in literature (Heimburg, 1998). This led us to the conclusion that in a gel lipid membrane the aggregation of alamethicin and melittin triggers the surrounding lipids to melt. This melting is caused by a hydrophobic mismatch which dictates the hydrophobic part of the proteins or peptides for energetic reasons to be matched to the hydrophobic thickness of the lipid bilayer membrane in which they are embedded (Jensen and Mouritsen, 2004). In other words alamethicin molecules, for example, which have lengths of $\sim 3.5 \mathrm{~nm}$ (Fox and Richards, 1982), tend rather to be surrounded by a DPPC bilayer in fluid phase which has a thickness of $\sim 3.9 \mathrm{~nm}$ than by a DPPC bilayer in the gel phase with a thickness of $\sim 4.8 \mathrm{~nm}$ (Heimburg, 1998). Such an influence of peptide aggregates on the thermodynamic state of contacting lipids was also demonstrated in Monte Carlo simulations of peptide containing membranes performed by Ivanova et al. (2003). They have shown that the lipid fluctuations are higher around peptide aggregates embedded into the gel lipid matrix, which means a higher probability to find lipid molecules in a fluid state. Our AFM results can be regarded as an experimental confirmation of the theoretical findings. 
In the presented work we have attemptted to improve our understanding of the factors which lead to the formation of lipid membrane spatial heterogeneities, caused by mixtures of different lipids and/or transmembrane peptides. The demonstrated microscopic organization of lipid macrodomains in simple two-component lipid bilayers is of primary interest for structural biology where the origins and functions of lipid domains are currently intensively discussed. The phase behavior and structural domain formation approximates cell membrane behavior encountered in a native environment and points the way to an improved understanding of the physico-chemical properties of cell membranes and their associated physiology. In turn, directly observed and quantitatively analyzed peptide aggregation in phosphocholine membranes gives insights into the mechanisms behind protein-lipid interactions, the importance of which for the biological membrane functioning can hardly be overestimated. 


\section{Bibliography}

B. Alberts, D. Bray, J. Lewis, M. Raff, K. Roberts, and J. D. Watson. Molecular biology of the cell, chapter 10, pages 477-506. Garland Publishing, 1994.

F. H. Anthony, R. L. Biltonen, and E. Freire. Modification of a vibrating-tube density meter for precise temperature scanning. Analytical Biochemistry, 116 (1):161-167, Sept. 1981.

M. Argaman, R. Golan, N. Thomson, and H. Hansma. Phase imaging of moving DNA molecules and DNA molecules replicated in the atomic force microscope. Nucleic Acids Research, 25(21):4379-84, Nov 1997.

L. Bagatolli and E. Graton. Two-photon fluorescence microscopy observation of shape changes at the phase transition in phospholipid giant unilamellar vesicles. Biophysical Journal, 77:2090-2101, 1999.

B. Bechinger. Structure and functions of channel-forming peptides: magainins, cecropins, melittin and alamethicin. Journal of Membrane Biology, 156(3):197211, Apr 1997.

G. Beggerow. Landolt Börnstein IV: High-pressure properties of matter. Springer: Berlin, 1980.

J. M. Berg, J. L. Tymoczko, and L. Stryer. Biochemistry, chapter 12: Lipids and Cell Membranes., pages 319-344. W.H. Freeman and Company, fifth edition edition, 2002.

G. Binnig, C. Quate, and C. Gerber. Atomic force microscope. Physical Review Letters, 56(9):930-933, March 1986. 
D. Brown and E. London. Functions of lipid rafts in biological membranes. Annual Review of Cell and Developmental Biology, 14:111-36, 1998.

D. S. Cafiso. Alamethicin: a peptide model for voltage gating and proteinmembrane interactions. Annual Review of Biophysics and Biomolecular Structure, 23:141-65, 1994.

C. Cantor. Biophysical chemistry. Freeman and Company, New York, 1999.

W. Christie. High-perfomance of liquid cromotography and lipids. Pergamon Books, 1987.

M. Dathe and T. Wieprecht. Structural features of helical antimicrobial peptides: their potential to modulate activity on model membranes and biological cells. Biochimica et Biophysica Acta, 1462(1-2):71-87, Dec 1999.

C. E. Dempsey. The actions of melittin on membranes. Biochimica et Biophysica Acta (BBA) - Reviews on Biomembranes, 1031(2):143-161, 1990.

C. Dotti, R. Parton, and K. Simons. Polarized sorting of glypiated proteins in hipocampal neurons. Nature, 349:158-161, 1991.

W. Dowhan. Molecular basis for membrane phospholipid diversity: why are there so many lipids? Annual Review of Biochemistry, 66:199-232, 1997.

H. Duclohier and H. Wróblewski. Voltage-dependent pore formation and antimicrobial activity by alamethicin and analogues. The Journal of Membrane Biology, 184(1):1-12, Nov 2001.

F. Dumas, M. C. Lebrun, and J.-F. Tocanne. Is the protein/lipid hydrophobic matching principle relevant to membrane organization and functions? FEBS Letters, 458:271-277, 1999.

H. Ebel, P. Grabitz, and T. Heimburg. Enthalpy and volume changes in lipid membranes. i. the proportionality of heat and volume changes in the lipid melting transition and its implication for the elastic constants. The Journal of Physical Chemistry B, 105:7353-60, 2001. 
M. Edidin. Lipids on the frontier: a century of cell-membrane bilayers. Nature Reviews. Molecular Cell Biology, 4:414-418, May 2003.

F. Eggers and U. Kaatze. Broad-band ultrasonic measurement techniques for liquids. Measurement Science and Technology, 7:1-19, 1996.

G. Ehrenstein and H. Lecar. Electrically gated ionic channels in lipid bilayers. Quarterly Reviews of Biophysics, 10(1):1-34, Feb 1977.

J. F. Faucon, J. M. Bonmatin, J. Dufourcq, and E. J. Dufourc. Acyl chain length dependence in the stability of melittin-phosphatidylcholine complexes. A light scattering and 31P-NMR study. Biochimica et Biophysica Acta, 1234(2):235-43, Mar 1995.

R. O. Fox and F. M. Richards. A voltage gated ion channel inferred from the crystal structure of alamethicin at 1.5 å resolution. Nature, 300:325-330, 1982.

R. B. Gennis. Biomembranes: molecular structure and function. Springer Verlag, New York, 1989.

P. Grabitz, V. P. Ivanova, and T. Heimburg. Relaxation kinetics of lipid membranes and its relation to the heat capacity. Biophysical Journal, 82:299-309, 2002 .

M. Gribskov, L. Wesson, and D. Eisenberg. Melittin, the 2mlt protein. Structure available online from http://www.rcsb.org/pdb/, October 1990.

A. E. Hac, H. M. Seeger, M. Fidorra, and T. Heimburg. Diffusion in two-component lipid membranes - a fluorescence correlation spectroscopy and monte carlo simulation study. Biophysical Journal, 88:317-333, 2005.

S. Halstenberg, T. Heimburg, T. Hianik, U. Kaatze, and R. Krivanek. Cholesterolinduced variations in the volume and enthalpy fluctuations of lipid bilayers. Biophysical Journal, 75:264-271, 1998.

H. G. Hansma, K. J. Kim, D. E. Laney, R. A. Garcia, M. Argaman, M. J. Allen, and S. M. Parsons. Properties of biomolecules measured from atomic force microscope images: a review. Journal of Structural Biology, 119(2):99-108, Jul 1997. 
P. Hansma, J. Cleveland, M. Radmacher, D. Walters, P. Hillner, M. Bezanilla, M. Fritz, D. Vie, H. Hansma, C. Parter, J. Massie, L. Fukunaga, J. Gurley, and V. Elings. Tapping mode atomic force microscopy in liquids. Applied Physics Letters, 64(13):1738-1740, 1994.

T. Heimburg. Mechanical aspects of membrane thermodynamics. Estimation of the mechanical properties of lipid membranes close to the chain melting transition from calorimetry. Biochimica et Biophysica Acta, 1415:147-162, 1998.

T. Heimburg. A model for the lipid pretransition: coupling of ripple formation with the chain-melting transition. Biophysical Journal, 78(3):1154-1165, 2000.

T. Heimburg and R. L. Biltonen. A Monte Carlo simulation study of proteininduced heat capacity changes. Biophysical Journal, 70:84-96, 1996.

T. Heimburg and D. Marsh. Biological membranes: a molecular perspective from computation and experiment, chapter Thermodynamics of the interaction of proteins with lipid membranes, pages 405-462. Birkhäuser, Boston, 1996.

T. Hønger, K. Jørgensen, R. L. Biltonen, and O. G. Mouritsen. Systematic relationship between phospholipase A2 activity and dynamic lipid bilayer microheterogeneity. Biochemistry, 35(28):9003-6, Jul 1996.

V. P. Ivanova. Theoretical and experimental study of protein-lipid interactions. $\mathrm{PhD}$ thesis, University of Göttingen, 2000.

V. P. Ivanova and T. Heimburg. Histogram method to obtain heat capacities in lipid monolayers, curved bilayers, and membranes containing peptides. Physical Review E, 63:1914-25, 2001.

V. P. Ivanova, I. M. Makarov, T. E. Schäffer, and T. Heimburg. Analyzing heat capacity profiles of peptide-containing membranes: cluster formation of gramicidin A. Biophysical Journal, 84:2427-39, April 2003.

K. Jacobson and C. Dietrich. Looking at lipid rafts? Trends in Cell Biology, 9(3): 87-91, 1999. 
M. K. Jain and H. B. White. Long-range order in biomembranes. Advances in Lipid Research, 15:1-60, 1977.

M. J. Janiak, D. M. Small, and G. G. Shipley. Temperature and compositional dependence of the structure of hydrated dimyristoyl lecithin. The Journal of Biological Chemistry, 254(13):6068-78, Jul 1979.

M. Ø. Jensen and O. G. Mouritsen. Lipids do influence protein function - the hydrophobic matching hypothesis revisited. Biochimica et Biophysica Acta, 1666: 205-226, 2004.

T. Kaasgaard. Lipid bilayer investigations by atomic force microscopy. Domains, ripple phases, and interactions with enzymes, proteins, and peptides. $\mathrm{PhD}$ thesis, Technical University of Denmark, January 2003.

S. L. Keller, S. M. Bezrukov, S. M. Gruner, M. W. Tate, I. Vodyanoy, and V. A. Parsegian. Probability of alamethicin conductance states varies with nonlamellar tendency of bilayer phospholipids. Biophysical Journal, 65:23-27, 1993.

P. K. J. Kinnunen. On the molecular-level mechanisms of peripheral proteinmembrane interactions induced by lipids forming inverted non-lamellar phases. Chemistry and Physics of Lipids, 81(2):151-166, July 1996.

J. I. Kourie and A. A. Shorthouse. Properties of cytotoxic peptide-formed ion channels. American Journal of Physiology. Cell Physiology., 278(6):C1063-87, Jun 2000 .

T. Kurzchalia and R. Parton. Membrane microdomains and caveolae. Current Opinion in Cell Biology, 11(4):424-31, Aug 1999.

A. Kusumi and Y. Sako. Cell surface organization by the membrane skeleton. Current Opinion in Structural Biology, 8(4):566-74, Aug 1996.

K. Larsson. Lipids. Molecular organization, physical functions and technical applications, volume 5 of The Oily Press Lipid Library. The Oily Press, 1994.

C. Leidy, T. Kaasgaard, J. H. Crowe, O. G. Mouritsen, and K. Jørgensen. Ripples and the formation of anisotropic lipid domains: imaging two-component 
supported double bilayers by atomic force microscopy. Biophysical Journal, 83: 2625-2633, 2002.

D. R. Lide and H. P. R. Frederikse. Handbook of chemistry and physics. CRC Press: Boca Raton, FL, 77th edition, 1996.

R. C. MacDonald, R. I. MacDonald, B. P. M. Menco, K. Takeshita, N. K. Subbarao, and L. rong Hu. Small-volume extrusion apparatus for preparation of large, unilamellar vesicles. Biochimica et Biophysica Acta, 1061:297-303, 1991.

I. Makarov. Theoretical and experimental description of permeability of peptidecontaining membranes. PhD thesis, University of Göttingen, 2005.

L. Makowski and J. Li. Topics in Molecular and structural Biology: Biomembrane Structure and Function., chapter X-ray diffraction and electron microscope studies of the molecular structure of biological membranes., pages 43-166. Weinheim, 1984.

D. Marsh. Peptide models for membrane channels. The Biochemical Journal, 315 (2):345-361, Apr 1996.

S. Mitaku, T. Jippo, and R. Kataoka. Thermodynamic properties of the lipid bilayer transition. Pseudocritical phenomena. Biophysical Journal, 42(2):13744, May 1983.

V. Morris, A. Kirby, and A. Gunning. Atomic force microscopy for biologists. Imperial College Press, London, 2001.

M. R. Morrow, J. H. Davis, F. J. Sharom, and M. P. Lamb. Studies of the interaction of human erythrocyte band 3 with membrane lipids using deuterium nuclear magnetic resonance and differential scanning calorimetry. Biochimica et Biophysica Acta, 858:13-20, 1986.

J. Mou, J. Yang, and Z. Shao. Tris(hydroxymethyl)aminomethane (C4H11NO3) induced a ripple phase in supported unilamellar phospholipid bilayers. Biochemistry, 33(15):4439-43, Apr 1994. 
O. G. Mouritsen. Chemistry and physics of lipids, volume 57, pages 179-194. 1991a.

O. G. Mouritsen. Theoretical models of phospholipid phase transitions. Chemical physics letters, 57:179-194, 1991b.

O. G. Mouritsen and M. Bloom. Models of lipid-protein interactions in membranes. Annu Rev Biophys Biomol Struct, 22:145-71, 1993.

J. F. Nagle. Theory of the main lipid bilayer phase transition. Ann. Rev. Phys. Chem., 31:157-195, 1980.

L. K. Nielsen, A. Vishnyakov, K. Jørgensen, T. Bjørnholm, and O. G. Mouritsen. Nanometre-scale structure of fluid lipid membranes. Journal of Physics: Condensed Matter, 12(8A):A309-A314, 2000.

W. W. V. Osdol, R. L. Biltonen, and M. L. Johnson. Measuring the kinetics of membrane phase transitions. Journal of Biochemical and Biophysical Methods, 20(1):1-46, 1989.

G. K. H. Pang, K. Z. Baba-Kishi, and A. Patel. Topographic and phase-contrast imaging in atomic force microscopy. Ultramicroscopy, 81(2):35-40, Mar 2000.

V. V. Plotnikov, J. M. Brandts, L.-N. Lin, and J. F. Brandts. A new ultrasensitive scanning calorimeter. Analytical Biochemistry, 250(2):237-244, 1997.

T. Pott and E. J. Dufourc. Action of melittin on the DPPC-cholesterol liquidordered phase: a solid state 2H-and 31P-NMR study. Biophysical Journal, 68 (3):965-77, Mar 1995.

Y. Pouny, D. Rapaport, A. Mor, P. Nicolas, and Y. Shai. Interaction of antimicrobial dermaseptin and its fluorescently labeled analogues with phospholipid membranes. Biochemistry, 31(49):12416-23, Dec 1992.

E. J. Prenner, R. N. A. H. Lewis, L. H. Kondejewski, R. S. Hodges, and R. N. McElhaney. Differential scanning calorimetric study of the effect of the antimicrobial peptide gramicidin $\mathrm{S}$ on the thermotropic phase behavior of phosphatidylcholine, phosphatidylethanolamine and phosphatidylglycerol lipid bilayer membranes. Biochimica et Biophysica Acta, 1417:211-223, 1999. 
H. A. Rinia and B. de Kruiji. Imaging domains in model membranes with atomic force microscopy. FEBS Letters, 504:194-199, 2001.

H. A. Rinia, R. A. Kik, R. A. Demel, M. M. E. Snel, J. A. Killian, J. P. J. M. van der Eerden, and B. de Kruijff. Visualization of highly ordered striated domains induced by transmembrane peptides in supported phosphatidylcholine bilayers. Biochemistry, 39:5852-58, 2000.

D. Rugar and P. Hansma. Atomic force microscopy. Physics Today, 43:23-30, 1990.

E. Sackmann. Biological membranes, volume 5, chapter Physical basis for trigger processes and membrane structures, pages 105-143. Academic Press, London, 1984.

E. Sackmann. The seventh Datta Lecture. Membrane bending energy concept of vesicle- and cell-shapes and shape-transitions. FEBS Letters, 346(1):3-16, Jun 1994.

M. S. P. Sansom. The biophysics of peptide models of ion channels. Progress in Biophysics and Molecular Biology, 55:139-235, 1991.

B. B. Sauer, R. S. McLean, and R. R. Thomas. Tapping mode AFM studies of nano-phases on fluorine-containing polyester coatings and octadecyltrichlorosilane monolayers. Langmuir, 14(11):3041-3044, 1998.

M. F. Schneider, D. Marsh, W. Jahn, B. Kloesgen, and T. Heimburg. Network formation of lipid membranes: triggering structural transitions by chain melting. Proceedings of the National Academy of Sciences of the United States of America, 96(25):14312-7, Dec 1999.

W. Schrader, H. Ebel, P. Grabitz, E. Hanke, T. Heimburg, M. Hoeckel, M. Kahle, F. Wente, and U. Kaatze. Compressibility of lipid mixtures studied by calorimetry and ultrasonic velocity measurements. The Journal of Physical Chemistry $B, 106(25): 6581-6586,2002$.

H. Seeger, M. Fidorra, and T. Heimburg. Domain size and fluctuations at domain interfaces in lipid mixtures. Macromol. Symp., 219:85-96, 2005. 
Y. Shai. Mechanism of the binding, insertion and destabilization of phospholipid bilayer membranes by k-helical antimicrobial and cell non-selective membranelytic peptides. Biochimica et Biophysica Acta, 1462:55-70, 1999.

Z. Shao and J. Yang. Progress in high resolution atomic force microscopy in biology. Quarterly Reviews of Biophysics, 28(2):195-251, 1995.

K. Simons and E. Ikonen. Functional rafts in cell membranes. Nature, 387(6633): 569-72, Jun 1997.

K. Simons and G. van Meer. Lipid sorting in epithelial cells. Biochemistry, 27(17): 6197-202, Aug 1988.

S. Singer and G. Nicolson. The fluid mosaic model of the structure of cell membranes. Science, 175:720-731, 1972.

T. Stauffer and T. Meyer. Compartmentalized IgE receptor-mediated signal transduction in living cells. The Journal of Cell Biology, 139(6):1447-54, Dec 1997.

L. K. Tamm and H. M. McConnell. Supported phospholipid bilayers. Biophysical Journal, 47(1):105-13, Jan 1985.

B. G. Tenchov, H. Yao, and I. Hatta. Time-resolved x-ray diffraction and calorimetric studies at low scan rates: I. fully hydrated dipalmitoylphosphatidylcholine (dppc) and dppc/water/ethanol phases. Biophysical Journal, 56:757-768, 1989.

T. E. Thompson and T. W. Tillack. Organization of glycosphingolipids in bilayers and plasma membranes of mammalian cells. Annual review of biophysics and biophysical chemistry, 14:361-86, 1985.

F. Tokumasu, A. J. Jin, G. W. Feigenson, and J. A. Dvorak. Nanoscopic lipid domain dynamics revealed by atomic force microscopy. Biophysical Journal, 84: 2609-18, 2003.

W. W. van Osdol, M. L. Johnson, Q. Ye, and R. Biltonen. Relaxation dynamics of the gel to liquid-crystalline transition of phosphatidylcholine bilayers. Effects of chainlength and vesicle size. Biophysical Journal, 59:775-785, 1991. 
A. J. Verkleij, R. F. Zwaal, B. Roelofsen, P. Comfurius, D. Kastelijn, and L. L. van Deenen. The asymmetric distribution of phospholipids in the human red cell membrane. A combined study using phospholipases and freeze-etch electron microscopy. Biochimica et Biophysica Acta, 323(2):178-93, Oct 1973.

R. Wiesendanger. Scanning probe microscopy. Analytical methods. Springer, Berlin, 1998.

L. Yang and M. Glaser. Formation of membrane domains during the activation of protein kinase C. Biochemistry, 35(44):13966-74, Nov 1996.

A. Zemel, D. R. Fattal, and A. Ben-Shaul. Energetics and self-assembly of amphipathic peptide pore in lipid membranes. Biophysical Journal, 84:2242-55, 2003.

Q. Zhong, D. Inniss, K. Kjoller, and V. B. Elings. Fractured polymer/silica fiber surface studied by tapping mode atomic force microscopy. Surface Science, 290 (1-2):L688-L692, 1993. 


\section{List of Figures}

1.1 Schematic illustration of an animal cell . . . . . . . . . . . 9

1.2 Schematic illustration of the plasma membrane of a cell . . . . 10

1.3 Schematic illustration of a membrane raft . . . . . . . . . . 11

1.4 The example schema of a phospholipid . . . . . . . . . . . 12

1.5 Examples of lipid structures in water solutions . . . . . . . . 15

1.6 Lipid bilayer and lipid vesicle . . . . . . . . . . . . . . . 16

1.7 Scheme of the melting transition in a lipid bilayer . . . . . . . 17

1.8 Heat capacity profile of a DPPC aqueous dispersion. . . . . . 18

1.9 Confocal fluorescence microscopy image and Monte Carlo simulation snapshot of binary lipid mixtures . . . . . . . . . . . 19

1.10 Schematic illustration of hydrophobic matching between proteins and membrane thickness . . . . . . . . . . . . 21

1.11 A cartoon illustrating the "barrel-stave" and the "carpet" models suggested for membrane permeation . . . . . . . . 23

1.12 Atomic force micrographs of lipid membranes deposited on mica in the presence of gramicidin A . . . . . . . . 25

1.13 DPPC bilayers with 2 mol \% model peptides incorporated . . 26

1.14 AFM micrograph of a DPPC bilayer containing $\mathrm{C}_{14}$-peptide . 28

1.15 Calculated heat capacity profiles and Monte Carlo snapshots of peptide-containing lipid membranes: four limiting cases . . 29

1.16 MC snapshots, averaged MC snapshots, and the corresponding local fluctuations for a DPPC-gramicidin A system with 10 mol $\%$ peptide . . . . . . . . . . . . . . . . . 31 
2.1 The schematic illustration of AFM. . . . . . . . . . . 38

2.2 Interatomic force vs. distance curve. . . . . . . . . . . . . . . 40

2.3 A typical color scale used for topographic AFM-images. . . . . 41

2.4 Vibrating tip in the tapping mode . . . . . . . . . . . . . . . 42

2.5 Illustration of phase imaging . . . . . . . . . . . . . . . . 43

2.6 Principle scheme of a differential scanning calorimeter. . . . . 45

2.7 The cross section of a fixed-path cell . . . . . . . . . . . 48

2.8 Crystal structure of melittin and alamethicin molecules . . . . 50

2.9 Preparation of large unilamellar vesicles for calorimetry and ultrasonic experiments using the extrusion procedure and supported lipid bilayers for AFM-experiments using the direct fusion of small unilamellar vesicles on mica. . . . . . . . . . . . . 54

3.1 DPPC bilayers supported on mica . . . . . . . . . . . 58

3.2 DPPC bilayer in ripple phase . . . . . . . . . . . . . . 59

3.3 Tapping mode images of a DMPC membrane . . . . . . . . . . 60

3.4 Tapping mode images of a DLPC membrane . . . . . . . . . . 61

3.5 AFM image of DLPC:DPPC 33:67 membrane at room temperature . . . . . . . . . . . . . . . 63

3.6 Height and phase images of DLPC:DPPC 33:67 lipid bilayer . 64

3.7 Heat capacity profiles of DMPC and DPPC lipid vesicle suspensions with different alamethicin and melittin content. . . . 66

$3.8 c_{P}$-profiles and velocity number for DPPC extruded vesicle solutions containing alamethicin . . . . . . . . . . . 68

3.9 Density of a $2.5 \mathrm{mM}$ DMPC lipid suspension . . . . . . . . . . 69

3.10 Contact mode height images of a DPPC lipid membrane with 1 mol \% alamethicin . . . . . . . . . . . . . 71

3.11 Contact mode height images of a DPPC lipid membrane containing $4 \mathrm{~mol} \%$ alamethicin . . . . . . . . . . . . . 73

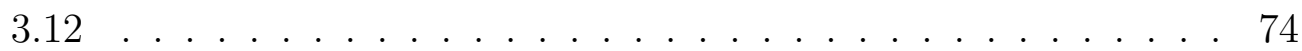

3.13 Height and phase images of a DPPC lipid membrane with 1 mol $\%$ alamethicin . . . . . . . . . . . . . . 75 
3.14 AFM images of DPPC and DLPC lipid membranes containing $1 \mathrm{~mol} \%$ of melittin . . . . . . . . . . . . . . . 77 


\section{Lebenslauf}

\section{Persönliche Daten}

Name: $\quad$ Vitaliy Oliynyk

Nationalität: ukrainisch

Geboren am 28.01.1980 in Nizhin, Ukraine.

\section{Ausbildung}

1986-1995 Grundschule in Nizhin, Ukraine.

1995-1997 Gymnasium in Nizhin, Ukraine.

1997-2002 Studium der Physik in der Fakultät für Physik

an der Taras Schewtschenko Nationaluniversität,

Kiew, Ukraine.

Fachgebiet: Medizinische Physik.

$06.2001 \quad$ Bakalaurusarbeit, ausgezeichnet.

Thema: "Niederigfrequenzfluktuationen im Herzgefäßsystem des Menschen".

Leiter: Prof. Yuriy Zabaschta.

$06.2002 \quad$ Magisterarbeit, ausgezeichnet.

Thema: "Verwendung vom ungleichartigen Diffusionsmodell bei der Beschrebung des Flickerlrms in den lebendigen Systemen". Leiter: Prof. Yuriy Zabaschta.

08.2002- Promotionsstudium in der Fakultät für Physik

07.2005 an der Georg-August-Iniversität zu Göttingen, Deutschland. Leiter: Prof. Thomas Heimburg/Dr. Udo Kaatze.

Forschungsgebiete: Rasterkraftmikroskopie an peptidhaltigen Lipidmembranen. 